\title{
Relativistic mean-field hadronic models under nuclear matter constraints
}

\author{
M. Dutra \\ Departamento de Física e Matemática-ICT, Universidade Federal Fluminense, 28895-532 Rio das Ostras, Rio de Janeiro, Brazil
}

\author{
O. Lourenço \\ Departamento de Ciências da Natureza, Matemática e Educação, CCA, Universidade Federal de São Carlos, \\ 13600-970 Araras, São Paulo, Brazil
}

S. S. Avancini

Depto de Física-CFM-Universidade Federal de Santa Catarina, CP 476, 88040-900 Florianópolis, Santa Catarina, Brazil

B. V. Carlson

Departamento de Física, Instituto Tecnológico de Aeronáutica, CTA, 12228-900 São José dos Campos, São Paulo, Brazil

A. Delfino

Departamento de Física-Universidade Federal Fluminense, Avenida Litorânea s/n, 24210-150 Boa Viagem, Niterói, Rio de Janeiro, Brazil

\author{
D. P. Menezes
}

Depto de Física-Universidade Federal de Santa Catarina, CP 476, 88040-900 Florianópolis, Santa Catarina, Brazil and Departamento de Física Aplicada, Universidad de Alicante, Apartado Correus 99, E-03080 Alicante, Spain

C. Providência

Centro de Física Computacional, Department of Physics, University of Coimbra, P-3004-516 Coimbra, Portugal

S. Typel

GSI Helmholtzzentrum für Schwerionenforschung GmbH, Theorie, Planckstrasse 1, D-64291 Darmstadt, Germany

J. R. Stone

Oxford Physics, University of Oxford, OX1 3PU Oxford, United Kingdom

and Department of Physics and Astronomy, University of Tennessee, Knoxville, Tennessee 37996, USA

(Received 14 May 2014; revised manuscript received 26 September 2014; published 10 November 2014)

Background: The microscopic composition and properties of infinite hadronic matter at a wide range of densities and temperatures have been subjects of intense investigation for decades. The equation of state (EoS) relating pressure, energy density, and temperature at a given particle number density is essential for modeling compact astrophysical objects such as neutron stars, core-collapse supernovae, and related phenomena, including the creation of chemical elements in the universe. The EoS depends not only on the particles present in the matter, but, more importantly, also on the forces acting among them. Because a realistic and quantitative description of infinite hadronic matter and nuclei from first principles in not available at present, a large variety of phenomenological models has been developed in the past several decades, but the scarcity of experimental and observational data does not allow a unique determination of the adjustable parameters.

Purpose: It is essential for further development of the field to determine the most realistic parameter sets and to use them consistently. Recently, a set of constraints on properties of nuclear matter was formed and the performance of 240 nonrelativistic Skyrme parametrizations was assessed [M. Dutra et al., Phys. Rev. C 85, 035201 (2012)] in describing nuclear matter up to about three times nuclear saturation density. In the present work we examine 263 relativistic-mean-field (RMF) models in a comparable approach. These models have been widely used because of several important aspects not always present in nonrelativistic models, such as intrinsic Lorentz covariance, automatic inclusion of spin, appropriate saturation mechanism for nuclear matter, causality, and, therefore, no problems related to superluminal speed of sound in medium.

Method: Three different sets of constraints related to symmetric nuclear matter, pure neutron matter, symmetry energy, and its derivatives were used. The first set (SET1) was the same as used in assessing the Skyrme parametrizations. The second and third sets (SET2a and SET2b) were more suitable for analysis of RMF and included, up-to-date theoretical, experimental and empirical information.

Results: The sets of updated constraints (SET2a and SET2b) differed somewhat in the level of restriction but still yielded only 4 and 3 approved RMF models, respectively. A similarly small number of approved Skyrme parametrizations were found in the previous study with Skyrme models. An interesting feature of our analysis 
has been that the results change dramatically if the constraint on the volume part of the isospin incompressibility $\left(K_{\tau, \mathrm{v}}\right)$ is eliminated. In this case, we have 35 approved models in SET2a and 30 in SET2b.

Conclusions: Our work provides a new insight into application of RMF models to properties of nuclear matter and brings into focus their problematic proliferation. The assessment performed in this work should be used in future applications of RMF models. Moreover, the most extensive set of refined constraints (including nuclear matter and finite-nuclei-related properties) should be used in future determinations of new parameter sets to provide models that can be used with more confidence in a wide range of applications. Pointing to reasons for the many failures, even of the frequently used models, should lead to their improvement and to the identification of possible missing physics not included in present energy density functionals.

DOI: 10.1103/PhysRevC.90.055203

PACS number(s): 21.30.Fe, 21.65.Cd, 21.65.Ef, 26.60.Kp

\section{INTRODUCTION}

The theoretical description of infinite nuclear matter and finite nuclear properties has been relying on models since the primordial developments of nuclear physics. Unfortunately, so far there are neither a specific nuclear physics theory nor enough adequate solutions for QCD, which is still in the early stages of lattice calculations. Many models have been developed since the beginning of the past century, from the famous semiempirical Bethe-Weizsäcker mass formula proposed in 1935 [1], to nonrelativistic Skyrme models [2,3] that first appeared around 1950, to relativistic quantum hadrodynamics (QHD) models [4] developed after 1974 , to which we devote our attention in the present work.

All relativistic models are written in terms of parameters that are fitted to reproduce either bulk nuclear matter or finite nuclei properties. This means that most models behave approximately the same as far as equations of state are concerned around saturation density and at zero temperature. Nevertheless, these very same models have been used to describe physics taking place at subsaturation densities, such as liquid-gas phase transitions, and also at very high densities, such as neutron-star matter. As a consequence, models that describe similar physics at saturation density yield very different results when used in the low- or high-density limits. The same holds true if finite systems are investigated. We devote the next section to the mean field-approximation usually employed when relativistic models are considered and to seven different types of parametrizations of QHD Walecka-type models. In these models, the baryons interact among each other by exchanging scalar-isoscalar $(\sigma)$ and vector-isoscalar $(\omega)$ mesons. For our analyses of nuclear matter properties, only nucleons are necessary as hadronic degrees of freedom. When the models are extended to describe stellar matter, hyperons are generally also included. More sophisticated versions include vector-isovector $(\rho)$ and vector-isoscalar $(\delta)$ mesons. The seven variations we treat next are (1) the original linear Walecka model, (2) the nonlinear Walecka model with $\sigma$ self-interacting mesons, (3) the nonlinear Walecka model with $\sigma$ and $\omega$ self-interacting mesons, (4) the nonlinear Walecka model with $\sigma$ and $\omega$ self-interacting mesons and possible mesonic cross terms, (5) models in which the parameters that couple the baryons with the mesons are density dependent, (6) point-coupling models, in which the baryons only interact with each other through effective pointlike interactions, without exchanging mesons, and (7) models with the inclusion of $\delta$ mesons. Details about the Lagrangian density, equations of motion, and equations of state for each of the seven model types are given in the next section.

In a previous work [5], 240 different Skyrme model parametrizations were confronted with experimentally and empirically derived constraints and only 16 of them were shown to satisfy all of the constraints. The authors argue that the production of new parameter sets with a limited range of application should not be encouraged. In the present work our aim is to obtain the physical properties related to the same derived constraints used in Ref. [5] with 263 relativistic models belonging to one of the seven classes mentioned above and check whether they satisfy these constraints.

A few words on our choice of constraints are now in order. We start with the isospin symmetric nuclear matter incompressibility (or compression modulus) $K_{0}$, the one most used to constrain mean-field models. The incompressibility values have been inferred from experiment and from theory. Experimentally, results coming from giant resonances, mainly isoscalar giant monopole resonance (GMR) [6] and isovector giant dipole resonance (GDR) [7] have been used as an important source of information. Theoretically, efforts to obtain values for the incompressibility started with the use of the Hartree-Fock plus random-phase approximation (RPA) [8] and continued to other calculations involving even more sophisticated treatments. The developments of these calculations can be tracked from many papers, but we mention specifically Refs. [9,10]. In the present paper, we follow the suggestions given in Refs. [11,12] for the value of $K_{0}=230 \pm 40 \mathrm{MeV}$.

Our second and third most important constraints are the symmetry energy $(J)$ and its slope $\left(L_{0}\right)$ at the saturation density. The density dependence of the symmetry energy carries information about the isospin dependence of nuclear forces and gives interesting hints on both finite nuclei and neutron-star properties. Experimental data for the symmetry energy come from various sources, namely heavy-ion collisions [13], pygmy dipole resonances [14,15], and isobaric analog states [16], besides GMR and GDR. In Ref. [17], the authors have shown that a direct correlation between the neutron skin thickness (controlled by the density dependence of the symmetry energy) and the neutron-star radii exists, such that models that yield smaller neutron skins in heavy nuclei generate smaller neutron-star radii. Recent reviews on this subject can be found in Refs. [18,19] and a comprehensive study of the imprint of the symmetry energy on the crust 
and strangeness content of neutron star can be seen in Ref. [20]. Moreover, in Refs. [21,22], a correlation between the values of the incompressibility and the symmetry energy was proposed based on the fact that the isoscalar giant monopole resonance (ISGMR) and isovector giant dipole resonance (IVGDR) of ${ }^{208} \mathrm{~Pb}$ were sensitive to both the incompressibility and the symmetry energy owing to its isospin asymmetry. Therefore, the author claims that the ISGMR data from a nucleus with a well-developed breathing mode but a small neutron-proton asymmetry such as ${ }^{90} \mathrm{Zr}$ should be used to fix the incompressibility at saturation instead of a nucleus with a non-negligible isospin asymmetry like ${ }^{208} \mathrm{~Pb}$. Once the incompressibility at saturation is fixed, the IVGDR ${ }^{208} \mathrm{~Pb}$ may be used to constrain the symmetry energy. Although not conclusive, there seems to exist a correlation among the values of $J, L_{0}$, and the curvature $\left(K_{\mathrm{sym}}^{0}\right)$ of the symmetry energy at the saturation density [23-25], and we tackle this point later.

Another constraint that we intend to use is the volume part of the isospin incompressibility, known as $K_{\tau, \mathrm{v}}$, which depends on several liquid drop model quantities. When it is extracted from a simple fitting to GMR data, it includes not only volume, but also surface contributions. According to isospin diffusion calculations [26], it should be $K_{\tau}=$ $-500 \pm 50 \mathrm{MeV}$. According to neutron skin thickness [27], $K_{\tau}=-500_{-100}^{+125} \mathrm{MeV}$, and according to GMR measured in Sn isotopes [28], $K_{\tau}=-550 \pm 100 \mathrm{MeV}$. To take into account all these uncertainties, we have chosen $K_{\tau}=-550 \pm 150 \mathrm{MeV}$.

The other constraints are obtained directly from the equation of state or are related to the constraints mentioned above and we add more comments after they are defined.

We note that we consider here only systems made of nucleons at zero temperature. Relativistic models are also used to describe systems at higher densities, including heavy baryons (hyperons) (see, e.g., [29]), and at finite temperatures, important in modeling of high-density matter in protoneutron stars and core-collapse supernovae, which we examine in a separate study.

Our paper is organized as follows. In Sec. II we present the basic equations that define the relativistic mean-field models we have chosen to analyze, such as the Lagrangian densities and their related equations of state. Our results and discussions, including the sets of constraints with which the models are confronted, are shown in Sec. III and we draw the conclusions in Sec. IV.

\section{RELATIVISTIC MEAN-FIELD MODELS AT ZERO TEMPERATURE}

Relativistic mean-field (RMF) models have been widely used to describe infinite nuclear matter (INM), finite nuclei, and stellar matter properties. The main representative of such kinds of models, the Walecka model, QHD, or linear Walecka model, as it is also known, proposed in 1974 [4], treats protons and neutrons as fundamental particles interacting with each other through the exchange of scalar and vector mesons. The $\sigma$ and $\omega$ fields represent, respectively, these mesons and mimic the attractive and repulsive parts of the nuclear interaction.

The two free parameters of the Walecka model, i.e., the couplings between the fields and the nucleons, are fitted to reproduce well-established properties of INM, namely, the binding energy $\left(E_{0} \sim 16 \mathrm{MeV}\right)$ and the saturation density $\left(\rho_{0} \sim 0.15 \mathrm{fm}^{-3}\right)$. However, it does not give reasonable values for the incompressibility $\left(K_{0}\right)$, and nucleon effective mass $\left(M^{*}\right)$, both related to symmetric nuclear matter (SNM). This problem was circumvented by Boguta and Bodmer [30], who added to the Walecka model cubic and quartic self-interactions in the scalar field $\sigma$, introducing, consequently, two more free parameters, which are fitted so as to fix the values of $K_{0}$ and $M^{*}$. In the same way, more terms can be added to the BogutaBodmer model to make it compatible with other observables, such as those related to finite nuclei. Actually, many RMF models and parametrizations have been constructed following this method. In our work, we take into account a more general nonlinear finite-range RMF model, by considering it to be represented by the Lagrangian density,

$$
\mathcal{L}_{\mathrm{NL}}=\mathcal{L}_{\mathrm{nm}}+\mathcal{L}_{\sigma}+\mathcal{L}_{\omega}+\mathcal{L}_{\rho}+\mathcal{L}_{\delta}+\mathcal{L}_{\sigma \omega \rho}
$$

where

$$
\begin{aligned}
\mathcal{L}_{\mathrm{nm}}= & \bar{\psi}\left(i \gamma^{\mu} \partial_{\mu}-M\right) \psi+g_{\sigma} \sigma \bar{\psi} \psi-g_{\omega} \bar{\psi} \gamma^{\mu} \omega_{\mu} \psi \\
& -\frac{g_{\rho}}{2} \bar{\psi} \gamma^{\mu} \vec{\rho}_{\mu} \vec{\tau} \psi+g_{\delta} \bar{\psi} \vec{\delta} \vec{\tau} \psi \\
\mathcal{L}_{\sigma}= & \frac{1}{2}\left(\partial^{\mu} \sigma \partial_{\mu} \sigma-m_{\sigma}^{2} \sigma^{2}\right)-\frac{A}{3} \sigma^{3}-\frac{B}{4} \sigma^{4}, \\
\mathcal{L}_{\omega}= & -\frac{1}{4} F^{\mu \nu} F_{\mu \nu}+\frac{1}{2} m_{\omega}^{2} \omega_{\mu} \omega^{\mu}+\frac{C}{4}\left(g_{\omega}^{2} \omega_{\mu} \omega^{\mu}\right)^{2}, \\
\mathcal{L}_{\rho}= & -\frac{1}{4} \vec{B}^{\mu \nu} \vec{B}_{\mu \nu}+\frac{1}{2} m_{\rho}^{2} \vec{\rho}_{\mu} \vec{\rho}^{\mu} \\
\mathcal{L}_{\delta}= & \frac{1}{2}\left(\partial^{\mu} \vec{\delta} \partial_{\mu} \vec{\delta}-m_{\delta}^{2} \vec{\delta}^{2}\right),
\end{aligned}
$$

and

$$
\begin{aligned}
\mathcal{L}_{\sigma \omega \rho}= & g_{\sigma} g_{\omega}^{2} \sigma \omega_{\mu} \omega^{\mu}\left(\alpha_{1}+\frac{1}{2} \alpha_{1}^{\prime} g_{\sigma} \sigma\right) \\
& +g_{\sigma} g_{\rho}^{2} \sigma \vec{\rho}_{\mu} \vec{\rho}^{\mu}\left(\alpha_{2}+\frac{1}{2} \alpha_{2}^{\prime} g_{\sigma} \sigma\right) \\
& +\frac{1}{2} \alpha_{3}^{\prime} g_{\omega}^{2} g_{\rho}^{2} \omega_{\mu} \omega^{\mu} \vec{\rho}_{\mu} \vec{\rho}^{\mu} .
\end{aligned}
$$

In this Lagrangian density, $\mathcal{L}_{\mathrm{nm}}$ stands for the kinetic part of the nucleons added to the terms representing the interaction between the nucleons and mesons $\sigma, \delta, \omega$, and $\rho$. The term $\mathcal{L}_{j}$ represents the free and self-interacting terms of the meson $j$, for $j=\sigma, \delta, \omega$, and $\rho$. The last term, $\mathcal{L}_{\sigma \omega \rho}$, takes into account crossed interactions between the meson fields. The antisymmetric field tensors $F_{\mu \nu}$ and $\vec{B}_{\mu \nu}$ are given by $F_{\mu \nu}=\partial_{\nu} \omega_{\mu}-\partial_{\mu} \omega_{v}$ and $\vec{B}_{\mu \nu}=\partial_{\nu} \vec{\rho}_{\mu}-\partial_{\mu} \vec{\rho}_{\nu}-g_{\rho}\left(\vec{\rho}_{\mu} \times \vec{\rho}_{\nu}\right)$. The nucleon mass is $M$ and the meson masses are $m_{j}$.

The use of the mean-field approximation, in which the meson fields are treated as classical fields as

$$
\begin{aligned}
\sigma & \rightarrow\left\langle\sigma \equiv \sigma, \quad \omega_{\mu} \rightarrow\left\langle\omega_{\mu}\right\rangle \equiv \omega_{0},\right. \\
\vec{\rho}_{\mu} & \rightarrow\left\langle\vec{\rho}_{\mu}\right\rangle \equiv \bar{\rho}_{0(3)}, \quad \text { and } \quad \vec{\delta} \rightarrow\langle\vec{\delta}\rangle \equiv \delta_{(3)},
\end{aligned}
$$

together with the Euler-Lagrange equations, leads to the following field equations:

$$
\begin{aligned}
m_{\sigma}^{2} \sigma= & g_{\sigma} \rho_{s}-A \sigma^{2}-B \sigma^{3}+g_{\sigma} g_{\omega}^{2} \omega_{0}^{2}\left(\alpha_{1}+\alpha_{1}^{\prime} g_{\sigma} \sigma\right) \\
& +g_{\sigma} g_{\rho}^{2} \bar{\rho}_{0(3)}^{2}\left(\alpha_{2}+\alpha_{2}^{\prime} g_{\sigma} \sigma\right),
\end{aligned}
$$




$$
\begin{aligned}
m_{\omega}^{2} \omega_{0}= & g_{\omega} \rho-C g_{\omega}\left(g_{\omega} \omega_{0}\right)^{3}-g_{\sigma} g_{\omega}^{2} \sigma \omega_{0}\left(2 \alpha_{1}+\alpha_{1}^{\prime} g_{\sigma} \sigma\right) \\
& -\alpha_{3}^{\prime} g_{\omega}^{2} g_{\rho}^{2} \bar{\rho}_{0(3)}^{2} \omega_{0} \\
m_{\rho}^{2} \bar{\rho}_{0(3)}= & \frac{g_{\rho}}{2} \rho_{3}-g_{\sigma} g_{\rho}^{2} \sigma \bar{\rho}_{0(3)}\left(2 \alpha_{2}+\alpha_{2}^{\prime} g_{\sigma} \sigma\right) \\
& -\alpha_{3}^{\prime} g_{\omega}^{2} g_{\rho}^{2} \bar{\rho}_{0(3)} \omega_{0}^{2} \\
m_{\delta}^{2} \delta_{(3)}= & g_{\delta} \rho_{s 3} \\
{\left[\gamma ^ { \mu } \left(i \partial_{\mu}-\right.\right.} & \left.\left.V_{\tau}\right)-\left(M+S_{\tau}\right)\right] \psi=0 .
\end{aligned}
$$

Owing to the translational invariance and rotational symmetry of INM, only the zero components of the four-vector fields are nonvanishing. Also considering rotational invariance around the third axis in isospin space, we only deal with the third components of the isospin space vectors $\vec{\rho}_{\mu}$ and $\vec{\delta}$.

The scalar and vector densities are given by

$$
\begin{aligned}
\rho_{s} & =\langle\bar{\psi} \psi\rangle=\rho_{s p}+\rho_{s_{n}}, \\
\rho_{s 3} & =\left\langle\bar{\psi} \tau_{3} \psi\right\rangle=\rho_{s_{p}}-\rho_{s_{n}}, \\
\rho & =\left\langle\bar{\psi} \gamma^{0} \psi\right\rangle=\rho_{p}+\rho_{n}, \\
\rho_{3} & =\left\langle\bar{\psi} \gamma^{0} \tau_{3} \psi\right\rangle=\rho_{p}-\rho_{n}=(2 y-1) \rho,
\end{aligned}
$$

with

$$
\begin{aligned}
\rho_{s p, n} & =\frac{\gamma M_{p, n}^{*}}{2 \pi^{2}} \int_{0}^{k_{F p, n}} \frac{k^{2} d k}{\sqrt{k^{2}+M_{p, n}^{* 2}}} \\
& =\frac{\gamma\left(M_{p, n}^{*}\right)^{3} q^{2}}{2 \pi^{2}} \int_{0}^{1} \frac{\xi^{2} d \xi}{\sqrt{\xi^{2}+1 / q^{2}}} \\
& =\frac{\gamma\left(M_{p, n}^{*}\right)^{3}}{4 \pi^{2}}\left[q \sqrt{1+q^{2}}-\ln \left(q+\sqrt{1+q^{2}}\right)\right],
\end{aligned}
$$

and

$$
\rho_{p, n}=\frac{\gamma}{2 \pi^{2}} \int_{0}^{k_{F p, n}} k^{2} d k=\frac{\gamma}{6 \pi^{2}} k_{F p, n}^{3},
$$

for $\xi=k / k_{F p, n}$ and $q=k_{F p, n} / M_{p, n}^{*}$ with the indices $p, n$ standing for protons and neutrons, respectively. The degeneracy factor is $\gamma=2$ for asymmetric matter, and the proton fraction is defined as $y=\rho_{p} / \rho$. The quantity $k_{F p, n}$ is the Fermi momentum in the units in which $\hbar=c=1$.

From the Dirac equation (13), we recognize the vector and scalar potentials written as

$$
\begin{aligned}
V_{\tau \mathrm{NL}} & =g_{\omega} \omega_{0}+\frac{g_{\rho}}{2} \bar{\rho}_{0(3)} \tau_{3} \quad \text { and } \\
S_{\tau \mathrm{NL}} & =-g_{\sigma} \sigma-g_{\delta} \delta_{(3)} \tau_{3},
\end{aligned}
$$

with $\tau_{3}=1$ and -1 for protons and neutrons, respectively. We can also define the effective nucleon mass as $M_{\tau}^{*}=M+$ $S_{\tau} \mathrm{NL}$, leading to

$$
\begin{aligned}
& M_{p}^{*}=M-g_{\sigma} \sigma-g_{\delta} \delta_{(3)} \quad \text { and } \\
& M_{n}^{*}=M-g_{\sigma} \sigma+g_{\delta} \delta_{(3)} .
\end{aligned}
$$

Note the effect of the meson $\delta$, which splits the effective masses $M_{p}^{*}$ and $M_{n}^{*}$. For SNM $\delta_{(3)}$ vanishes, because $\rho_{s p}=\rho_{s n}$, and consequently, $M_{p}^{*}=M_{n}^{*}=M^{*}=M-g_{\sigma} \sigma$.

From the energy-momentum tensor $T^{\mu \nu}$, calculated through the Lagrangian density in Eq. (1), it is possible to obtain the energy density and the pressure of the asymmetric system, because $\mathcal{E}=\left\langle T_{00}\right\rangle$ and $P=\left\langle T_{i i}\right\rangle / 3$. These quantities are given as

$$
\begin{aligned}
\mathcal{E}_{\mathrm{NL}}= & \frac{1}{2} m_{\sigma}^{2} \sigma^{2}+\frac{A}{3} \sigma^{3}+\frac{B}{4} \sigma^{4}-\frac{1}{2} m_{\omega}^{2} \omega_{0}^{2}-\frac{C}{4}\left(g_{\omega}^{2} \omega_{0}^{2}\right)^{2}-\frac{1}{2} m_{\rho}^{2} \bar{\rho}_{0(3)}^{2}+g_{\omega} \omega_{0} \rho+\frac{g_{\rho}}{2} \bar{\rho}_{0(3)} \rho_{3}+\frac{1}{2} m_{\delta}^{2} \delta_{(3)}^{2} \\
& -g_{\sigma} g_{\omega}^{2} \sigma \omega_{0}^{2}\left(\alpha_{1}+\frac{1}{2} \alpha_{1}^{\prime} g_{\sigma} \sigma\right)-g_{\sigma} g_{\rho}^{2} \sigma \bar{\rho}_{0(3)}^{2}\left(\alpha_{2}+\frac{1}{2} \alpha_{2}^{\prime} g_{\sigma} \sigma\right)-\frac{1}{2} \alpha_{3}^{\prime} g_{\omega}^{2} g_{\rho}^{2} \omega_{0}^{2} \bar{\rho}_{0(3)}^{2}+\mathcal{E}_{\text {kin }}^{p}+\mathcal{E}_{\text {kin }}^{n}
\end{aligned}
$$

where

$$
\begin{aligned}
\mathcal{E}_{\mathrm{kin}}^{p, n} & =\frac{\gamma}{2 \pi^{2}} \int_{0}^{k_{F p, n}} k^{2}\left(k^{2}+M_{p, n}^{* 2}\right)^{1 / 2} d k=\frac{\gamma k_{F p, n}^{4}}{2 \pi^{2}} \int_{0}^{1} \xi^{2}\left(\xi^{2}+z^{2}\right)^{1 / 2} d \xi \\
& =\frac{\gamma k_{F p, n}^{4}}{2 \pi^{2}}\left[\left(1+\frac{z^{2}}{2}\right) \frac{\sqrt{1+z^{2}}}{4}-\frac{z^{4}}{8} \ln \left(\frac{1+\sqrt{1+z^{2}}}{z}\right)\right]=\frac{3}{4} E_{F p, n} \rho_{p, n}+\frac{1}{4} M_{p, n}^{*} \rho_{s p, n},
\end{aligned}
$$

and

$$
\begin{aligned}
P_{\mathrm{NL}}= & -\frac{1}{2} m_{\sigma}^{2} \sigma^{2}-\frac{A}{3} \sigma^{3}-\frac{B}{4} \sigma^{4}+\frac{1}{2} m_{\omega}^{2} \omega_{0}^{2}+\frac{C}{4}\left(g_{\omega}^{2} \omega_{0}^{2}\right)^{2}+\frac{1}{2} m_{\rho}^{2} \bar{\rho}_{0(3)}^{2}+\frac{1}{2} \alpha_{3}^{\prime} g_{\omega}^{2} g_{\rho}^{2} \omega_{0}^{2} \bar{\rho}_{0(3)}^{2} \\
& -\frac{1}{2} m_{\delta}^{2} \delta_{(3)}^{2}+g_{\sigma} g_{\omega}^{2} \sigma \omega_{0}^{2}\left(\alpha_{1}+\frac{1}{2} \alpha_{1}^{\prime} g_{\sigma} \sigma\right)+g_{\sigma} g_{\rho}^{2} \sigma \bar{\rho}_{0(3)}^{2}\left(\alpha_{2}+\frac{1}{2} \alpha_{2}^{\prime} g_{\sigma} \sigma\right)+P_{\mathrm{kin}}^{p}+P_{\mathrm{kin}}^{n},
\end{aligned}
$$

with

$$
\begin{aligned}
P_{\text {kin }}^{p, n} & =\frac{\gamma}{6 \pi^{2}} \int_{0}^{k_{F p, n}} \frac{k^{4} d k}{\left(k^{2}+M_{p, n}^{* 2}\right)^{1 / 2}}=\frac{\gamma k_{F p, n}^{4}}{6 \pi^{2}} \int_{0}^{1} \frac{\xi^{4} d \xi}{\left(\xi^{2}+z^{2}\right)^{1 / 2}} \\
& =\frac{\gamma k_{F p, n}^{4}}{6 \pi^{2}}\left[\left(1-\frac{3 z^{2}}{2}\right) \frac{\sqrt{1+z^{2}}}{4}+\frac{3 z^{4}}{8} \ln \left(\frac{1+\sqrt{1+z^{2}}}{z}\right)\right]=\frac{1}{4} E_{F p, n} \rho_{p, n}-\frac{1}{4} M_{p, n}^{*} \rho_{s p, n},
\end{aligned}
$$


and

$$
E_{F p, n}=\sqrt{k_{F p, n}^{2}+\left(M_{p, n}^{*}\right)^{2}}
$$

In the above equations, the parameter $z$ is defined as $z=M_{p, n}^{*} / k_{F p, n}$.

To better identify the parametrizations related to the model described in Eq. (1), we define here four different types of parametrizations.

(i) Type 1 (linear finite range models): Models in which $A=B=C=\alpha_{1}=\alpha_{2}=\alpha_{1}^{\prime}=\alpha_{2}^{\prime}=\alpha_{3}^{\prime}=g_{\delta}=0$. This is the case of the linear Walecka model. Different parametrizations correspond to different values of the pair $\left(\rho_{0}, E_{0}\right)$.

(ii) Type $2\left(\sigma^{3}+\sigma^{4}\right.$ models $)$ : Models in which $C=\alpha_{1}=\alpha_{2}=\alpha_{1}^{\prime}=\alpha_{2}^{\prime}=\alpha_{3}^{\prime}=g_{\delta}=0$. This type corresponds to parametrizations related to the Boguta-Bodmer model.

(iii) Type $3\left(\sigma^{3}+\sigma^{4}+\omega_{0}^{4}\right.$ models): Models in which $\alpha_{1}=\alpha_{2}=\alpha_{1}^{\prime}=\alpha_{2}^{\prime}=\alpha_{3}^{\prime}=g_{\delta}=0$. These parametrizations include a quartic self-interaction in the $\omega$ field.

(iv) Type $4\left(\sigma^{3}+\sigma^{4}+\omega_{0}^{4}+\right.$ cross terms models): Models in which $g_{\delta}=0$ and at least one of the coupling constants, $\alpha_{1}, \alpha_{2}$, $\alpha_{1}^{\prime}, \alpha_{2}^{\prime}$, or $\alpha_{3}^{\prime}$ is different from zero.

Another widely used approach in QHD is that in which the couplings between nucleons and mesons are sensitive to the nuclear medium. Specifically, the RMF model proposed in Ref. [31] allows density dependence in the aforementioned couplings by making

$$
g_{\sigma} \rightarrow \Gamma_{\sigma}(\rho), \quad g_{\omega} \rightarrow \Gamma_{\omega}(\rho), \quad g_{\rho} \rightarrow \Gamma_{\rho}(\rho), \quad \text { and } \quad g_{\delta} \rightarrow \Gamma_{\delta}(\rho) .
$$

Its Lagrangian density is given by

$$
\begin{aligned}
\mathcal{L}_{\mathrm{DD}}= & \bar{\psi}\left(i \gamma^{\mu} \partial_{\mu}-M\right) \psi+\Gamma_{\sigma}(\rho) \sigma \bar{\psi} \psi-\Gamma_{\omega}(\rho) \bar{\psi} \gamma^{\mu} \omega_{\mu} \psi-\frac{\Gamma_{\rho}(\rho)}{2} \bar{\psi} \gamma^{\mu} \vec{\rho}_{\mu} \vec{\tau} \psi+\Gamma_{\delta}(\rho) \bar{\psi} \vec{\delta} \vec{\tau} \psi \\
& +\frac{1}{2}\left(\partial^{\mu} \sigma \partial_{\mu} \sigma-m_{\sigma}^{2} \sigma^{2}\right)-\frac{1}{4} F^{\mu \nu} F_{\mu \nu}+\frac{1}{2} m_{\omega}^{2} \omega_{\mu} \omega^{\mu}-\frac{1}{4} \vec{B}^{\mu \nu} \vec{B}_{\mu \nu}+\frac{1}{2} m_{\rho}^{2} \vec{\rho}_{\mu} \vec{\rho}^{\mu}+\frac{1}{2}\left(\partial^{\mu} \vec{\delta} \partial_{\mu} \vec{\delta}-m_{\delta}^{2} \vec{\delta}^{2}\right),
\end{aligned}
$$

where

$$
\begin{aligned}
\Gamma_{i}(\rho) & =\Gamma_{i}\left(\rho_{0}\right) f_{i}(x), \quad \text { with } \\
f_{i}(x) & =a_{i} \frac{1+b_{i}\left(x+d_{i}\right)^{2}}{1+c_{i}\left(x+d_{i}\right)^{2}},
\end{aligned}
$$

for $i=\sigma, \omega$, and

$$
\Gamma_{\rho}(\rho)=\Gamma_{\rho}\left(\rho_{0}\right) e^{-a(x-1)}, \quad \text { with } \quad x=\rho / \rho_{0} .
$$

Some density-dependent parametrizations have couplings different from those of the above equations. In particular, the GDFM model [32] presents a form for its couplings,

$$
\Gamma_{i}(\rho)=a_{i}+\left(b_{i}+d_{i} x^{3}\right) e^{-c_{i} x},
$$

for $i=\sigma, \omega, \rho, \delta$. A correction to the coupling parameter for the meson $\omega$ is also taken into account:

$$
\Gamma_{\text {cor }}(\rho)=\Gamma_{\omega}(\rho)-a_{\text {cor }} e^{-\left(\frac{\rho-\rho_{0}}{b_{\text {cor }}}\right)^{2}} .
$$

The DDH $\delta$ parametrization has the same coupling parameters as in Eq. (28) for the mesons $\sigma$ and $\omega$, but functions $f_{i}(x)$ given by [33]

$$
f_{i}(x)=a_{i} e^{-b_{i}(x-1)}-c_{i}\left(x-d_{i}\right),
$$

for $i=\rho, \delta$.

By applying the mean-field approximation and the EulerLagrange equations, we find the same field equations as in Eqs. (9)-(13), taking into account Eq. (26) and $A=B=C=$ $\alpha_{1}=\alpha_{2}=\alpha_{1}^{\prime}=\alpha_{2}^{\prime}=\alpha_{3}^{\prime}=0$. The scalar and vector densities are defined as in the previous nonlinear RMF model. The proton and neutron effective masses, $M_{p}^{*}$ and $M_{n}^{*}$, and the scalar potential are also defined as in Eqs. (20) and (19), respectively, observing the generalizations in Eq. (26). The same does not occur for the vector potential that now reads

$$
V_{\tau} \mathrm{DD}=\Gamma_{\omega}(\rho) \omega_{0}+\frac{\Gamma_{\rho}(\rho)}{2} \bar{\rho}_{0(3)} \tau_{3}+\Sigma_{R}(\rho)
$$

with

$$
\Sigma_{R}(\rho)=\frac{\partial \Gamma_{\omega}}{\partial \rho} \omega_{0} \rho+\frac{1}{2} \frac{\partial \Gamma_{\rho}}{\partial \rho} \bar{\rho}_{0(3)} \rho_{3}-\frac{\partial \Gamma_{\sigma}}{\partial \rho} \sigma \rho_{s}-\frac{\partial \Gamma_{\delta}}{\partial \rho} \delta_{(3)} \rho_{s 3}
$$

being the rearrangement term.

The energy density and pressure are given, respectively, by

$$
\begin{aligned}
\mathcal{E}_{\mathrm{DD}}= & \frac{1}{2} m_{\sigma}^{2} \sigma^{2}-\frac{1}{2} m_{\omega}^{2} \omega_{0}^{2}-\frac{1}{2} m_{\rho}^{2} \bar{\rho}_{0(3)}^{2}+\frac{1}{2} m_{\delta}^{2} \delta_{(3)}^{2} \\
& +\Gamma_{\omega}(\rho) \omega_{0} \rho+\frac{\Gamma_{\rho}(\rho)}{2} \bar{\rho}_{0(3)} \rho_{3}+\mathcal{E}_{\mathrm{kin}}^{p}+\mathcal{E}_{\mathrm{kin}}^{n}, \quad \text { and }
\end{aligned}
$$

$$
\begin{aligned}
P_{\mathrm{DD}}= & \rho \Sigma_{R}(\rho)-\frac{1}{2} m_{\sigma}^{2} \sigma^{2}+\frac{1}{2} m_{\omega}^{2} \omega_{0}^{2}+\frac{1}{2} m_{\rho}^{2} \bar{\rho}_{0(3)}^{2} \\
& -\frac{1}{2} m_{\delta}^{2} \delta_{(3)}^{2}+P_{\mathrm{kin}}^{p}+P_{\mathrm{kin}}^{n} .
\end{aligned}
$$

We also define here the fifth type of parametrization analyzed in our work.

(v) Type 5 (density-dependent models): Parametrizations obtained from Eq. (27) in which $\Gamma_{\delta}=0$.

Another class of RMF models is the nonlinear pointcoupling (NLPC) model [34]. In this theory, nucleons interact with each other only through effective pointlike interactions, without exchanging mesons. It can be easily proved that the 
linear version of the PC model results in the same equations of state as the linear Walecka model [35]. The same does not hold for NLPC models with cubic and quartic interactions and finite-range RMF models of type 2; besides, both versions can describe equally well INM [36]. Here, we treat a general type of NLPC model, described by the following Lagrangian density:

$$
\begin{aligned}
\mathcal{L}_{\mathrm{NLPC}}= & \bar{\psi}\left(i \gamma^{\mu} \partial_{\mu}-M\right) \psi-\frac{\alpha_{s}}{2}(\bar{\psi} \psi)^{2}-\frac{\beta_{s}}{3}(\bar{\psi} \psi)^{3} \\
& -\frac{\gamma_{s}}{4}(\bar{\psi} \psi)^{4}-\frac{\alpha_{\mathrm{V}}}{2}\left(\bar{\psi} \gamma^{\mu} \psi\right)^{2}-\frac{\gamma_{\mathrm{V}}}{4}\left(\bar{\psi} \gamma^{\mu} \psi\right)^{4} \\
& -\frac{\alpha_{\mathrm{TV}}}{2}\left(\bar{\psi} \gamma^{\mu} \vec{\tau} \psi\right)^{2}-\frac{\gamma_{\mathrm{TV}}}{4}\left(\bar{\psi} \gamma^{\mu} \vec{\tau} \psi\right)^{4}-\frac{\alpha_{\mathrm{TS}}}{2}(\bar{\psi} \vec{\tau} \psi)^{2} \\
& +\left[\eta_{1}+\eta_{2}(\bar{\psi} \psi)\right](\bar{\psi} \psi)\left(\bar{\psi} \gamma^{\mu} \psi\right)^{2} \\
& -\eta_{3}(\bar{\psi} \psi)\left(\bar{\psi} \gamma^{\mu} \vec{\tau} \psi\right)^{2} .
\end{aligned}
$$

The mean-field approximation gives rise to the Dirac equation given in Eq. (13), with the vector and scalar potentials modified, respectively, as

$$
\begin{aligned}
V_{\tau \mathrm{NLPC}}= & \alpha_{\mathrm{V}} \rho+\alpha_{\mathrm{TV}} \rho_{3} \tau_{3}+\gamma_{\mathrm{V}} \rho^{3}+\gamma_{\mathrm{TV}} \rho_{3}^{3} \tau_{3} \\
& +2\left(\eta_{1}+\eta_{2} \rho_{s}\right) \rho_{s} \rho+2 \eta_{3} \rho_{s} \rho_{3} \tau_{3}, \\
S_{\tau \mathrm{NLPC}}= & \alpha_{s} \rho_{s}+\beta_{s} \rho_{s}^{2}+\gamma_{s} \rho_{s}^{3}+\eta_{1} \rho^{2}+2 \eta_{2} \rho_{s} \rho^{2} \\
& +\eta_{3} \rho_{3}^{2}+\alpha_{\mathrm{TS}} \rho_{s} \tau_{3},
\end{aligned}
$$

with $\rho_{s}, \rho_{s 3}, \rho$, and $\rho_{3}$ defined as in Eqs. (14)-(17). The proton and neutron effective masses read

$$
\begin{aligned}
M_{p}^{*}= & M+\alpha_{s} \rho_{s}+\beta_{s} \rho_{s}^{2}+\gamma_{s} \rho_{s}^{3}+\eta_{1} \rho^{2}+2 \eta_{2} \rho_{s} \rho^{2} \\
& +\eta_{3} \rho_{3}^{2}+\alpha_{\mathrm{TS}} \rho_{s}, \\
M_{n}^{*}= & M+\alpha_{s} \rho_{s}+\beta_{s} \rho_{s}^{2}+\gamma_{s} \rho_{s}^{3}+\eta_{1} \rho^{2}+2 \eta_{2} \rho_{s} \rho^{2} \\
& +\eta_{3} \rho_{3}^{2}-\alpha_{\mathrm{TS}} \rho_{s} .
\end{aligned}
$$

Notice that the interaction whose magnitude is given by $\alpha_{\mathrm{TS}}$ plays the same role as the meson $\delta$ in the finite-range RMF models, namely, the splitting of proton and neutron effective masses.

Finally, we obtain the energy density and the pressure for the NLPC model as

$$
\begin{aligned}
\mathcal{E}_{\mathrm{NLPC}}= & \frac{1}{2} \alpha_{\mathrm{V}} \rho^{2}+\frac{1}{2} \alpha_{\mathrm{TV}} \rho_{3}^{2}+\frac{1}{4} \gamma_{\mathrm{V}} \rho^{4}+\frac{1}{4} \gamma_{\mathrm{TV}} \rho_{3}^{4} \\
& -\eta_{2} \rho_{s}^{2} \rho^{2}-\frac{1}{2} \alpha_{s} \rho_{s}^{2}-\frac{2}{3} \beta_{s} \rho_{s}^{3}-\frac{3}{4} \gamma_{s} \rho_{s}^{4} \\
& -\frac{1}{2} \alpha_{\mathrm{TS}} \rho_{s 3}^{2}+\mathcal{E}_{\mathrm{kin}}^{p}+\mathcal{E}_{\mathrm{kin}}^{n}, \quad \text { and } \\
P_{\mathrm{NLPC}}= & \frac{1}{2} \alpha_{\mathrm{V}} \rho^{2}+\frac{1}{2} \alpha_{\mathrm{TV}} \rho_{3}^{2}+\frac{3}{4} \gamma_{\mathrm{V}} \rho^{4}+\frac{3}{4} \gamma_{\mathrm{TV}} \rho_{3}^{4} \\
& +2 \eta_{1} \rho_{s} \rho^{2}+3 \eta_{2} \rho_{s}^{2} \rho^{2}+2 \eta_{3} \rho_{s} \rho_{3}^{2}+\frac{1}{2} \alpha_{s} \rho_{s}^{2} \\
& +\frac{2}{3} \beta_{s} \rho_{s}^{3}+\frac{3}{4} \gamma_{s} \rho_{s}^{4}+\frac{1}{2} \alpha_{\mathrm{TS}} \rho_{s 3}^{2}+P_{\mathrm{kin}}^{p}+P_{\mathrm{kin}}^{n},
\end{aligned}
$$

respectively. We now define the sixth type of the analyzed parametrizations as follows.

(vi) Type 6 (point-coupling models): Parametrizations of the model described by Eq. (37) in which $\alpha_{\mathrm{TS}}=0$.

For the sake of completeness, we still define a last type of parametrization. (vii) Type 7 ( $\delta$ meson models): Parametrizations of finiterange models presenting the meson $\delta$, i.e., models in which $g_{\delta} \neq 0$ in the respective Lagrangian density and equations of state.

We still calculate the symmetry energy, $\mathcal{S}(\rho)$, for the RMF model shown here from the general definition,

$$
\mathcal{S}(\rho)=\left.\frac{1}{8} \frac{\partial^{2}(\mathcal{E} / \rho)}{\partial y^{2}}\right|_{\rho, y=1 / 2} .
$$

The expressions are

$$
\begin{aligned}
\mathcal{S}_{\mathrm{NL}}(\rho)= & \frac{k_{F}^{2}}{6 E_{F}^{*}}+\frac{g_{\rho}^{2}}{8 m_{\rho}^{* 2}} \rho \\
& -\left(\frac{g_{\delta}}{m_{\delta}}\right)^{2} \frac{M^{* 2} \rho}{2 E_{F}^{* 2}\left[1+\left(g_{\delta} / m_{\delta}\right)^{2} A\left(k_{F}, M^{*}\right)\right]}, \\
\mathcal{S}_{\mathrm{DD}}(\rho)= & \frac{k_{F}^{2}}{6 E_{F}^{*}}+\frac{\Gamma_{\rho}^{2}}{8 m_{\rho}^{2}} \rho \\
& -\left(\frac{\Gamma_{\delta}}{m_{\delta}}\right)^{2} \frac{M^{* 2} \rho}{2 E_{F}^{* 2}\left[1+\left(\Gamma_{\delta} / m_{\delta}\right)^{2} A\left(k_{F}, M^{*}\right)\right]}, \\
\mathcal{S}_{\mathrm{NLPC}}(\rho)= & \frac{k_{F}^{2}}{6 E_{F}^{*}}+\frac{1}{2} \alpha_{\mathrm{TV}} \rho+\eta_{3} \rho_{s} \rho \\
& +\frac{1}{2} \alpha_{\mathrm{TS}} \frac{M^{* 2} \rho}{2 E_{F}^{* 2}\left[1-\alpha_{\mathrm{TS}} A\left(k_{F}, M^{*}\right)\right]},
\end{aligned}
$$

with

$$
\begin{aligned}
E_{F}^{*} & =\left(k_{F}^{2}+M^{* 2}\right)^{1 / 2} \\
A\left(k_{F}, M^{*}\right) & =\frac{2}{\pi^{2}} \int_{0}^{k_{F}} \frac{k^{4} d k}{\left(k^{2}+M^{* 2}\right)^{3 / 2}}, \text { and } \\
m_{\rho}^{* 2} & =m_{\rho}^{2}+g_{\sigma} g_{\rho}^{2} \sigma\left(2 \alpha_{2}+\alpha_{2}^{\prime} g_{\sigma} \sigma\right)+\alpha_{3}^{\prime} g_{\omega}^{2} g_{\rho}^{2} \omega_{0}^{2} .
\end{aligned}
$$

All quantities chosen as constraints that are presented in the next section are directly calculated from $P, \mathcal{E}$, and $\mathcal{S}$, as can be seen from the definitions

$$
\begin{aligned}
K_{0} & =9\left(\frac{\partial P}{\partial \rho}\right)_{\rho=\rho_{0}, y=1 / 2} \quad \text { (incompressibility) } \\
Q_{0} & =\left.27 \rho_{0}^{3} \frac{\partial^{3}(\mathcal{E} / \rho)}{\partial \rho^{3}}\right|_{\rho=\rho_{0}, y=1 / 2} \quad \text { (skewness coefficient) } \\
J & =\mathcal{S}\left(\rho_{0}\right) \quad\left(\text { symmetry energy at } \rho=\rho_{0}\right) \\
L_{0} & =3 \rho_{0}\left(\frac{\partial \mathcal{S}}{\partial \rho}\right)_{\rho=\rho_{0}} \quad(\text { slope of } \mathcal{S}) \\
K_{\text {sym }}^{0} & \left.=9 \rho_{0}^{2}\left(\frac{\partial^{2} \mathcal{S}}{\partial \rho^{2}}\right)_{\rho=\rho_{0}} \quad \text { (curvature of } \mathcal{S}\right) \\
Q_{\text {sym }}^{0} & \left.=27 \rho_{0}^{3}\left(\frac{\partial^{3} \mathcal{S}}{\partial \rho^{3}}\right)_{\rho=\rho_{0}} \quad \text { (skewness of } \mathcal{S}\right)
\end{aligned}
$$




$$
K_{\tau, \mathrm{v}}^{0}=\left(K_{\mathrm{sym}}^{0}-6 L_{0}-\frac{Q_{0}}{K_{0}} L_{0}\right)
$$

(volume part of the isospin incompressibility),

where $\rho_{0}$ is the saturation density; see Ref. [37].

In Appendix A we explicitly calculate some of the quantities defined above as a function of density for RMF models of types 4,5 , and 6 at zero temperature. In the finite-temperature regime, the integrals in $\rho_{p, n}, \rho_{s, n}, \mathcal{E}_{\mathrm{kin}}^{p, n}$, and $P_{\mathrm{kin}}^{p, n}$ should be replaced by those extending from zero to infinity and taking into account the Fermi-Dirac distributions for particles and antiparticles [38].

The saturation properties of all $263 \mathrm{RMF}$ parametrizations analyzed in this work are displayed in Table VII of Appendix B, with the corresponding references.

\section{RESULTS AND DISCUSSIONS}

\section{A. Previous constraints: SET1}

We next present two sets of constraints used to analyze 263 relativistic models. First, we take the same 11 constraints previously used to analyze the Skyrme-type parameterizations. Each individual constraint is explained in detail also in Ref. [5]. Here we summarize them by stressing that they are closely related to properties (i) of SNM: the incompressibility (SM1), skewness coefficient (SM2), density dependence of pressure in the ranges of $2<\frac{\rho}{\rho_{0}}<4.6$ (SM3) and $1.2<\frac{\rho}{\rho_{0}}<2.2$ (SM4); (ii) of pure neutron matter (PNM): the density dependence of energy per particle (PNM1) and pressure (PNM2); (iii) involving both SNM and PNM: the symmetry energy (MIX1), its slope (MIX2), and the volume part of the isospin incompressibility (MIX3), all of them evaluated at the saturation density, the ratio of the symmetry energy at $\rho_{0} / 2$ to its value at $\rho_{0}$ (MIX4), and the ratio of $3 P_{\mathrm{PNM}}\left(\rho_{0}\right)$ to $L_{0} \rho_{0}$ (MIX5). This set of constraints, named here as SET1, is shown in Table I with its respective range of validity.

Regarding specifically pure neutron matter, the related equation of state $(\mathrm{EoS})$ is of a particular interest, because PNM is a realistic first approximation to the baryonic matter that composes neutron stars. Most properties of neutron stars cannot be studied in terrestrial laboratories and theoretical models, based on effective forces, must be used. However, at low densities, experiments with cold Fermi atoms yield information on strongly interacting fluids, very similar to the low-density neutron matter at neutron-star crusts [47]. Different density regimes can be tuned by the magnitude of the neutron Fermi momentum $k_{\mathrm{F}}$ relative to the effective range $r_{\mathrm{o}}$ of the $N N$ interaction in the system [48]. The ground-state energy per particle can be expressed as $E_{\mathrm{PNM}} / E_{\mathrm{PNM}}^{o}=\xi$, where $E_{\mathrm{PNM}}=\mathcal{E}_{\mathrm{PNM}} / \rho$ and $E_{\mathrm{PNM}}^{o}$ is the kinetic part of $E_{\mathrm{PNM}}$. In the dilute degenerate Fermi gas regime $\left(k_{F} r_{\mathrm{o}} \ll 1\right), \xi$ is a constant [49]. This restricts the density below about $10^{-3} \rho_{0}$, the density at which neutrons become unbound in neutron stars. At higher densities, below $\sim 0.1 \rho_{\mathrm{o}}$, where $k_{F} r_{\mathrm{o}} \approx 1, \xi$ has to be replaced by a system-dependent function $\xi\left(k_{\mathrm{F}}, r_{\mathrm{o}}\right)$. Likewise, in Ref. [5], we adopt here the expression $E_{\mathrm{PNM}} / E_{\mathrm{PNM}}^{o}$ by Epelbaum et al. [50], based on next-to-leading order in lattice chiral effective field theory $\left(\mathrm{NLO}_{3}\right)$ and including corrections owing to finite scattering length, nonzero effective range, and higher-order corrections,

$$
\frac{E_{\mathrm{PNM}}}{E_{\mathrm{PNM}}^{o}}=\xi-\frac{\xi_{1}}{k_{F} a_{\mathrm{o}}}+c_{1} k_{F} r_{\mathrm{o}}+c_{2} k_{F}^{2} m_{\pi}^{-2}+c_{3} k_{F}^{3} m_{\pi}^{-3}+\cdots,
$$

where $m_{\pi}$ is the pion mass. The dimensionless universal constant $\xi$ has been determined from trapped cold-atom experiments with ${ }^{6} \mathrm{Li}$ and ${ }^{40} \mathrm{~K}$, which yield a variety of values: $0.32_{-13}^{+10}$ [51], 0.51(4) [52], 0.46-05 [53], and 0.39(2) [54]. Values of $\xi_{1}$ in the literature are in the range 0.8-1.0 ([50] and references therein). Epelbaum et al., using a simple Hamiltonian and only few particles in their system, took $\xi=0.31$ and $\xi_{1}=$ 0.81 and fitted two sets of constants, namely $\left(c_{1}=0.27, c_{2}=\right.$ $\left.-0.44, c_{3}=0.0\right)$ and $\left(c_{1}=0.17, c_{2}=0.0, c_{3}=-0.26\right)$, and obtained a very similar quality fit to their $\mathrm{NLO}_{3}$. We used the constraint on energy per particle of PNM in the range of densities $0.01-0.1 \rho_{\mathrm{o}}$ showed in Figs. 1(c) and 3(c) with $\xi_{1}=0.81$ and the two sets $\left(c_{1}, c_{2}, c_{3}\right)$. The band is obtained by taking $0.2<\xi<0.6$, which allows for the spread in experimental values. This is the constraint named as PNM1.

TABLE I. List of the macroscopic constraints of SET1 and the range of their experimental and empirical (exp/emp) values, density region

\begin{tabular}{|c|c|c|c|c|c|}
\hline Constraint & Quantity & Density region & $\begin{array}{l}\text { Range of constraint } \\
\text { (exp/emp) }(\mathrm{MeV})\end{array}$ & $\begin{array}{l}\text { Range of constraint } \\
\text { from CRMF }(\mathrm{MeV})\end{array}$ & Reference \\
\hline SM1 & $K_{0}$ & $\rho_{0}\left(\mathrm{fm}^{-3}\right)$ & $200-260$ & 271.0 & [39] \\
\hline SM3 & $P(\rho)$ & $2<\frac{\rho}{\rho_{0}}<4.6$ & Band region & see Fig. 1 & [41] \\
\hline SM4 & $P(\rho)$ & $1.2<\frac{\rho}{\rho_{0}}<2.2$ & Band region & see Fig. 1 & {$[42]$} \\
\hline PNM1 & $\mathcal{E}_{\mathrm{PNM}} / \rho$ & $0.017<\frac{\rho}{\rho_{0}}<0.108$ & Band region & see Fig. 1 & {$[5]$} \\
\hline MIX1 & $J$ & $\rho_{0}\left(\mathrm{fm}^{-3}\right)$ & $30-35$ & $33.8-34.0$ & {$[43]$} \\
\hline MIX2 & $L_{0}$ & $\rho_{0}\left(\mathrm{fm}^{-3}\right)$ & $40-76$ & $70.9-73.9$ & [44] \\
\hline MIX3 & $K_{\tau, \mathrm{v}}^{0}$ & $\rho_{0}\left(\mathrm{fm}^{-3}\right)$ & -760 to -372 & -388.5 to -388.4 & [10] \\
\hline MIX4 & $\frac{\mathcal{S}\left(\rho_{0} / 2\right)}{J}$ & $\rho_{0}\left(\mathrm{fm}^{-3}\right)$ & $0.57-0.86$ & 0.58 & [45] \\
\hline MIX5 & $\frac{3 P_{\mathrm{PNM}}^{\gamma}}{L_{0} \rho_{0}}$ & $\rho_{0}\left(\mathrm{fm}^{-3}\right)$ & $0.90-1.10$ & $1.05-1.06$ & [46] \\
\hline
\end{tabular}
in which they are valid, and the corresponding range obtained using the approved RMF models (CRMF). 

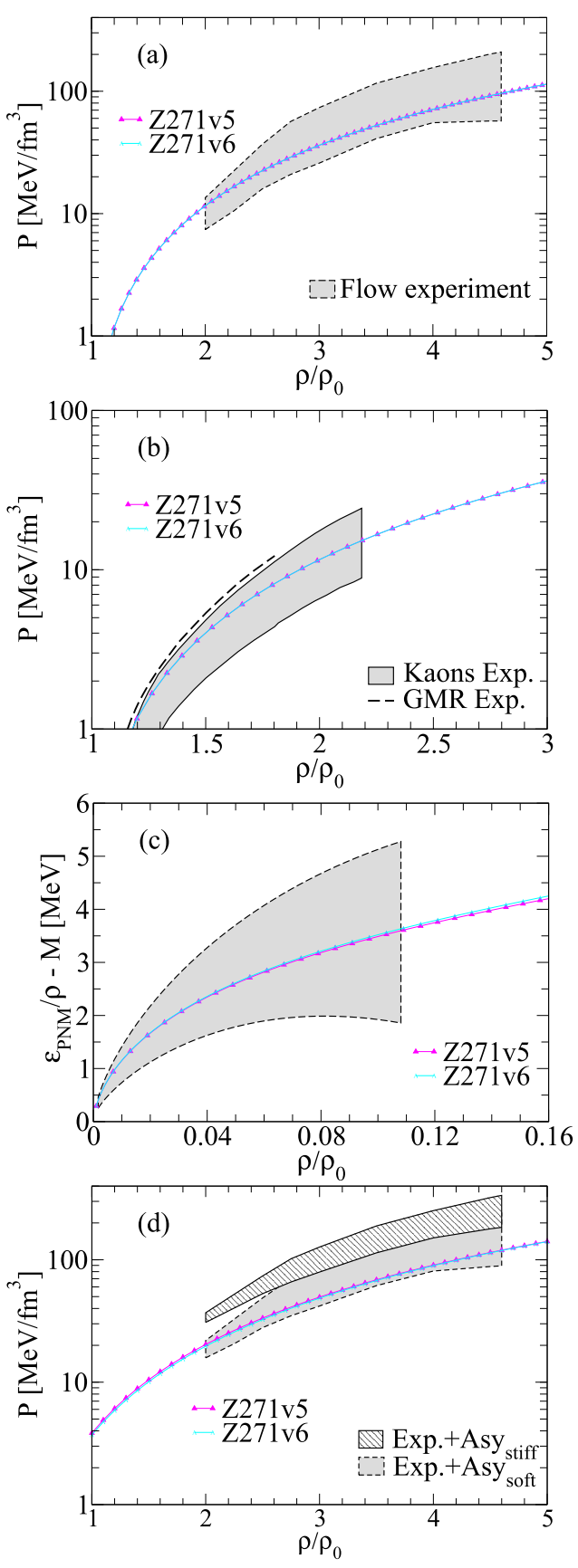

FIG. 1. (Color online) Density dependence of the approved models Z271v5 and Z271v6 in the (a) SM3, (b) SM4, (c) PNM1, and (d) PNM2 constraints related to SET1. The shaded bands were extracted from Ref. [41], where flow experimental data is compared with results obtained for (a) symmetric matter and (d) pure neutron matter, (b) Ref. [42], where pressure in symmetric matter is compared with data extracted from kaon production, and (c) Ref. [5], as explained in the text.

As in Ref. [5], a model is considered approved in a numerical constraint if its deviation, given as

$$
\operatorname{dev}=\frac{Q_{\mathrm{mod}}-Q_{\mathrm{const}}}{\Delta}
$$

TABLE II. List of parametrizations that fail in only one constraint of SET1

\begin{tabular}{lcc}
\hline \hline Model & Model value $(\mathrm{MeV})$ & Deviation \\
\hline Constraint not satisfied: SM1 $\left(200 \leqslant K_{0} \leqslant 260 \mathrm{MeV}\right)$ & \\
Z271v5 & 271.00 & 1.37 \\
Z271v6 & 271.00 & 1.37 \\
Constraint not satisfied: MIX3 $\left(-760 \leqslant K_{\tau, \mathrm{v}}^{0} \leqslant-372 \mathrm{MeV}\right)$ \\
BSR15 & -252.54 & 1.62 \\
BSR16 & -258.75 & 1.58 \\
FSUGold & -276.07 & 1.49 \\
FSUGZ06 & -259.47 & 1.58 \\
FSUGold4 & -205.59 & 1.86 \\
FSU-III & -341.03 & 1.16 \\
FSU-IV & -210.68 & 1.83 \\
TW99 & -332.32 & 1.20 \\
DD-F & -285.54 & 1.45 \\
DD-ME $\delta$ & -258.28 & 1.59 \\
\hline \hline
\end{tabular}

obeys the relation $|\operatorname{dev}| \leqslant 1$, with $Q_{\text {mod }}$ being the value of the quantity calculated in the model, $Q_{\text {const }}$ the central value of the related constraint, and $\Delta$ the error related to $Q_{\text {const }}$. Specifically for the MIX1, MIX3, and MIX4 constraints, we define their central values as $Q_{\text {const }}=\left(x_{2}+x_{1}\right) / 2$ and the error as $\Delta=x_{2}-Q_{\text {const }}=Q_{\text {const }}-x_{1}$, because they are given in the form of $x_{1} \leqslant X \leqslant x_{2}$. However, a graphic constraint is satisfied if the model is inside the corresponding band in $95 \%$ or more of the density region.

We also present in the fifth column of Table I the range of the quantities used in each constraint obtained from the approved models which are designated by consistent relativistic mean field (CRMF). This range is defined from the smaller and larger values of the respective quantity chosen among the selected models. For instance, the range of the slope of the symmetry energy, $70.9 \leqslant L_{0} \leqslant 73.9$, is constructed by noting that $L_{0}^{\mathrm{Z} 271 \mathrm{v} 6}=70.9 \mathrm{MeV}$ and $L_{0}^{\mathrm{Z} 271 \mathrm{v} 5}=73.9 \mathrm{MeV}$. For the graphic constraints, we analyze the density dependence of the Z271v5 and Z271v6 parameterizations in Fig. 1.

By applying the constraints of SET1 to the RMF parametrizations, we obtain the result that none of the models satisfies all constraints simultaneously. However, there are 12 parametrizations that do not satisfy only one individual constraint; i.e., they are consistent with the 10 remaining ones. We specify such parametrizations in Table II, including the constraint they do not satisfy and the respective deviation obtained from Eq. (59).

In these 12 parametrizations, 2 specific ones, models of type 4 (cross-terms models), namely, Z271v5 and Z271v6, fall outside the limits of the SM1 constraint (the one not satisfied only for these two models) by less than $5 \%$; i.e., their incompressibilities, $K_{0}^{271 v 5}=K_{0}^{271 v 6}=271 \mathrm{MeV}$, exceed the highest value of the SM1 constraint, $K_{0 . S M 1}^{\mathrm{max}}=260 \mathrm{MeV}$, by $11 \mathrm{MeV}$ and the ratio of this excess to $K_{0, \mathrm{SM} 1}=230 \mathrm{MeV}$ is less than $5 \%$. For such cases, we apply the same criterion of Ref. [5] and define these models as included in the CRMF parameterizations, i.e., the ones satisfying the constraints of SET1. 
TABLE III. Number of approved models (among 263) in each constraint of SET1.

\begin{tabular}{lccccccccrrr}
\hline \hline Constraints & SM1 & SM2 & SM3 & SM4 & PNM1 & PNM2 & MIX1 & MIX2 & MIX3 & MIX4 & MIX5 \\
\hline No. of models & 146 & 174 & 104 & 153 & 193 & 101 & 162 & 59 & 124 & 65 & 258 \\
\hline \hline
\end{tabular}

For the sake of completeness, we provide in Table III the number of the RMF approved models for each constraint of SET1.

It is worth noting that the MIX5 constraint is the "weakest" one, while the constraint defined by the slope of the symmetry energy, MIX2, is the "strongest" among all of them, because only 58 of 263 parametrizations present $L_{0}$ in the range of $L_{0}=58 \pm 18 \mathrm{MeV}$.

\section{B. Updated constraints: SET2a and SET2b}

It is well known that some of the validity ranges are different if obtained for Skyrme-type or relativistic models. Several studies involving both RMF models and nonrelativistic models have shown that although both sets of models verify the same correlations, the parameter distribution of each set is not completely overlapping; see, for instance, Refs. [15,27,37]. This is a consequence of the different structure of these models, namely, the existence of a scalar density completely absent in the nonrelativistic models which gives contributions corresponding to many-body effects [55]. In fact, the constraints imposed within each of the sets are not measured directly, but they result from the analysis of raw data, which involves model assumptions. As an example, we refer that the incompressibility derived from GMR using Skyrme interactions is around $230 \mathrm{MeV}$, but many of the RMF models predict higher values (see, e.g., Ref. [10]). In a similar way, a value $J=32.5 \pm$ $0.5 \mathrm{MeV}$ is found by fitting a large set of experimental data in the finite-range droplet-model (FRDM) [56]. The extrapolation of the various fits for the nonrelativistic models (Skyrme and Gogny) yield typical values of $J$ in the region of 27 to $38 \mathrm{MeV}$. Also, for such a quantity, the various RMF parametrizations yield higher values; see Fig. 18 of Ref. [43] for a clear comparison. Based on this phenomenology, we have opted for other sets of constraints that are somewhat more adequate to analyze the RMF models. In these new sets, named hereafter as SET2a and SET2b, we also intend to take into account new theoretical, experimental, and empirical information concerning the quantities related to the constraints used in this work.

First of all, we discuss the SM1 constraint, related to the incompressibility of infinite nuclear matter. In the recent study of Refs. [11,12], the authors investigated the density dependence of the incompressibility in various relativistic and nonrelativistic models, finding a crossing point around a density of $\rho_{c}=0.7 \rho_{0}$. They pointed out the existence of this crossing also in other bulk properties such as the symmetry energy [23] and the energy density of pure neutron matter [57]. In fact, phenomenological models are generally fitted to finite nuclei which provide fitting constraints at a density slightly below saturation, making $\rho_{c}$ more suitable to characterize nuclear finite systems. In this perspective, the authors have shown that the quantity $M_{c}=\left.3 \rho_{c} \frac{\partial K(\rho)}{\partial \rho}\right|_{\rho=\rho_{c}}$ is more strongly correlated with $E_{\mathrm{GMR}}$ than $K_{0}=K\left(\rho_{0}\right)$ and claimed to use this relation to first constrain $M_{c}$ and after to infer the value of $K_{0}$ ( $E_{\mathrm{GMR}}$ is the centroid energy of the isoscalar giant monopole resonance). This is an alternative to the often-used method of constraining $K_{0}$ directly from its correlation with $E_{\mathrm{GMR}}$. Following this new approach, and by using experimental data of $E_{\mathrm{GMR}}$ for ${ }^{208} \mathrm{~Pb},{ }^{112-124} \mathrm{Sn}$ isotopes, ${ }^{90} \mathrm{Zr}$, and ${ }^{144} \mathrm{Sm}$, the linear correlation of $E_{\mathrm{GMR}}$ and $M_{c}$ shown in Refs. [11,12] was used to constrain $M_{c}$ to the range $M_{c}=1100 \pm 70 \mathrm{MeV}$. Thereby, a new range of $K_{0}$ was proposed by noting that $K_{0}$ and $M_{c}$ are also linearly correlated [11,12]. In our new set of updated constraints, we use this range of values for $K_{0}$ as our new SM1 constraint, in this case given by $K_{0}=230 \pm$ $40 \mathrm{MeV}$. Notice that this new constraint is slightly less restrictive than the old one given by $K_{0}=230 \pm 30 \mathrm{MeV}$.

Although interesting, we note that the new constraint is based on theory and a specific selection of Skyrme forces (with the exception of FSUGold, DDME2, and D1S which seem to cluster just at the low $M_{c}$ values). The selection of experimental $E_{\mathrm{GMR}}$ is also limited; there are many more values $E_{\mathrm{GMR}}$ in the literature which would have to be used to verify the conclusion of Fig. 3 (Fig. 2) in Ref. [11] (Ref. [12]). For example, there are three different sets of the experimental $E_{\mathrm{GMR}}$ in ${ }^{208} \mathrm{~Pb}$ which differ outside errors: $13.91 \pm 0.11 \mathrm{MeV}$, $13.90 \pm 0.30 \mathrm{MeV}, 14.24 \mathrm{MeV}, 14.17 \pm 0.28 \mathrm{MeV}, 14.18 \pm$ $0.11 \mathrm{MeV}$ (see discussion in Ref. [39]), $13.96 \pm 0.20 \mathrm{MeV}$ [58], $13.4 \pm 0.2 \mathrm{MeV}$ [59], and 13.5 $\pm 0.2 \mathrm{MeV}$ [60]. If the lowest value, 13.2 MeV, allowed by the error was used, the constraint on $M_{c}$ would be different. Also, the error on $E_{\mathrm{GMR}}$ ${ }^{120} \mathrm{Sn}$ is $200 \mathrm{keV}$, not $100 \mathrm{keV}(15.4 \pm 0.2 \mathrm{MeV})[28,61]$.

Another constraint directly established from a correlation with the incompressibility is the SM2 one. In Ref. [40], the authors used the leptodermous expansion for the incompressibility of a finite nucleus of mass number $A$ and radius $R$,

$$
\begin{aligned}
K(A, y)= & K_{0}+K_{\text {surf }} A^{-1 / 3}+K_{\text {curv }} A^{-2 / 3}+K_{\tau, \mathrm{v}}^{0}(1-2 y)^{2} \\
& +K_{\text {coul }} \frac{Z^{2}}{A^{4 / 3}}+\cdots,
\end{aligned}
$$

and its relation with $E_{\mathrm{GMR}}$ through

$$
K(A, y)=\left(M / \hbar^{2}\right)\left\langle R^{2}\right\rangle E_{\mathrm{GMR}}^{2},
$$

to find the range of $K^{\prime}=700 \pm 500 \mathrm{MeV}$ [the volume part of the isospin incompressibility, $K_{\tau, \mathrm{v}}^{0}$, is written in terms of $K^{\prime}=-Q_{0}$ in Eq. (57)]. This constraint was obtained from the range predicted for the incompressibility, $K_{0}=$ $215 \pm 15 \mathrm{MeV}$, extracted from the comparison of experimental values of $E_{\mathrm{GMR}}$ and those calculated from theoretical models. However, the analysis of Ref. [40] was entirely based on parameterizations of the nonrelativistic Skyrme model. For this reason, and because of the difference between $K_{0}$ found in Ref. [40] and the one defined in our new SM1 constraint, 
we have decided here to eliminate the SM2 constraint from SET2 and, consequently, from the updated analysis of the RMF parametrizations.

The SM3 constraint is related to the limits of the density dependence of the pressure of INM. In the previous work [5], we have taken these limits as defined by Danielewicz and coauthors in Ref. [41]. In that work, the authors established such limits from analysis of transverse and elliptical flows of the ejected particles in the ${ }^{197} \mathrm{Au}+{ }^{197} \mathrm{Au}$ collisions.

In a recent study [62], Steiner et al. extracted the radius $r$ of a $1.4 M_{\odot}$ neutron star in the range $10.4 \leqslant r \leqslant 12.9 \mathrm{~km}$, generating a new constraint that equations of state must satisfy for the mass-radius relation of neutron stars. Their analysis was based on observational data of (i) bursting neutron stars showing photospheric radius expansion and of (ii) transiently accreting neutron stars in quiescence. As a consequence, the authors also established a new range of validity for the density dependence of the pressure of infinity nuclear matter, consistent with the previous SM3 constraint proposed in Ref. [41] in the lower pressure region. In the high-pressure region, however, the new constraint is broader than the former. To take into account this new phenomenology, we use here the SM3 constraint in two different levels, namely, the SM3a, in which we consider the band in the density dependence of pressure increased by $20 \%$ in its upper limit, and the SM3b, in which we use the band exactly as in the SM3 constraint of SET1. It is worth noting that the SM3a constraint is a less restrictive version of the SM3 one used in SET1.

We note that very recently the radius of a $1.4 M_{\odot}$ neutron star [62] has been updated by Lattimer and Steiner [63] to be 11.15$12.66 \mathrm{~km}$ (with $95 \%$ confidence) if a nucleon-only equation of state is considered and 10.45-12.45 km for an equation of state with exotic components. Their analysis was performed on exactly the same five objects as the analysis reported by Guillot et al. [64], who found the radius of a wide range of neutron-star masses to be $9.1_{-1.5}^{+1.3} \mathrm{~km}$ (with $90 \%$ confidence). Further observation and analysis would be desirable to refine constraints related to mass radius of cold neutron stars.

In Ref. [41], Danielewicz et al., also proposed a constraint on the density dependence of the pressure in pure neutron matter. They made an extrapolation of data concerning transversal and elliptical flow of the previous case by including asymmetry terms with strong and weak density dependence in the pressure. This constraint, named PNM2, was used in Ref. [5] and also in SET1. However, in SET2 we decide not to consider it, by understanding that no new experimental information on heavy-ion collisions is present in such an extrapolation. Therefore, the PNM2 constraint is absent from SET2.

Concerning the constraints on the symmetry energy at the saturation density $(J)$, we decide to consider in SET2 the fact that effective hadronic models present values for $J$ in a broader range than that used in SET1, namely, $30 \leqslant J \leqslant 35 \mathrm{MeV}$. We consider here a small modification in the lower limit of this range, giving rise to our MIX1a constraint in which $25 \leqslant J \leqslant 35 \mathrm{MeV}$. By the same token, we modify the range of the slope of the symmetry energy at $\rho_{0}\left(L_{0}\right)$ to the new one given by $25 \leqslant L_{0} \leqslant 115 \mathrm{MeV}$. This constraint is named MIX2a. However, we call the reader's attention to the fact that

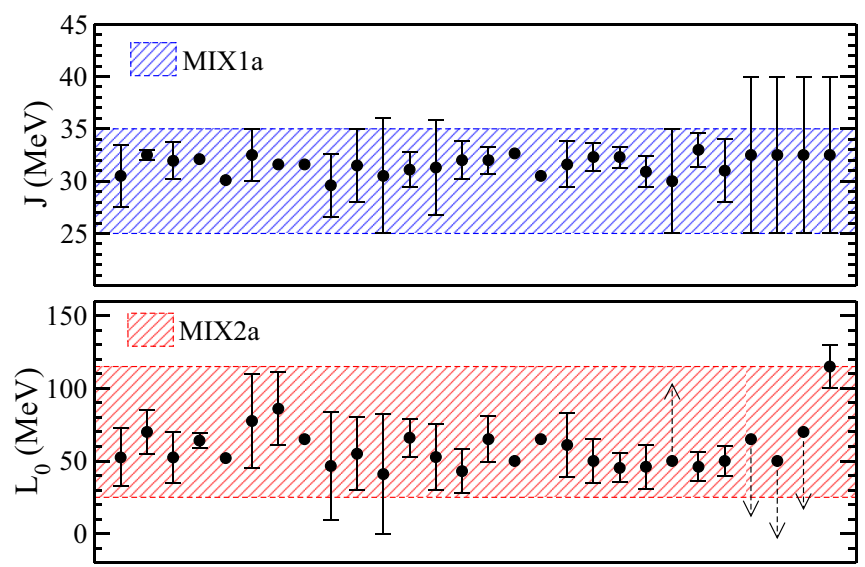

FIG. 2. (Color online) Comparison between the limits used in MIX1a and MIX2a constraints and those from 28 different experimental and observational data collected in Ref. [65].

the new limits established for MIX1a and MIX2a are totally compatible with experimental values available in the literature. To make this clear, we present in Fig. 2, a set of $28 \mathrm{~J}$ and $L$ values extracted from Ref. [65], in which the authors collected from the literature data obtained from analyses of different terrestrial nuclear experiments and astrophysical observations. They include analyses of isospin diffusion, neutron skins, pygmy dipole resonances, $\alpha$ and $\beta$ decays, transverse flow, the mass-radius relation of neutron stars, and torsional crust oscillations of neutron stars. As one can see in Fig. 2, the new constraints encompass all experimental and observational data.

The reader is reminded that this comparison, based on searching for an overlap of the outcome of many experimental and observational methods, inherently implies that all of them have the same weight, which may not be in consensus in the literature. However, we wish to make clear that this comparison has been used in this work only as a guideline, to take into account the more recent data regarding the possible ranges of validity for $J$ and $L$.

We also compare the broader range of $J$ with our previous one of Ref. [5], named in SET2 as MIX1b, and, for the sake of completeness, we also compare the new range of $L_{0}$ with a combination of the ranges recently given in Refs. $[18,66]$. We name this more restrictive range for $L_{0}$, namely, $30 \leqslant L_{0} \leqslant$ $80 \mathrm{MeV}$, as MIX2b.

To finish the list of updated constraints, we make several remarks concerning the limits of the quantity $K_{\tau, \mathrm{v}}^{0}$. First of all, we point out here that such a quantity is extremely relevant in our study, because it represents the volume part of the isospin incompressibility. It is important to analyze the values that RMF models predict for this observable, in the sense that such an investigation can offer a clue for the improvement of the isospin part of effective RMF interactions. According to the literature, there are at least three methods for finding a constraint on the value of $K_{\tau}$, defined as $K_{\tau}=K_{\tau, \mathrm{v}}^{0}+K_{\tau, \mathrm{s}}$, where the last term is related to the surface part of the isospin incompressibility. In Ref. [26], the authors used an isospin- and momentum-dependent transport model to obtain the degree of isospin diffusion in the ${ }^{124} \mathrm{Sn}+{ }^{112} \mathrm{Sn},{ }^{124} \mathrm{Sn}+{ }^{124} \mathrm{Sn}$, and 
TABLE IV. The same as in Table I, now taking into account the following updated constraints: SM1, SM3a, SM3b, MIX1a, MIX1b, MIX2a, MIX2b, and MIX3. The SM4, PNM1, and MIX4 constraints are the same as in SET1. The SM2, PNM2, and MIX5 constraints were removed. These new constraints are used to generate two new sets, namely, SET2a and SET2b (see the text for their definitions).

\begin{tabular}{|c|c|c|c|c|c|}
\hline Constraint & Quantity & Density region & $\begin{array}{l}\text { Range of constraint } \\
\text { (exp/emp) }(\mathrm{MeV})\end{array}$ & $\begin{array}{l}\text { Range of constraint } \\
\text { from CRMF (MeV) }\end{array}$ & Ref. \\
\hline SM1 & $K_{0}$ & $\rho_{0}\left(\mathrm{fm}^{-3}\right)$ & $190-270$ & $225.2-232.4$ & {$[11,12]$} \\
\hline SM3b & $P(\rho)$ & $2<\frac{\rho}{\rho_{0}}<5$ & Band region & see Fig. 3 & [41] \\
\hline SM4 & $P(\rho)$ & $1.2<\frac{\rho}{\rho_{0}}<2.2$ & Band region & see Fig. 3 & [42] \\
\hline PNM1 & $\mathcal{E}_{\mathrm{PNM}} / \rho$ & $0.017<\frac{\rho}{\rho_{0}}<0.108$ & Band region & see Fig. 3 & {$[5]$} \\
\hline MIX1b & $J$ & $\rho_{0}\left(\mathrm{fm}^{-3}\right)$ & $30-35$ & $33.2-34.0$ & [43] \\
\hline MIX2a & $L_{0}$ & $\rho_{0}\left(\mathrm{fm}^{-3}\right)$ & $25-115$ & $77.9-84.8$ & \\
\hline MIX2b & $L_{0}$ & $\rho_{0}\left(\mathrm{fm}^{-3}\right)$ & $30-80$ & $77.9-78.8$ & {$[18,66]$} \\
\hline MIX3 & $K_{\tau, \mathrm{v}}^{0}$ & $\rho_{0}\left(\mathrm{fm}^{-3}\right)$ & -700 to -400 & $-421.6(\mathrm{a}) /-414.3(\mathrm{~b})$ to $-382.5 \mathrm{MeV}$ & \\
\hline MIX4 & $\frac{\mathcal{S}\left(\rho_{0} / 2\right)}{J}$ & $\rho_{0}\left(\mathrm{fm}^{-3}\right)$ & $0.57-0.86$ & $0.57(\mathrm{a}) / 0.59(\mathrm{~b})-0.59$ & [45] \\
\hline
\end{tabular}

${ }^{112} \mathrm{Sn}+{ }^{112} \mathrm{Sn}$ collisions, at energies of $50 \mathrm{MeV} /$ nucleon and impact parameter of $6 \mathrm{fm}$. The correlation between this degree of diffusion and the isospin incompressibility led to the constraint of $-500 \pm 50 \mathrm{MeV}$ for $K_{\tau}$. However, Centelles et al. found in Ref. [27] that $c_{\mathrm{sym}}\left(\rho \simeq 0.1 \mathrm{fm}^{-3}\right)=a_{\text {sym }}(A)$, where $c_{\text {sym }}(\rho) \simeq J-L_{0} \epsilon+\frac{1}{2} K_{\text {sym }}^{0} \epsilon^{2}$ (with $\left.\epsilon=\frac{\rho_{0}-\rho}{3 \rho_{0}}\right)$ and the symmetry energy coefficient of finite nuclei is $a_{\text {sym }}(A)=$ $J /\left[1+(9 J / 4 \mathcal{Q}) A^{1 / 3}\right](\mathcal{Q}$ is the surface stiffness). From this relation, and using that $a_{\mathrm{sym}}(A)$ is a linear function of the neutron skin thickness, the authors estimated a range of $K_{\tau}=-500_{-100}^{+125} \mathrm{MeV}$ by analyzing the neutron skin thickness of 26 antiprotonic atoms. Finally, in Ref. [28], the authors used an expression for $K(A, y)$ similar to Eq. (60) to obtain the value of $K_{\tau}=-550 \pm 100 \mathrm{MeV}$ from data of $E_{\mathrm{GMR}}$.

Regarding the theoretical calculations for $K_{\tau}$, it is also important to mention here that mean-field models predict only its volume term, i.e., $K_{\tau, \mathrm{v}}^{0}$. However, as it is rather tricky to separate the volume from the surface term (although it has been done several times; see, e.g., Ref. [10] and references therein), and as the volume term seems to be dominant, we have opted to constrain the calculated volume term with $K_{\tau}$ values obtained from experiment; i.e., we have assumed $K_{\tau, \mathrm{v}}^{0} \approx K_{\tau}$ for the RMF models. Finally, to take into account all aforementioned procedures for the estimation of $K_{\tau}$, we define here our new constraint MIX3 as $K_{\tau}=-550 \pm 150 \mathrm{MeV}$.

Finally, we stress here that the MIX5 constraint was also removed from our new analysis because it is a very "weak" constraint, in the sense that practically all models are approved in its range; see Table III. Thus, we do not consider it as a good model selector.

The list of the updated constraints together with those not modified from SET1 is given in Table IV.

From these updated constraints we define two distinct sets: one of them composed by the constraints in which their ranges are broader, SET2a, and other one in which they are more stringent, namely, SET2b. Specifically, SET2a (SET2b) is defined by the SM1, SM3a (SM3b), SM4, PNM1, MIX1a (MIX1b), MIX2a (MIX2b), MIX3, and MIX4 constraints.

The application of the constraints of SET2a to the 263 RMF parametrizations collected in our work leads to only two models satisfying all constraints simultaneously. They are the type 4 models BSR12 and BKA24. In addition, 24 parametrizations satisfy all constraints of SET2a except one; i.e., they are approved in seven of the eight constraints. In this group, the BSR11 and BKA22 models, also of type 4, fell outside the range of the unsatisfied constraint (in this case, MIX3) by less than 5\%. Therefore, as we have done in Ref. [5], and in the previous analysis of SET1, we consider these four models as belonging to the CRMF models of SET2a.

When considering the SET2b, the more stringent one, we found no significant changes in the results. In such an analysis, only the BSR12 model, among 263, satisfies all SET2b simultaneously, and 22 parametrizations satisfy seven of the eight constraints. Two such models fell outside the range of the unsatisfied constraint by less than 5\%, namely, BSR 11 and BKA22, the same as in the SET2a analysis. The MIX3 constraint is again the one not satisfied for these two models. In summary, we have, in total, three models consistent with the SET2b analysis.

It is worth noting that the difference between the two analyses regarding SET2a and SET2b is the absence of the BKA24 model in the CRMF approved models of SET2b, in comparison to those approved in SET2a. The reason for this absence is the slope of the symmetry energy of the BKA24 model, $L_{0}=84.8 \mathrm{MeV}$, clearly outside the MIX2b range, given by $L_{0}=55 \pm 25 \mathrm{MeV}$, by more than $5 \%$.

As in the previous section, we present in the fifth column of Table IV the range of the constraints of SET2, defined by the CRMF models. In the cases of MIX3 and MIX4 constraints, the boundary models are different for both sets, namely, SET2a and SET2b. Therefore, we included the letter (a) or (b) in the values to identify the corresponding set. The density dependence of the approved models in both analyses is shown in Fig. 3.

Also regarding our analysis of SET2a and SET2b, in Table $\mathrm{V}$ we present the number of approved models in each constraint.

We also provide in Table VIII of Appendix C the information whether each model of the 263 ones is approved $(+)$ or not (-) in the constraints of SET2. In Table IX of Appendix D, 

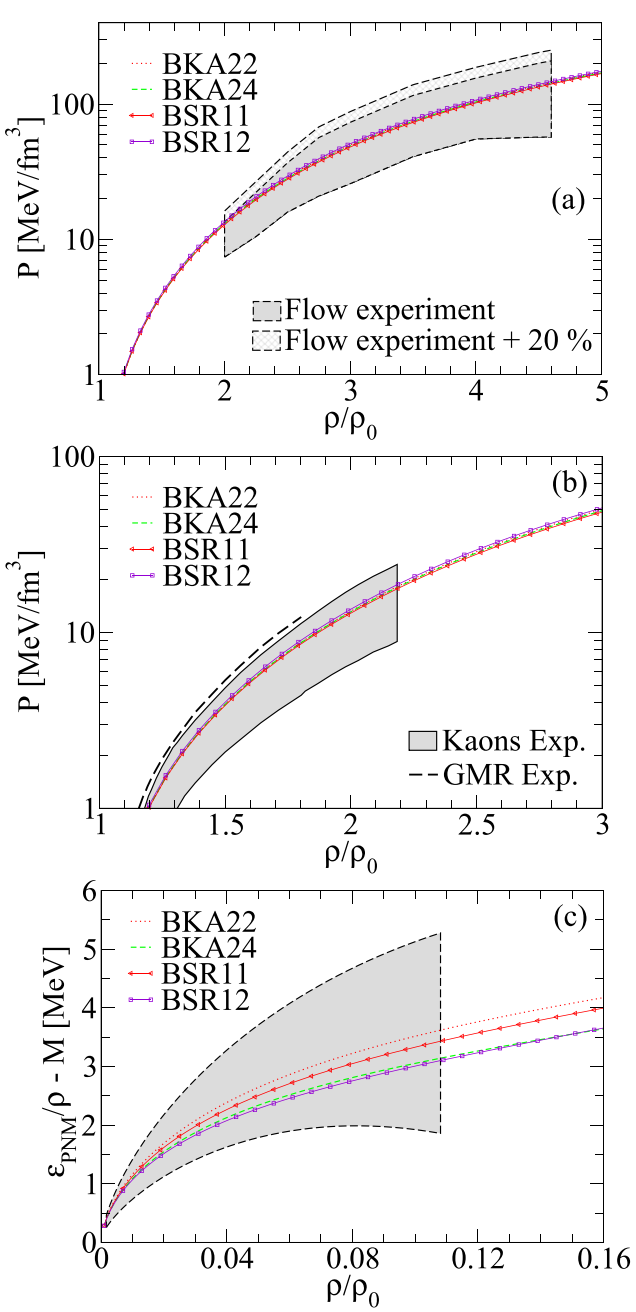

FIG. 3. (Color online) Density dependence of approved models in the (a) SM3, (b) SM4, and (c) PNM1 constraints related to SET2a and SET2b. Shaded bands extracted from (a) Ref. [41], where flow experimental data are compared with results obtained for symmetric matter, (b) Ref. [42], where pressure in symmetric matter is compared with data extracted from kaon production, and (c) Ref. [5], as explained in the text.

we give the deviation as calculated in Eq. (59) for the numerical constraints, as well as those obtained for the graphic constraints, all of them related to SET2.

To finish the analysis of SET2, we point out that our work concerns only nuclear matter. Owing to the translational invariance, rotational symmetry, and rotational invariance around the third axis in isospin space of the latter, we can constrain only some features of the Lagrangian and the approved models cannot guarantee successful predictions for finite nuclei. From our analysis, one can see from Table VIII that the models FSUGold, FSUZG03, DD-ME $\delta$, and IU-FSU are consistent with all constraints except MIX3, a constraint applicable in the region of saturation density (it applies only to SET2a for the IU-FSU model). These parameter sets provide quite good global fits to binding energies, charge radii, isotopic shifts, and neutron skin thicknesses.

For the sake of completeness, we display in Table VI several nuclear matter properties, at the saturation density, of the approved models in all sets analyzed here, namely, SET1, SET2a, and SET2b.

\section{Excluding MIX3 constraint}

The results presented in the previous section pointed to a small number of RMF models consistent to all constraints simultaneously, namely, four in the SET2a analysis and three for the SET $2 b$ analysis. However, these numbers change significantly if we simply discard the MIX3 constraint from SET2a and SET2b. By excluding this constraint, the new results for the SET2a analysis become the following: 25 models are consistent with all seven constraints. They are: BKA20, BKA22, BKA24, BSR8, BSR9, BSR10, BSR11, BSR12, BSR15, BSR16, BSR17, BSR18, BSR19, DD-ME $\delta$, DD-F, DDH $\delta$, FSU-III, FSU-IV, FSUGold, FSUGold4, FSUGZ03, FSUGZ06, G2*, IU-FSU, and TW99. Moreover, 48 models satisfy all but one of the constraints. In this group, 10 models fell outside the range of the constraint by less than $5 \%$. They are BSR20, FA3, Z271s2, Z271s3, Z271s4, Z271s5, Z271s6, Z271v4, Z271v5, and Z271v6. By also including such models in the CRMF parameterizations, one has 35 models consistent with the SET2a analysis.

For the SET2b, we have 22 models consistent with all constraints. These are the same models approved in SET2a, except for the BKA24, IU-FSU, and DDH $\delta$ models. Moreover, 14 models satisfy only seven constraints, and by applying the 5\% criterion, 8 more models are approved. They are the same as in the corresponding case of SET2a, except for the BSR20 and FA3 models. Therefore, one has in the SET2b analysis a total of 30 models in the group of approved models.

\section{Additional new constraints}

After the work presented in this paper has been completed, new constraints from reanalysis of data on GMR energies became available [10], suggesting that the SM1 constraint would span to somewhat higher values, $250-315 \mathrm{MeV}$ (SM1new) and MIX3 would be between -620 and $-370 \mathrm{MeV}$ (MIX3-new). We reanalysed the data introducing the two additional constraints with the following results.

TABLE V. Number of approved models (among 263) in each constraint of SET2.

\begin{tabular}{lccccccccccc}
\hline \hline \multicolumn{10}{c}{ SET2 } \\
\hline Constraints & SM1 & SM3a & SM3b & SM4 & PNM1 & MIX1a & MIX1b & MIX2a & MIX2b & MIX3 & MIX4 \\
No. of models & 153 & 129 & 104 & 153 & 193 & 174 & 162 & 216 & 72 & 96 & 65 \\
\hline \hline
\end{tabular}


TABLE VI. Nuclear matter properties at the saturation density $\rho_{0}$ of the RMF models consistent with the macroscopic constraints.

\begin{tabular}{|c|c|c|c|c|c|c|c|c|c|c|}
\hline Model & Approved in SET & $\begin{array}{c}\rho_{0} \\
\left(\mathrm{fm}^{-3}\right)\end{array}$ & $\begin{array}{c}E_{0} \\
(\mathrm{MeV})\end{array}$ & $\begin{array}{c}K_{0} \\
(\mathrm{MeV})\end{array}$ & $m^{*}$ & $\begin{array}{c}K^{\prime} \\
(\mathrm{MeV})\end{array}$ & $\begin{array}{c}J \\
(\mathrm{MeV})\end{array}$ & $\begin{array}{c}L_{0} \\
(\mathrm{MeV})\end{array}$ & $\begin{array}{c}K_{\tau, \mathrm{v}}^{0} \\
(\mathrm{MeV})\end{array}$ & $\frac{\mathcal{S}\left(\rho_{0} / 2\right)}{J}$ \\
\hline BKA22 & $2 a$ and $2 b$ & 0.147 & -15.91 & 225.24 & 0.61 & 283.29 & 33.17 & 78.79 & -382.46 & 0.59 \\
\hline BSR11 & $2 a$ and $2 b$ & 0.147 & -16.08 & 226.75 & 0.61 & 312.37 & 33.69 & 78.78 & -388.86 & 0.59 \\
\hline BSR12 & $2 a$ and $2 b$ & 0.147 & -16.10 & 232.35 & 0.61 & 290.31 & 34.00 & 77.90 & -414.30 & 0.59 \\
\hline Z271v5 & 1 & 0.148 & -16.24 & 271.00 & 0.80 & 733.59 & 34.04 & 73.90 & -388.52 & 0.58 \\
\hline
\end{tabular}

\section{Analysis considering MIX3-new in SET2}

(i) SET2a (SM1-new, SM3a, SM4, PNM1, MIX1a, MIX2a, MIX3-new, and MIX4): Four models approved, namely, Z271v4, Z271v5, Z271v6, and FA3; 24 models not satisfying only one constraint. Even with $5 \%$ tolerance, no further model approved.

(ii) SET2b (SM1-new, SM3b, SM4, PNM1, MIX1b, MIX2b, MIX3-new, and MIX4): Three models approved, namely, Z271v4, Z271v5, and Z271v6; 12 models not satisfying only one constraint. Even with $5 \%$ tolerance, no further model approved.

\section{Analysis discarding MIX3-new in SET2}

(i) SET2a (SM1-new, SM3a, SM4, PNM1, MIX1a, MIX2a, and MIX4): Nine models approved, namely, Z271v4, Z271v5, Z271v6, Z271s2, Z271s3, Z271s4, Z271s5, Z271s6, and FA3; 45 models not satisfying only one constraint. Even with 5\% tolerance, no further model approved.

(ii) SET2b (SM1-new, SM3b, SM4, PNM1, MIX1b, MIX2b, and MIX4): Eight models approved, namely, Z271v4, Z271v5, Z271v6, Z271s2, Z271s3, Z271s4, Z271s5, and Z271s6; 26 models not satisfying only one constraint. Even with 5\% tolerance, no further model approved.

\section{SUMMARY AND CONCLUSIONS}

In this work we submitted 263 parametrizations of the widely used RMF hadronic models to three different sets of constraints. One of them (SET1) is composed exactly of the same constraints used in the extensive study of Ref. [5] in which 240 parametrizations of the nonrelativistic Skyrme model were examined. The second one, named SET2, was divided in two other sets, in which updated constraints were taken into account and some of them removed from our analysis. The first set, SET2a, contains a broader version of the constraints and the second, SET2b, a more stringent one. In summary, all sets present constraints regarding information of SNM, pure neutron matter (PNM), and those in which these two frameworks are considered simultaneously (MIX), i.e., constraints derived from the symmetry energy at the saturation density.

We have organized the RMF models in seven different groups regarding their Lagrangian density structure, namely, linear (type 1), $\sigma^{3}+\sigma^{4}$ (type 2), $\sigma^{3}+\sigma^{4}+\omega_{0}^{4}$ (type 3), $\sigma^{3}+\sigma^{4}+\omega_{0}^{4}+$ cross terms (type 4 ), density-dependent (type 5 ), point-coupling (type 6), and $\delta$ meson (type 7) models. Their saturation properties are displayed in Table VII of Appendix B.

The application of the SET1 constraints to the models leads to the impressive result of only the Z271v5 and Z271v6 parametrizations being classified as consistent RMF (CRMF) models. This result is still more stringent than that found in Ref. [5] in which among 240 Skyrme parametrizations, only 16 were approved in the analysis regarding SET1. If we consider our analysis based on the constraints of SET2a, a more updated version in comparison with SET1, the results do not change significantly. In this case, BKA22, BKA24, BSR11, and BSR12 are the CRMF models, and, except for the BKA24 model, this is the same result when we use the SET2b, a more stringent one in comparison with the updated SET2a. The saturation properties of these models are summarized in Table VI. Also, the density dependence of the properties used to construct the graphic constraints are shown in Figs. 1 and 3.

As an interesting feature concerning our analysis, we point out that in all sets of constraints, the approved models are of type 4, i.e., models presenting cross terms between the mesonic fields $\sigma, \omega_{\mu}$, and $\overrightarrow{\rho_{\mu}}$. In particular, these interactions include a density dependence of the symmetry energy that goes beyond the almost linear behavior of models of the types 1, 2, and 3 . Moreover, models BKA22 and BKA24 yield a neutron-skin thickness in the ${ }^{208} \mathrm{~Pb}$ nucleus, respectively, of 0.22 and $0.24 \mathrm{fm}$ and were found to be consistent with most of the constraints included in sets $2 \mathrm{a}$ and $2 \mathrm{~b}$ [67]. This means that models of type 4 , those that include the cross-term interactions, favor the reproduction of the expected behavior of the properties defined in each individual constraint. To satisfy the constraints used in this work, new parameterizations of the RMF models must take into account the density dependence of the symmetry energy through the inclusion of cross terms between the isoscalar mesons and the vector-isovector meson or other means.

We also highlight that such results change dramatically if we simply neglect from our analysis in SET2a and SET2b the constraint regarding the volume part of the isospin incompressibility, $K_{\tau, \mathrm{v}}^{0} \approx K_{\tau}=-550 \pm 150 \mathrm{MeV}$. By applying the seven remaining constraints of SET2a, we found the following 35 approved models: BKA20, BKA22, BKA24, BSR8, BSR9, BSR10, BSR11, BSR12, BSR15, BSR16, BSR17, BSR18, BSR19, DD-ME $\delta$, DD-F, DDH $\delta$, FSU-III, FSU-IV, FSUGold, FSUGold4, FSUGZ03, FSUGZ06, G2*, IU-FSU, TW99, BSR20, FA3, Z271s2, Z271s3, Z271s4, Z271s5, Z271s6, Z271v4, Z271v5, and Z271v6. Notice that, besides the 
cross-term models, other types of models are consistent with the SET2 a constraints, namely, two density-dependent (DD-F, TW99), one point-coupling (FA3), and two $\delta$ meson models (DD-ME $\delta, \mathrm{DDH} \delta$ ). The common feature of these models is that they do not include the simple symmetry energy density dependence present in all type 1, 2, and 3 models. The use of isospin-dependent properties in the fit procedure is essential to get a reasonable parametrization. For SET2b, the analysis leads to the same group of approved models excluding the BKA24, IU-FSU, DDH $\delta$, BSR20, and FA3 models, totaling 30 consistent parametrizations. Notice that in this more restrictive set, one of the $\delta$ meson (DDH $\delta$ ) models and the point-coupling model are excluded from the CRMF models. However, the density-dependent models DD-F and TW99 remain approved.

In the present work we have used the known physics of nuclear matter at densities around the saturation value to constrain relativistic models. However, these models have been extensively used to describe compact star constituents and macroscopic properties, such as radii and masses, and for this purpose have been extended to much higher densities. In this respect, the inclusion of other degrees of freedom, such as the lightest eight baryons, is energetically favored, but the onset of hyperons softens the equations of state and consequently reduces the maximum stellar masses [68]. Having in mind the description of two recently detected neutron stars with masses of the order of $2 M_{\odot}[69,70]$, appropriate equations of state with the inclusion of hyperons were shown to strongly depend on the choice of the models and also on the hyperon-meson coupling constants [71-73]. After the comprehensive analyses performed in the present work, we suggest that the models that passed all tests be used in further studies involving the inclusion of hyperons in astrophysical applications.

We remark that there is still another class of relativistic models known as quark-meson-coupling models [74], in which baryons are described as a system of nonoverlapping MIT bags interacting through intermediate mesons. In these models, quark degrees of freedom are explicitly taken into account and the couplings are determined at the quark level. These models will also be examined according to the constraints proposed in the present work in a future investigation.

Finally, we reinforce that our work represents a unique effort to qualify the current RMFs and their usefulness for modeling of nuclear matter, which has never been done before. At present it is impossible to critically compare published results, obtained with a different selection of RMF models because nuclear matter properties are model dependent. Consistent use of a narrowed selection of approved RMF model should allow to improve the situation. Furthermore, the failure of many RMF models to satisfy the most up-to-date constraints should stimulate search for missing physics, which, when included, should lead to improvement of the models and their predictive power.

As a final remark concerning the analysis performed in the present work (RMF models), and in the previous one (nonrelativistic Skyrme models [5]), we highlight that it would be desirable to establish a unique protocol to study the predictive power of mean-field models (both relativistic and nonrelativistic) to deal not only with nuclear matter but also with finite nuclei. Actually, Stone and Reinhard outlined a possible protocol for the Skyrme interaction in Sec. 5 of their review [43] by indicating the finite nuclei constraints that should be used to select Skyrme parametrizations, such as charge rms radius, spin-orbit splittings, neutron radii isotopic shifts, excitation properties, and others. Despite the fact that this procedure was developed for nonrelativistic models, it should also be applicable to RMF ones. Some of the entries should be updated, but the basic philosophy remains the same. One of the positive outcomes of such a work would be to learn more about surface properties of finite nuclei. It is well known that the crucial difference between finite nuclei and nuclear matter is the presence (or absence) of the nuclear surface, which plays a very important role, but is not yet well understood. Comparison of the properties of nuclear matter around saturation density and those of finite nuclei could yield valuable information in this direction. A preliminary study involving these ideas was performed in Ref. [75] for the Skyrme parametrizations selected in Ref. [5]. A more detailed study, also taking into account the selected RMF models presented here, will be addressed in a future work.

\section{ACKNOWLEDGMENTS}

This work was partially supported by CNPq (Brazil), CAPES (Brazil), and FAPESP (Brazil) under Project No. 2009/00069-5 and FAPESC (Brazil) under Project No. 2716/2012,TR 2012000344; by COMPETE/FEDER and FCT (Portugal) under Grant No. PTDC/FIS/113292/2009; and by NEW COMPSTAR, a COST initiative. M.D. acknowledges the hospitality at ITA/CTA and D.P.M. acknowledges the hospitality at the Universidad de Alicante, where parts of this work were carried out. S.T. is grateful for the support by the Helmholtz Association (HGF) through the Nuclear Astrophysics Virtual Institute (Grant No. VH-VI-417). J.R.S. wishes to thank D.M.'s group at Universidade Federal de Santa Catarina for its hospitality during her stay, made possible by CNPq Processo (Grant No. 401593/2009-6), when this project was initiated. O.L. acknowledges support from FAPESP.

\section{APPENDIX A: ANALYTICAL EXPRESSIONS FOR INFINITE NUCLEAR MATTER QUANTITIES}

In this Appendix, we show the analytical expressions of some quantities at $y=1 / 2$ used to analyze the constraints of Sec. III related to RMF models of types 4 (nonlinear finite range), 5 (density dependent), and 6 (NLPC). The quantities are obtained at zero temperature and generalized to any density.

\section{Finite-range models}

The incompressibility is obtained as

$$
\begin{aligned}
K_{\mathrm{NL}}= & 9\left(\frac{\partial P_{\mathrm{NL}}}{\partial \rho}\right)_{y=1 / 2} \\
= & 9\left[g_{\omega} \rho \frac{\partial \omega_{0}}{\partial \rho}+\frac{k_{F}^{2}}{3\left(k_{F}^{2}+M^{* 2}\right)^{1 / 2}}\right. \\
& \left.+\frac{\rho M^{*}}{\left(k_{F}^{2}+M^{* 2}\right)^{1 / 2}} \frac{\partial M^{*}}{\partial \rho}\right],
\end{aligned}
$$


where

$$
\frac{\partial M^{*}}{\partial \rho}=-g_{\sigma} \frac{\partial \sigma}{\partial \rho}
$$

The skewness coefficient is

$$
\begin{aligned}
Q_{\mathrm{NL}} & =\left.27 \rho^{3} \frac{\partial^{3}\left(\mathcal{E}_{\mathrm{NL}} / \rho\right)}{\partial \rho^{3}}\right|_{y=1 / 2} \\
& =27 \rho^{3}\left[\frac{1}{\rho} \frac{\partial^{3} \mathcal{E}_{\mathrm{NL}}}{\partial \rho^{3}}-\frac{3}{\rho^{2}} \frac{\partial^{2} \mathcal{E}_{\mathrm{NL}}}{\partial \rho^{2}}+\frac{6}{\rho^{3}} \frac{\partial \mathcal{E}_{\mathrm{NL}}}{\partial \rho}-\frac{6 \mathcal{E}_{\mathrm{NL}}}{\rho^{4}}\right]_{y=1 / 2},
\end{aligned}
$$

with

$$
\begin{gathered}
\frac{\partial \mathcal{E}_{\mathrm{NL}}}{\partial \rho}=\left(k_{F}^{2}+M^{* 2}\right)^{1 / 2}+g_{\omega} \omega_{0}, \\
\frac{\partial^{2} \mathcal{E}_{\mathrm{NL}}}{\partial \rho^{2}}=g_{\omega} \frac{\partial \omega_{0}}{\partial \rho}+\frac{1}{2 E_{F}^{*}}\left(\frac{\pi^{2}}{k_{F}}+2 M^{*} \frac{\partial M^{*}}{\partial \rho}\right), \text { and } \\
\frac{\partial^{3} \mathcal{E}_{\mathrm{NL}}}{\partial \rho^{3}}=g_{\omega} \frac{\partial^{2} \omega_{0}}{\partial \rho^{2}}-\frac{1}{4 E_{F}^{* 3}}\left(\frac{\pi^{2}}{k_{F}}+2 M^{*} \frac{\partial M^{*}}{\partial \rho}\right)^{2} \\
+\frac{1}{2 E_{F}^{*}}\left[-\frac{\pi^{4}}{2 k_{F}^{4}}+2\left(\frac{\partial M^{*}}{\partial \rho}\right)^{2}+2 M^{*} \frac{\partial^{2} M^{*}}{\partial \rho^{2}}\right] .
\end{gathered}
$$

The quantities $\mathcal{E}_{\mathrm{NL}}, \frac{\partial \mathcal{E}_{\mathrm{NL}}}{\partial \rho}, \frac{\partial^{2} \mathcal{E}_{\mathrm{NL}}}{\partial \rho^{2}}$, and $\frac{\partial^{3} \mathcal{E}_{\mathrm{NL}}}{\partial \rho^{3}}$ are evaluated at $y=1 / 2$.

The slope and curvature of symmetry energy are given, respectively, by

$$
L_{\mathrm{NL}}=3 \rho\left(\frac{\partial \mathcal{S}_{\mathrm{NL}}}{\partial \rho}\right)=\frac{k_{F}^{2}}{3 E_{F}^{*}}-\frac{k_{F}^{4}}{6 E_{F}^{* 3}}\left(1+\frac{2 M^{*} k_{F}}{\pi^{2}} \frac{\partial M^{*}}{\partial \rho}\right)+\frac{3 g_{\rho}^{2}}{8 m_{\rho}^{* 2}} \rho-\frac{3 g_{\rho}^{2}}{8 m_{\rho}^{* 4}} \frac{\partial m_{\rho}^{* 2}}{\partial \rho} \rho^{2}
$$

and

$$
\begin{aligned}
K_{\mathrm{sym}}^{\mathrm{NL}}= & 9 \rho^{2}\left(\frac{\partial^{2} \mathcal{S}_{\mathrm{NL}}}{\partial \rho^{2}}\right)=9 \rho^{2}\left\{-\frac{\pi^{2}}{12 E_{F}^{* 3} k_{F}}\left(\frac{\pi^{2}}{k_{F}}+2 M^{*} \frac{\partial M^{*}}{\partial \rho}\right)-\frac{\pi^{4}}{12 E_{F}^{*} k_{F}^{4}}-\frac{g_{\rho}^{2}}{4 m_{\rho}^{* 4}} \frac{\partial m_{\rho}^{* 2}}{\partial \rho}\right. \\
& -\left[\frac{\pi^{4}}{24 E_{F}^{* 3} k_{F}^{2}}-\frac{k_{F} \pi^{2}}{8 E_{F}^{* 5}}\left(\frac{\pi^{2}}{k_{F}}+2 M^{*} \frac{\partial M^{*}}{\partial \rho}\right)\right]\left(1+\frac{2 M^{*} k_{F}}{\pi^{2}} \frac{\partial M^{*}}{\partial \rho}\right)+\frac{g_{\rho}^{2}}{4 m_{\rho}^{* 6}}\left(\frac{\partial m_{\rho}^{* 2}}{\partial \rho}\right)^{2} \rho \\
& \left.-\frac{k_{F} \pi^{2}}{12 E_{F}^{* 3}}\left[\frac{M^{*}}{k_{F}^{2}} \frac{\partial M^{*}}{\partial \rho}+\frac{2 k_{F}}{\pi^{2}}\left(\frac{\partial M^{*}}{\partial \rho}\right)^{2}+\frac{2 k_{F} M^{*}}{\pi^{2}} \frac{\partial^{2} M^{*}}{\partial \rho^{2}}\right]-\frac{g_{\rho}^{2}}{8 m_{\rho}^{* 4}} \frac{\partial^{2} m_{\rho}^{* 2}}{\partial \rho^{2}} \rho\right\} .
\end{aligned}
$$

For SNM, where $y=1 / 2$ and $\bar{\rho}_{0(3)}=0$, the density derivatives of $\sigma$ and $\omega_{0}$ are given by

$$
\frac{\partial \sigma}{\partial \rho}=\frac{a_{1} b_{2}+a_{2} b_{3}}{a_{1} b_{1}-a_{3} b_{3}} \quad \text { and } \quad \frac{\partial \omega_{0}}{\partial \rho}=\frac{a_{2} b_{1}+a_{3} b_{2}}{a_{1} b_{1}-a_{3} b_{3}}
$$

where

$$
\begin{aligned}
a_{1}= & m_{\omega}^{2}+3 c g_{\omega}^{4} \omega_{0}^{2}+g_{\sigma} g_{\omega}^{2} \sigma\left(2 \alpha_{1}+\alpha_{1}^{\prime} g_{\sigma} \sigma\right), \\
a_{2}= & g_{\omega}, \\
a_{3}= & -2 g_{\sigma} g_{\omega}^{2} \omega_{0}\left(\alpha_{1}+\alpha_{1}^{\prime} g_{\sigma} \sigma\right), \\
b_{1}= & m_{\sigma}^{2}+2 A \sigma+3 B \sigma^{2}-g_{\sigma}^{2} g_{\omega}^{2} \omega_{0}^{2} \alpha_{1}^{\prime} \\
& +3 g_{\sigma}^{2}\left(\frac{\rho_{s}}{M^{*}}-\frac{\rho}{E_{F}^{*}}\right),
\end{aligned}
$$

$$
\begin{aligned}
& b_{2}=\frac{g_{\sigma} M^{*}}{E_{F}^{*}}, \text { and } \\
& b_{3}=-a_{3} .
\end{aligned}
$$

For the sake of completeness, we also present the proton and neutron chemical potentials,

$$
\begin{aligned}
\mu_{p}^{\mathrm{NL}}= & \frac{\partial \mathcal{E}_{\mathrm{NL}}}{\partial \rho_{p}}=\left(k_{F}^{2}+M^{* 2}\right)^{1 / 2}+g_{\omega} \omega_{0} \\
& +\frac{g_{\rho}}{2} \bar{\rho}_{0(3)} \quad \text { and } \\
\mu_{n}^{\mathrm{NL}}= & \frac{\partial \mathcal{E}_{\mathrm{NL}}}{\partial \rho_{n}}=\left(k_{F}^{2}+M^{* 2}\right)^{1 / 2}+g_{\omega} \omega_{0}-\frac{g_{\rho}}{2} \bar{\rho}_{0(3)}
\end{aligned}
$$

both at any density and proton fraction $y$. 


\section{Density-dependent models}

The incompressibility is

$$
\begin{aligned}
K_{\mathrm{DD}} & =9\left(\frac{\partial P_{\mathrm{DD}}}{\partial \rho}\right)_{y=1 / 2} \\
& =9\left[\rho \frac{\partial \Sigma_{R}}{\partial \rho}+\omega_{0} \rho \frac{\partial \Gamma_{\omega}}{\partial \rho}+\Gamma_{\omega} \rho \frac{\partial \omega_{0}}{\partial \rho}+\frac{k_{F}^{2}}{3\left(k_{F}^{2}+M^{* 2}\right)^{1 / 2}}+\frac{\rho M^{*}}{\left(k_{F}^{2}+M^{* 2}\right)^{1 / 2}} \frac{\partial M^{*}}{\partial \rho}\right] \\
& =9\left[\rho \frac{\partial \Sigma_{R}}{\partial \rho}+\frac{2 \Gamma_{\omega} \rho^{2}}{m_{\omega}^{2}} \frac{\partial \Gamma_{\omega}}{\partial \rho}+\frac{\Gamma_{\omega}^{2} \rho}{m_{\omega}^{2}}+\frac{k_{F}^{2}}{3\left(k_{F}^{2}+M^{* 2}\right)^{1 / 2}}+\frac{\rho M^{*}}{\left(k_{F}^{2}+M^{* 2}\right)^{1 / 2}} \frac{\partial M^{*}}{\partial \rho}\right],
\end{aligned}
$$

with

$$
\begin{aligned}
\frac{\partial M^{*}}{\partial \rho} & =-\left(\Gamma_{\sigma} \frac{\partial \sigma}{\partial \rho}+\sigma \frac{\partial \Gamma_{\sigma}}{\partial \rho}\right) \quad \text { and } \\
\frac{\partial \sigma}{\partial \rho} & =\frac{\left[\rho_{s}-3\left(\frac{\rho_{s}}{M^{*}}-\frac{\rho}{E_{F}^{*}}\right) \Gamma_{\sigma} \sigma\right] \frac{\partial \Gamma_{\sigma}}{\partial \rho}+\frac{\Gamma_{\sigma} M^{*}}{E_{F}^{*}}}{m_{\sigma}^{2}+3\left(\frac{\rho_{s}}{M^{*}}-\frac{\rho}{E_{F}^{*}}\right) \Gamma_{\sigma}^{2}}
\end{aligned}
$$

observing that $\omega_{0}=\frac{\Gamma_{\omega} \rho}{m_{\omega}^{2}}$ and $\sigma=\frac{\Gamma_{\sigma} \rho_{s}}{m_{\sigma}^{2}}$.

The skewness coefficient is

$$
Q_{\mathrm{DD}}=\left.27 \rho^{3} \frac{\partial^{3}\left(\mathcal{E}_{\mathrm{DD}} / \rho\right)}{\partial \rho^{3}}\right|_{y=1 / 2}=27 \rho^{3}\left[\frac{1}{\rho} \frac{\partial^{3} \mathcal{E}_{\mathrm{DD}}}{\partial \rho^{3}}-\frac{3}{\rho^{2}} \frac{\partial^{2} \mathcal{E}_{\mathrm{DD}}}{\partial \rho^{2}}+\frac{6}{\rho^{3}} \frac{\partial \mathcal{E}_{\mathrm{DD}}}{\partial \rho}-\frac{6 \mathcal{E}_{\mathrm{DD}}}{\rho^{4}}\right]_{y=1 / 2},
$$

with

$$
\begin{aligned}
\frac{\partial \mathcal{E}_{\mathrm{DD}}}{\partial \rho}= & \left(k_{F}^{2}+M^{* 2}\right)^{1 / 2}+\frac{\Gamma_{\omega}^{2}}{m_{\omega}^{2}} \rho+\bar{\Sigma}_{R} \\
\frac{\partial^{2} \mathcal{E}_{\mathrm{DD}}}{\partial \rho^{2}}= & \frac{1}{2 E_{F}^{*}}\left(\frac{\pi^{2}}{k_{F}}+2 M^{*} \frac{\partial M^{*}}{\partial \rho}\right)+\frac{\Gamma_{\omega}^{2}}{m_{\omega}^{2}}+\frac{2 \Gamma_{\omega} \rho}{m_{\omega}^{2}} \frac{\partial \Gamma_{\omega}}{\partial \rho}+\frac{\partial \bar{\Sigma}_{R}}{\partial \rho}, \text { and } \\
\frac{\partial^{3} \mathcal{E}_{\mathrm{DD}}}{\partial \rho^{3}}= & -\frac{1}{4 E_{F}^{* 3}}\left(\frac{\pi^{2}}{k_{F}}+2 M^{*} \frac{\partial M^{*}}{\partial \rho}\right)^{2}+\frac{1}{2 E_{F}^{*}}\left[-\frac{\pi^{4}}{2 k_{F}^{4}}+2\left(\frac{\partial M^{*}}{\partial \rho}\right)^{2}+2 M^{*} \frac{\partial^{2} M^{*}}{\partial \rho^{2}}\right] \\
& +\frac{2 \Gamma_{\omega} \rho}{m_{\omega}^{2}} \frac{\partial^{2} \Gamma_{\omega}}{\partial \rho^{2}}+\frac{2 \rho}{m_{\omega}^{2}}\left(\frac{\partial \Gamma_{\omega}}{\partial \rho}\right)^{2}+\frac{4 \Gamma_{\omega}}{m_{\omega}^{2}} \frac{\partial \Gamma_{\omega}}{\partial \rho}+\frac{\partial^{2} \bar{\Sigma}_{R}}{\partial \rho^{2}}
\end{aligned}
$$

where

$$
\begin{aligned}
& \bar{\Sigma}_{R}=\Sigma_{R}(y=1 / 2)=\frac{\Gamma_{\omega} \rho^{2}}{m_{\omega}^{2}} \frac{\partial \Gamma_{\omega}}{\partial \rho}-\frac{\Gamma_{\sigma} \rho_{s}^{2}}{m_{\sigma}^{2}} \frac{\partial \Gamma_{\sigma}}{\partial \rho}, \\
& \frac{\partial^{2} M^{*}}{\partial \rho^{2}}=-\left(\Gamma_{\sigma} \frac{\partial^{2} \sigma}{\partial \rho^{2}}+\sigma \frac{\partial^{2} \Gamma_{\sigma}}{\partial \rho^{2}}\right)-2 \frac{\partial \Gamma_{\sigma}}{\partial \rho} \frac{\partial \sigma}{\partial \rho}, \\
& \frac{\partial^{2} \sigma}{\partial \rho^{2}}=\left[\left(\rho_{s}-\mathcal{J} \Gamma_{\sigma} \sigma\right) \frac{\partial^{2} \Gamma_{\sigma}}{\partial \rho^{2}}+\left(\frac{\partial \rho_{s}}{\partial \rho}-\Gamma_{\sigma} \sigma \frac{\partial \mathcal{J}}{\partial \rho}-\mathcal{J} \sigma \frac{\partial \Gamma_{\sigma}}{\partial \rho}-\mathcal{J} \Gamma_{\sigma} \frac{\partial \sigma}{\partial \rho}\right) \frac{\partial \Gamma_{\sigma}}{\partial \rho}+\frac{M^{*}}{E_{F}^{*}} \frac{\partial \Gamma_{\sigma}}{\partial \rho}\right. \\
& \left.+\frac{\Gamma_{\sigma}}{E_{F}^{*}} \frac{\partial M^{*}}{\partial \rho}-\frac{\Gamma_{\sigma} M^{*}}{E_{F}^{* 2}} \frac{\partial E_{F}^{*}}{\partial \rho}\right]\left(m_{\sigma}^{2}+\mathcal{J} \Gamma_{\sigma}^{2}\right)^{-1}-\left[\left(\rho_{s}-\mathcal{J} \Gamma_{\sigma} \sigma\right) \frac{\partial \Gamma_{\sigma}}{\partial \rho}+\frac{\Gamma_{\sigma} M^{*}}{E_{F}^{*}}\right]\left[\frac{\partial \mathcal{J}}{\partial \rho} \Gamma_{\sigma}^{2}+2 \mathcal{J} \Gamma_{\sigma} \frac{\partial \Gamma_{\sigma}}{\partial \rho}\right]\left(m_{\sigma}^{2}+\mathcal{J} \Gamma_{\sigma}^{2}\right)^{-2}, \\
& \mathcal{J}=3\left(\frac{\rho_{s}}{M^{*}}-\frac{\rho}{E_{F}^{*}}\right), \\
& \frac{\partial E_{F}^{*}}{\partial \rho}=\frac{\pi^{2}}{2 E_{F}^{*} k_{F}}\left(1+\frac{2 M^{*} k_{F}}{\pi^{2}} \frac{\partial M^{*}}{\partial \rho}\right)
\end{aligned}
$$




$$
\begin{aligned}
\frac{\partial^{2} \bar{\Sigma}_{R}}{\partial \rho^{2}}= & \frac{\Gamma_{\omega} \rho^{2}}{m_{\omega}^{2}} \frac{\partial^{3} \Gamma_{\omega}}{\partial \rho^{3}}+\frac{4 \Gamma_{\omega} \rho}{m_{\omega}^{2}} \frac{\partial^{2} \Gamma_{\omega}}{\partial \rho^{2}}+\frac{3 \rho^{2}}{m_{\omega}^{2}} \frac{\partial \Gamma_{\omega}}{\partial \rho} \frac{\partial^{2} \Gamma_{\omega}}{\partial \rho^{2}}+\frac{4 \rho}{m_{\omega}^{2}}\left(\frac{\partial \Gamma_{\omega}}{\partial \rho}\right)^{2}+\frac{2 \Gamma_{\omega}}{m_{\omega}^{2}} \frac{\partial \Gamma_{\omega}}{\partial \rho} \\
& -\frac{\Gamma_{\sigma} \rho_{s}^{2}}{m_{\sigma}^{2}} \frac{\partial^{3} \Gamma_{\sigma}}{\partial \rho^{3}}-\frac{4 \Gamma_{\sigma} \rho_{s}}{m_{\sigma}^{2}} \frac{\partial \rho_{s}}{\partial \rho} \frac{\partial^{2} \Gamma_{\sigma}}{\partial \rho^{2}}-\frac{3 \rho_{s}^{2}}{m_{\sigma}^{2}} \frac{\partial \Gamma_{\sigma}}{\partial \rho} \frac{\partial^{2} \Gamma_{\sigma}}{\partial \rho^{2}}-\frac{4 \rho_{s}}{m_{\sigma}^{2}} \frac{\partial \rho_{s}}{\partial \rho}\left(\frac{\partial \Gamma_{\sigma}}{\partial \rho}\right)^{2} \\
& -\frac{2 \Gamma_{\sigma}}{m_{\sigma}^{2}}\left(\frac{\partial \rho_{s}}{\partial \rho}\right)^{2} \frac{\partial \Gamma_{\sigma}}{\partial \rho}-\frac{2 \Gamma_{\sigma} \rho_{s}}{m_{\sigma}^{2}} \frac{\partial^{2} \rho_{s}}{\partial \rho^{2}} \frac{\partial \Gamma_{\sigma}}{\partial \rho}, \\
\frac{\partial \rho_{s}}{\partial \rho}= & \frac{M^{*}}{E_{F}^{*}}-\mathcal{J}\left(\sigma \frac{\partial \Gamma_{\sigma}}{\partial \rho}+\Gamma_{\sigma} \frac{\partial \sigma}{\partial \rho}\right),
\end{aligned}
$$

and

$$
\frac{\partial^{2} \rho_{s}}{\partial \rho^{2}}=\frac{1}{E_{F}^{*}} \frac{\partial M^{*}}{\partial \rho}-\frac{M^{*}}{E_{F}^{* 2}} \frac{\partial E_{F}^{*}}{\partial \rho}-\mathcal{J}\left(\sigma \frac{\partial^{2} \Gamma_{\sigma}}{\partial \rho^{2}}+2 \frac{\partial \Gamma_{\sigma}}{\partial \rho} \frac{\partial \sigma}{\partial \rho}+\Gamma_{\sigma} \frac{\partial^{2} \sigma}{\partial \rho^{2}}\right)-\frac{\partial \mathcal{J}}{\partial \rho}\left(\sigma \frac{\partial \Gamma_{\sigma}}{\partial \rho}+\Gamma_{\sigma} \frac{\partial \sigma}{\partial \rho}\right) .
$$

The slope and curvature of $\mathcal{S}_{\mathrm{DD}}$ are

$$
L_{\mathrm{DD}}=3 \rho\left(\frac{\partial \mathcal{S}_{\mathrm{DD}}}{\partial \rho}\right)=\frac{k_{F}^{2}}{3 E_{F}^{*}}-\frac{k_{F}^{4}}{6 E_{F}^{* 3}}\left(1+\frac{2 M^{*} k_{F}}{\pi^{2}} \frac{\partial M^{*}}{\partial \rho}\right)+\frac{3 \Gamma_{\rho}^{2}}{8 m_{\rho}^{2}} \rho+\frac{3 \Gamma_{\rho}}{4 m_{\rho}^{2}} \frac{\partial \Gamma_{\rho}}{\partial \rho} \rho^{2}
$$

and

$$
\begin{aligned}
K_{\mathrm{sym}}^{\mathrm{DD}}= & 9 \rho^{2}\left(\frac{\partial^{2} \mathcal{S}_{\mathrm{DD}}}{\partial \rho^{2}}\right)=9 \rho^{2}\left\{-\frac{\pi^{2}}{12 E_{F}^{* 3} k_{F}}\left(\frac{\pi^{2}}{k_{F}}+2 M^{*} \frac{\partial M^{*}}{\partial \rho}\right)-\frac{\pi^{4}}{12 E_{F}^{*} k_{F}^{4}}+\frac{\Gamma_{\rho}}{2 m_{\rho}^{2}} \frac{\partial \Gamma_{\rho}}{\partial \rho}\right. \\
& -\left[\frac{\pi^{4}}{24 E_{F}^{* 3} k_{F}^{2}}-\frac{k_{F} \pi^{2}}{8 E_{F}^{* 5}}\left(\frac{\pi^{2}}{k_{F}}+2 M^{*} \frac{\partial M^{*}}{\partial \rho}\right)\right]\left(1+\frac{2 M^{*} k_{F}}{\pi^{2}} \frac{\partial M^{*}}{\partial \rho}\right)+\frac{\rho}{4 m_{\rho}^{2}}\left(\frac{\partial \Gamma_{\rho}}{\partial \rho}\right)^{2} \\
& \left.-\frac{k_{F} \pi^{2}}{12 E_{F}^{* 3}}\left[\frac{M^{*}}{k_{F}^{2}} \frac{\partial M^{*}}{\partial \rho}+\frac{2 k_{F}}{\pi^{2}}\left(\frac{\partial M^{*}}{\partial \rho}\right)^{2}+\frac{2 k_{F} M^{*}}{\pi^{2}} \frac{\partial^{2} M^{*}}{\partial \rho^{2}}\right]+\frac{\Gamma_{\rho} \rho}{4 m_{\rho}^{2}} \frac{\partial^{2} \Gamma_{\rho}}{\partial \rho^{2}}\right\} .
\end{aligned}
$$

The chemical potentials for any proton fraction are

$$
\begin{aligned}
& \mu_{p}^{\mathrm{DD}}=\frac{\partial \mathcal{E}_{\mathrm{DD}}}{\partial \rho_{p}}=\left(k_{F}^{2}+M^{* 2}\right)^{1 / 2}+\Gamma_{\omega} \omega_{0}+\frac{\Gamma_{\rho}}{2} \bar{\rho}_{0(3)}+\Sigma_{R} \quad \text { and } \\
& \mu_{n}^{\mathrm{DD}}=\frac{\partial \mathcal{E}_{\mathrm{DD}}}{\partial \rho_{n}}=\left(k_{F}^{2}+M^{* 2}\right)^{1 / 2}+\Gamma_{\omega} \omega_{0}-\frac{\Gamma_{\rho}}{2} \bar{\rho}_{0(3)}+\Sigma_{R} .
\end{aligned}
$$

\section{Point-coupling models}

For NLPC models, the incompressibility reads

$$
\begin{aligned}
K_{\mathrm{NLPC}}= & 9\left(\frac{\partial P_{\mathrm{NLPC}}}{\partial \rho}\right)_{y=1 / 2}=9\left[\alpha_{\mathrm{V}} \rho+3 \gamma_{\mathrm{V}} \rho^{3}+\frac{k_{F}^{2}}{3\left(k_{F}^{2}+M^{* 2}\right)^{1 / 2}}+\frac{\rho M^{*}}{\left(k_{F}^{2}+M^{* 2}\right)^{1 / 2}} \frac{\partial M^{*}}{\partial \rho}\right. \\
& \left.+2\left(\eta_{1}+\eta_{2} \rho_{s}\right) \rho_{s} \rho+2\left(\eta_{1}+2 \eta_{2} \rho_{s}\right) \rho^{2} \frac{\partial \rho_{s}}{\partial \rho}\right],
\end{aligned}
$$

where

$$
\begin{aligned}
\frac{\partial M^{*}}{\partial \rho} & =\frac{2\left(\eta_{1}+2 \eta_{2} \rho_{s}\right) \rho+\frac{M^{*}}{E_{F}^{*}}\left(\alpha_{s}+2 \beta_{s} \rho_{s}+3 \gamma_{s} \rho_{s}^{2}+2 \eta_{2} \rho^{2}\right)}{1-3\left(\alpha_{s}+2 \beta_{s} \rho_{s}+3 \gamma_{s} \rho_{s}^{2}+2 \eta_{2} \rho^{2}\right)\left(\frac{\rho_{s}}{M^{*}}-\frac{\rho}{E_{F}^{*}}\right)}, \\
\frac{\partial \rho_{s}}{\partial \rho} & =\frac{M^{*}}{\left(k_{F}^{2}+M^{* 2}\right)^{1 / 2}}+3\left(\frac{\rho_{s}}{M^{*}}-\frac{\rho}{E_{F}^{*}}\right) \frac{\partial M^{*}}{\partial \rho} .
\end{aligned}
$$


The skewness coefficient is

$$
\begin{aligned}
Q_{\mathrm{NLPC}} & =\left.27 \rho^{3} \frac{\partial^{3}\left(\mathcal{E}_{\mathrm{NLPC}} / \rho\right)}{\partial \rho^{3}}\right|_{y=1 / 2} \\
& =27 \rho^{3}\left[\frac{1}{\rho} \frac{\partial^{3} \mathcal{E}_{\mathrm{NLPC}}}{\partial \rho^{3}}-\frac{3}{\rho^{2}} \frac{\partial^{2} \mathcal{E}_{\mathrm{NLPC}}}{\partial \rho^{2}}+\frac{6}{\rho^{3}} \frac{\partial \mathcal{E}_{\mathrm{NLPC}}}{\partial \rho}-\frac{6 \mathcal{E}_{\mathrm{NLPC}}}{\rho^{4}}\right]_{y=1 / 2},
\end{aligned}
$$

with

$$
\begin{aligned}
\frac{\partial \mathcal{E}_{\mathrm{NLPC}}}{\partial \rho}= & \left(k_{F}^{2}+M^{* 2}\right)^{1 / 2}+\alpha_{\mathrm{V}} \rho+\gamma_{\mathrm{V}} \rho^{3}+2\left(\eta_{1}+\eta_{2} \rho_{s}\right) \rho_{s} \rho \\
\frac{\partial^{2} \mathcal{E}_{\mathrm{NLPC}}}{\partial \rho^{2}}= & \frac{1}{2 E_{F}^{*}}\left(\frac{\pi^{2}}{k_{F}}+2 M^{*} \frac{\partial M^{*}}{\partial \rho}\right)+\alpha_{\mathrm{V}}+3 \gamma_{\mathrm{V}} \rho^{2}+2\left(\eta_{1}+2 \eta_{2} \rho_{s}\right) \rho \frac{\partial \rho_{s}}{\partial \rho} \\
& +2\left(\eta_{1}+\eta_{2} \rho_{s}\right) \rho_{s}, \quad \text { and } \\
\frac{\partial^{3} \mathcal{E}_{\mathrm{NLPC}}}{\partial \rho^{3}}= & -\frac{1}{4 E_{F}^{* 3}}\left(\frac{\pi^{2}}{k_{F}}+2 M^{*} \frac{\partial M^{*}}{\partial \rho}\right)^{2}+\frac{1}{2 E_{F}^{*}}\left[-\frac{\pi^{4}}{2 k_{F}^{4}}+2\left(\frac{\partial M^{*}}{\partial \rho}\right)^{2}+2 M^{*} \frac{\partial^{2} M^{*}}{\partial \rho^{2}}\right] \\
& +6 \gamma_{\mathrm{V}} \rho+4\left(\eta_{1}+2 \eta_{2} \rho_{s}\right) \frac{\partial \rho_{s}}{\partial \rho}+4 \eta_{2} \rho\left(\frac{\partial \rho_{s}}{\partial \rho}\right)^{2}+2\left(\eta_{1}+2 \eta_{2} \rho_{s}\right) \rho \frac{\partial^{2} \rho_{s}}{\partial \rho^{2}} .
\end{aligned}
$$

The slope and curvature of $\mathcal{S}_{\mathrm{NLPC}}$ are

$$
\begin{aligned}
L_{\mathrm{NLPC}} & =3 \rho\left(\frac{\partial \mathcal{S}_{\mathrm{NLPC}}}{\partial \rho}\right) \\
& =\frac{k_{F}^{2}}{3 E_{F}^{*}}-\frac{k_{F}^{4}}{6 E_{F}^{* 3}}\left(1+\frac{2 M^{*} k_{F}}{\pi^{2}} \frac{\partial M^{*}}{\partial \rho}\right)+\frac{3}{2} \alpha_{\mathrm{TV}} \rho+3 \eta_{3} \rho_{s} \rho+3 \eta_{3} \rho^{2} \frac{\partial \rho_{s}}{\partial \rho}
\end{aligned}
$$

and

$$
\begin{aligned}
K_{\mathrm{sym}}^{\mathrm{NLPC}}= & 9 \rho^{2}\left(\frac{\partial^{2} \mathcal{S}_{\mathrm{NLPC}}}{\partial \rho^{2}}\right)=9 \rho^{2}\left\{-\frac{\pi^{2}}{12 E_{F}^{* 3} k_{F}}\left(\frac{\pi^{2}}{k_{F}}+2 M^{*} \frac{\partial M^{*}}{\partial \rho}\right)-\frac{\pi^{4}}{12 E_{F}^{*} k_{F}^{4}}+2 \eta_{3} \frac{\partial \rho_{s}}{\partial \rho}\right. \\
& -\left[\frac{\pi^{4}}{24 E_{F}^{* 3} k_{F}^{2}}-\frac{k_{F} \pi^{2}}{8 E_{F}^{* 5}}\left(\frac{\pi^{2}}{k_{F}}+2 M^{*} \frac{\partial M^{*}}{\partial \rho}\right)\right]\left(1+\frac{2 M^{*} k_{F}}{\pi^{2}} \frac{\partial M^{*}}{\partial \rho}\right)+\eta_{3} \rho \frac{\partial^{2} \rho_{s}}{\partial \rho^{2}} \\
& \left.-\frac{k_{F} \pi^{2}}{12 E_{F}^{* 3}}\left[\frac{M^{*}}{k_{F}^{2}} \frac{\partial M^{*}}{\partial \rho}+\frac{2 k_{F}}{\pi^{2}}\left(\frac{\partial M^{*}}{\partial \rho}\right)^{2}+\frac{2 k_{F} M^{*}}{\pi^{2}} \frac{\partial^{2} M^{*}}{\partial \rho^{2}}\right]\right\} .
\end{aligned}
$$

The chemical potentials for any proton fraction are

$$
\begin{aligned}
\mu_{p}^{\mathrm{NLPC}} & =\frac{\partial \mathcal{E}_{\mathrm{NLPC}}}{\partial \rho_{p}} \\
& =\left(k_{F}^{2}+M^{* 2}\right)^{1 / 2}+\alpha_{\mathrm{V}} \rho+\gamma_{\mathrm{V}} \rho^{3}+\alpha_{\mathrm{TV}} \rho_{3}+\gamma_{\mathrm{TV}} \rho_{3}^{3}+2 \eta_{1} \rho_{s} \rho+2 \eta_{2} \rho_{s}^{2} \rho+2 \eta_{3} \rho_{s} \rho_{3}, \\
\mu_{n}^{\mathrm{NLPC}} & =\frac{\partial \mathcal{E}_{\mathrm{NLPC}}}{\partial \rho_{n}} \\
& =\left(k_{F}^{2}+M^{* 2}\right)^{1 / 2}+\alpha_{\mathrm{V}} \rho+\gamma_{\mathrm{V}} \rho^{3}-\alpha_{\mathrm{TV}} \rho_{3}-\gamma_{\mathrm{TV}} \rho_{3}^{3}+2 \eta_{1} \rho_{s} \rho+2 \eta_{2} \rho_{s}^{2} \rho-2 \eta_{3} \rho_{s} \rho_{3} .
\end{aligned}
$$




\section{APPENDIX B: SATURATION PROPERTIES}

TABLE VII. Saturation properties of all RMF models used in this work. All quantities are given in MeV, except for the dimensionless effective mass, $m^{*}=M^{*} / M$, and the saturation density $\rho_{0}$, given in $\mathrm{fm}^{-3}$. Here $K^{\prime}=-Q_{0}$.

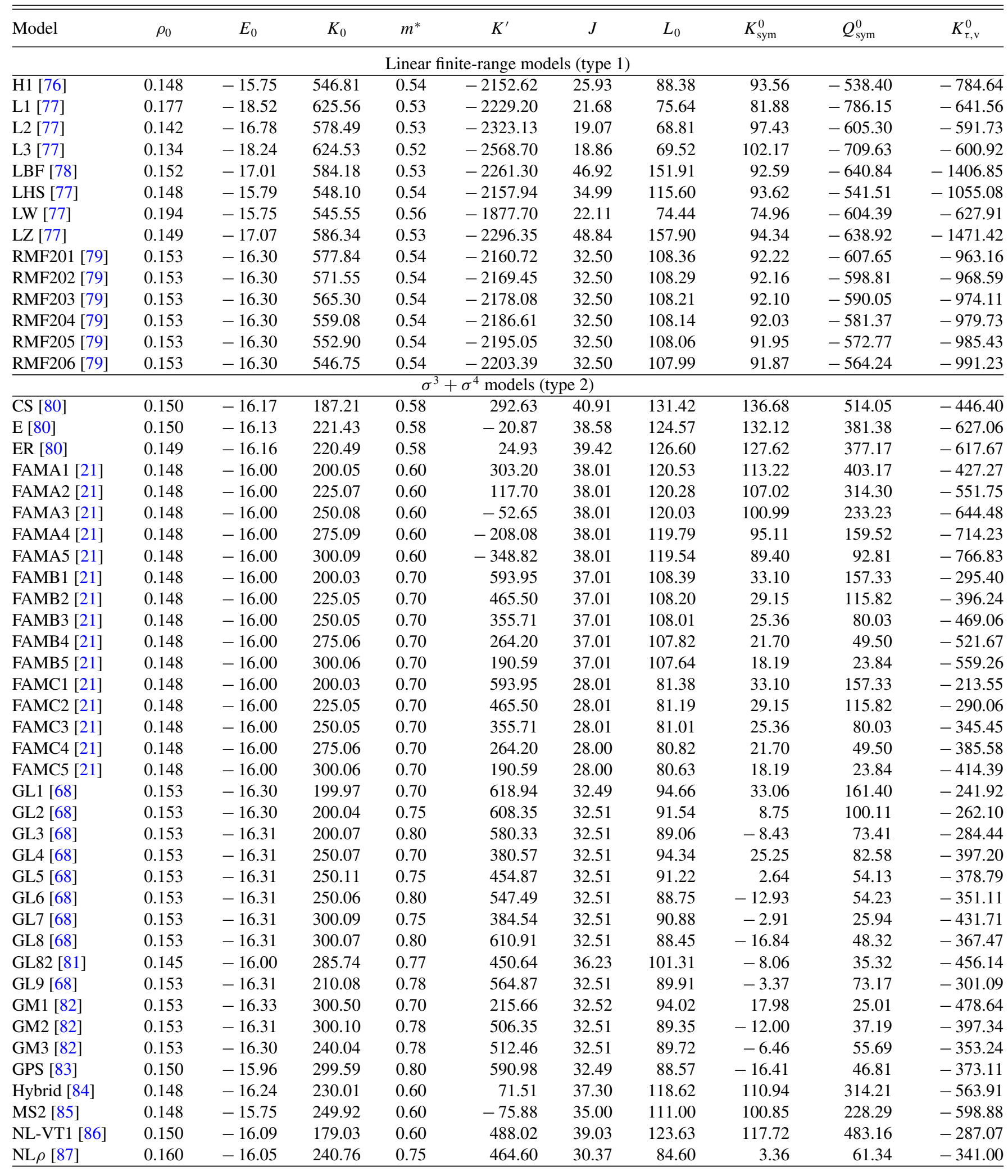


TABLE VII. (Continued.)

\begin{tabular}{|c|c|c|c|c|c|c|c|c|c|c|}
\hline Model & $\rho_{0}$ & $E_{0}$ & $K_{0}$ & $m^{*}$ & $K^{\prime}$ & $J$ & $L_{0}$ & $K_{\mathrm{sym}}^{0}$ & $Q_{\mathrm{sym}}^{0}$ & $K_{\tau, \mathrm{v}}^{0}$ \\
\hline NL06 [78] & 0.147 & -16.05 & 195.09 & 0.60 & 367.03 & 39.33 & 124.14 & 110.85 & 412.35 & -400.47 \\
\hline NL065 [78] & 0.150 & -16.37 & 256.86 & 0.65 & 220.00 & 38.98 & 117.77 & 55.86 & 132.74 & -549.91 \\
\hline NL075 [78] & 0.151 & -16.64 & 281.12 & 0.75 & 418.96 & 38.96 & 110.44 & -0.54 & 35.98 & -498.61 \\
\hline NL1 [77] & 0.152 & -16.42 & 211.09 & 0.57 & 32.69 & 43.46 & 140.07 & 142.68 & 444.67 & -676.04 \\
\hline NL1J4 [88] & 0.152 & -16.42 & 211.70 & 0.57 & -3.91 & 40.00 & 130.06 & 146.26 & 448.71 & -636.53 \\
\hline NL2 [77] & 0.146 & -17.03 & 399.37 & 0.67 & -68.42 & 43.86 & 129.66 & 20.10 & -51.87 & -780.04 \\
\hline NL3 [89] & 0.148 & -16.24 & 271.53 & 0.60 & -202.91 & 37.40 & 118.53 & 100.88 & 181.31 & -698.85 \\
\hline NL3-II [89] & 0.149 & -16.26 & 271.72 & 0.59 & -222.71 & 37.70 & 119.71 & 103.43 & 183.75 & -712.92 \\
\hline NL3* [90] & 0.150 & -16.31 & 258.25 & 0.59 & -122.04 & 38.68 & 122.63 & 105.56 & 223.95 & -688.19 \\
\hline NL4 [91] & 0.148 & -16.16 & 270.34 & 0.60 & -193.75 & 36.24 & 114.92 & 99.72 & 180.84 & -672.14 \\
\hline NLB [92] & 0.148 & -15.77 & 421.02 & 0.61 & -727.93 & 35.01 & 108.26 & 54.94 & -132.35 & -781.79 \\
\hline NLM [88] & 0.160 & -16.00 & 200.00 & 0.70 & 600.49 & 30.00 & 87.02 & 33.20 & 161.25 & -227.65 \\
\hline NLM2 [88] & 0.160 & -17.00 & 200.00 & 0.70 & 675.54 & 30.00 & 86.95 & 33.27 & 170.92 & -194.74 \\
\hline NLM3 [88] & 0.145 & -16.00 & 200.00 & 0.70 & 591.82 & 30.00 & 87.45 & 33.01 & 156.01 & -232.93 \\
\hline NLM4 [88] & 0.160 & -16.00 & 300.00 & 0.70 & 196.02 & 30.00 & 86.25 & 17.69 & 22.57 & -443.46 \\
\hline NLM5 [88] & 0.160 & -16.00 & 200.00 & 0.55 & -216.98 & 30.00 & 103.18 & 179.44 & 524.64 & -551.60 \\
\hline NLM6 [88] & 0.160 & -16.00 & 200.00 & 0.70 & 600.49 & 40.00 & 117.02 & 33.20 & 161.24 & -317.57 \\
\hline NLRA [94] & 0.157 & -16.25 & 320.48 & 0.63 & -216.23 & 38.90 & 119.09 & 62.11 & 26.63 & -732.77 \\
\hline NLRA1 [95] & 0.147 & -16.15 & 285.23 & 0.60 & -279.28 & 36.45 & 115.38 & 95.72 & 139.44 & -709.55 \\
\hline NLS [96] & 0.150 & -16.44 & 262.94 & 0.60 & -57.53 & 42.07 & 131.59 & 94.22 & 195.27 & -724.10 \\
\hline NLSH [89] & 0.146 & -16.36 & 355.65 & 0.60 & -602.90 & 36.13 & 113.68 & 79.83 & -23.79 & -795.00 \\
\hline NLZ [86] & 0.151 & -16.18 & 172.84 & 0.58 & 422.59 & 41.72 & 133.91 & 140.19 & 577.02 & -335.86 \\
\hline RMF302 [79] & 0.153 & -16.30 & 249.71 & 0.78 & 502.35 & 32.50 & 89.66 & -7.35 & 51.33 & -364.94 \\
\hline RMF303 [79] & 0.153 & -16.30 & 248.88 & 0.78 & 504.91 & 32.50 & 89.62 & -7.57 & 51.73 & -363.46 \\
\hline RMF304 [79] & 0.153 & -16.30 & 248.04 & 0.78 & 507.43 & 32.50 & 89.57 & -7.78 & 52.13 & -361.98 \\
\hline RMF305 [79] & 0.153 & -16.30 & 246.37 & 0.78 & 512.37 & 32.50 & 89.49 & -8.21 & 52.92 & -359.04 \\
\hline RMF306 [79] & 0.153 & -16.30 & 244.69 & 0.79 & 517.18 & 32.50 & 89.41 & -8.63 & 53.72 & -356.09 \\
\hline RMF307 [79] & 0.153 & -16.30 & 243.84 & 0.79 & 519.54 & 32.50 & 89.37 & -8.84 & 54.11 & -354.63 \\
\hline RMF308 [79] & 0.153 & -16.30 & 242.99 & 0.79 & 521.85 & 32.50 & 89.32 & -9.04 & 54.50 & -353.15 \\
\hline RMF309 [79] & 0.153 & -16.30 & 241.30 & 0.79 & 526.40 & 32.50 & 89.24 & -9.45 & 55.28 & -350.22 \\
\hline RMF310 [79] & 0.153 & -16.30 & 238.75 & 0.79 & 532.98 & 32.50 & 89.12 & -10.04 & 56.45 & -345.82 \\
\hline RMF311 [79] & 0.153 & -16.30 & 237.89 & 0.79 & 535.10 & 32.50 & 89.08 & -10.24 & 56.83 & -344.35 \\
\hline RMF312 [79] & 0.153 & -16.30 & 237.03 & 0.79 & 537.20 & 32.50 & 89.04 & -10.44 & 57.22 & -342.89 \\
\hline RMF313 [79] & 0.153 & -16.30 & 235.31 & 0.80 & 541.28 & 32.50 & 88.96 & -10.82 & 57.98 & -339.96 \\
\hline RMF314 [79] & 0.153 & -16.30 & 234.43 & 0.80 & 543.28 & 32.50 & 88.92 & -11.01 & 58.36 & -338.47 \\
\hline RMF315 [79] & 0.153 & -16.30 & 234.01 & 0.80 & 544.27 & 32.50 & 88.91 & -11.10 & 58.55 & -337.76 \\
\hline RMF316 [79] & 0.153 & -16.30 & 233.57 & 0.80 & 545.22 & 32.50 & 88.89 & -11.20 & 58.74 & -337.02 \\
\hline RMF317 [79] & 0.153 & -16.30 & 232.70 & 0.80 & 547.17 & 32.50 & 88.85 & -11.38 & 59.12 & -335.55 \\
\hline RMF401 [79] & 0.153 & -16.30 & 229.99 & 0.71 & 477.86 & 32.50 & 93.79 & 23.04 & 100.51 & -344.81 \\
\hline RMF402 [79] & 0.153 & -16.30 & 231.99 & 0.71 & 469.28 & 32.50 & 93.77 & 22.74 & 97.75 & -350.20 \\
\hline RMF403 [79] & 0.153 & -16.30 & 229.99 & 0.72 & 486.57 & 32.50 & 93.13 & 18.06 & 90.88 & -343.67 \\
\hline RMF404 [79] & 0.153 & -16.30 & 231.99 & 0.72 & 478.64 & 32.50 & 93.11 & 17.78 & 88.39 & -348.78 \\
\hline RMF405 [79] & 0.153 & -16.30 & 233.99 & 0.72 & 470.83 & 32.50 & 93.10 & 17.50 & 85.94 & -353.76 \\
\hline
\end{tabular}


TABLE VII. (Continued.)

\begin{tabular}{|c|c|c|c|c|c|c|c|c|c|c|}
\hline Model & $\rho_{0}$ & $E_{0}$ & $K_{0}$ & $m^{*}$ & $K^{\prime}$ & $J$ & $L_{0}$ & $K_{\mathrm{sym}}^{0}$ & $Q_{\mathrm{sym}}^{0}$ & $K_{\tau, \mathrm{v}}^{0}$ \\
\hline RMF406 [79] & 0.153 & -16.30 & 233.99 & 0.78 & 520.06 & 32.50 & 89.75 & -5.80 & 58.72 & -344.85 \\
\hline RMF407 [79] & 0.153 & -16.30 & 229.99 & 0.73 & 493.83 & 32.50 & 92.50 & 13.42 & 82.67 & -342.96 \\
\hline RMF409 [79] & 0.153 & -16.30 & 233.99 & 0.73 & 479.45 & 32.50 & 92.47 & 12.88 & 78.25 & -352.46 \\
\hline RMF410 [79] & 0.153 & -16.30 & 235.99 & 0.73 & 472.46 & 32.50 & 92.46 & 12.62 & 76.09 & -357.02 \\
\hline RMF411 [79] & 0.153 & -16.30 & 229.99 & 0.74 & 500.11 & 32.50 & 91.90 & 9.09 & 75.82 & -342.47 \\
\hline RMF413 [79] & 0.153 & -16.30 & 233.99 & 0.74 & 487.20 & 32.50 & 91.87 & 8.58 & 71.90 & -351.36 \\
\hline RMF414 [79] & 0.153 & -16.30 & 235.99 & 0.74 & 480.94 & 32.50 & 91.86 & 8.33 & 69.99 & -355.62 \\
\hline RMF415 [79] & 0.153 & -16.30 & 229.98 & 0.75 & 505.92 & 32.50 & 91.33 & 5.06 & 70.28 & -342.01 \\
\hline RMF416 [79] & 0.153 & -16.30 & 231.98 & 0.75 & 500.17 & 32.50 & 91.32 & 4.82 & 68.54 & -346.19 \\
\hline RMF417 [79] & 0.153 & -16.30 & 233.99 & 0.75 & 494.57 & 32.50 & 91.30 & 4.58 & 66.84 & -350.26 \\
\hline RMF418 [79] & 0.153 & -16.30 & 235.98 & 0.75 & 489.09 & 32.50 & 91.29 & 4.34 & 65.16 & -354.20 \\
\hline RMF423 [79] & 0.153 & -16.30 & 231.99 & 0.77 & 514.26 & 32.50 & 90.26 & -2.38 & 61.57 & -343.86 \\
\hline RMF424 [79] & 0.153 & -16.30 & 245.99 & 0.79 & 523.99 & 32.50 & 89.21 & -9.88 & 53.59 & -355.12 \\
\hline RMF425 [79] & 0.153 & -16.30 & 247.99 & 0.79 & 523.21 & 32.50 & 89.20 & -10.06 & 52.90 & -357.07 \\
\hline RMF426 [79] & 0.153 & -16.30 & 249.99 & 0.79 & 522.57 & 32.50 & 89.19 & -10.24 & 52.23 & -358.93 \\
\hline RMF427 [79] & 0.153 & -16.30 & 235.98 & 0.80 & 546.20 & 32.50 & 88.83 & -11.67 & 58.05 & -339.04 \\
\hline RMF428 [79] & 0.153 & -16.30 & 237.98 & 0.80 & 545.82 & 32.50 & 88.81 & -11.85 & 57.43 & -341.04 \\
\hline RMF429 [79] & 0.153 & -16.30 & 239.99 & 0.80 & 545.60 & 32.50 & 88.80 & -12.02 & 56.84 & -342.95 \\
\hline RMF430 [79] & 0.153 & -16.30 & 241.99 & 0.80 & 545.53 & 32.50 & 88.79 & -12.19 & 56.26 & -344.77 \\
\hline RMF431 [79] & 0.153 & -16.30 & 243.98 & 0.80 & 545.62 & 32.50 & 88.78 & -12.36 & 55.71 & -346.50 \\
\hline RMF432 [79] & 0.153 & -16.30 & 245.98 & 0.80 & 545.86 & 32.50 & 88.77 & -12.53 & 55.19 & -348.16 \\
\hline RMF433 [79] & 0.153 & -16.30 & 247.99 & 0.80 & 546.26 & 32.50 & 88.76 & -12.70 & 54.68 & -349.73 \\
\hline VT [80] & 0.153 & -16.09 & 172.74 & 0.59 & 482.84 & 39.72 & 126.83 & 130.05 & 542.92 & -276.41 \\
\hline \multicolumn{11}{|c|}{$\sigma^{3}+\sigma^{4}+\omega_{0}^{4}$ models (type 3) } \\
\hline$\overline{\mathrm{BM}-\mathrm{A}[101]}$ & 0.179 & -15.17 & 188.32 & 0.61 & 436.32 & 19.62 & 51.88 & -18.05 & -36.02 & -209.14 \\
\hline BM-B [101] & 0.156 & -13.47 & 170.77 & 0.64 & 504.54 & 17.42 & 45.46 & -15.61 & -5.33 & -154.06 \\
\hline BM-C [101] & 0.142 & -12.36 & 163.10 & 0.65 & 547.19 & 16.01 & 41.49 & -14.20 & 7.05 & -123.94 \\
\hline DJM [101] & 0.172 & -14.81 & 244.73 & 0.57 & -147.54 & 20.21 & 62.95 & 32.66 & -302.43 & -383.00 \\
\hline DJM-C [101] & 0.181 & -15.67 & 329.44 & 0.54 & -229.30 & 21.89 & 68.46 & 17.17 & -468.73 & -441.27 \\
\hline EMFT1 [99] & 0.159 & -13.56 & 166.12 & 0.59 & -3.47 & 18.60 & 56.58 & 30.48 & -165.49 & -310.17 \\
\hline HD [102] & 0.177 & -16.22 & 284.18 & 0.67 & 101.75 & 35.69 & 105.93 & 44.58 & 93.60 & -553.09 \\
\hline LB [102] & 0.184 & -15.26 & 316.76 & 0.59 & 51.67 & 31.92 & 96.73 & 27.18 & -268.79 & -537.44 \\
\hline MB [102] & 0.190 & -15.07 & 341.71 & 0.59 & 1375.71 & 32.49 & 89.97 & -28.08 & -32.74 & -205.68 \\
\hline MS1 [103] & 0.148 & -15.75 & 249.97 & 0.60 & 161.37 & 35.00 & 106.75 & 38.56 & -150.95 & -533.03 \\
\hline MS3 [104] & 0.148 & -15.75 & 249.98 & 0.60 & 563.90 & 35.00 & 102.38 & -0.20 & -111.15 & -383.52 \\
\hline NLSV1 [105] & 0.149 & -16.26 & 270.15 & 0.61 & 74.63 & 37.30 & 114.68 & 58.97 & -27.80 & -597.46 \\
\hline NLSV2 [105] & 0.147 & -16.23 & 291.65 & 0.62 & 174.88 & 36.76 & 111.52 & 39.50 & -100.07 & -562.74 \\
\hline PK1 [106] & 0.148 & -16.27 & 282.69 & 0.61 & 27.82 & 37.64 & 115.88 & 55.30 & -87.79 & -628.58 \\
\hline TM1 [107] & 0.145 & -16.26 & 281.16 & 0.63 & 285.22 & 36.89 & 110.79 & 33.62 & -66.54 & -518.75 \\
\hline TM2 [107] & 0.132 & -16.16 & 343.82 & 0.57 & -265.60 & 35.98 & 113.03 & 56.07 & -237.87 & -709.40 \\
\hline TMA [108] & 0.147 & -16.02 & 318.15 & 0.63 & 572.12 & 30.66 & 90.14 & 10.75 & -108.74 & -367.99 \\
\hline Z271 [17] & 0.148 & -16.24 & 270.99 & 0.80 & 733.54 & 35.92 & 98.86 & -16.40 & 49.00 & -341.94 \\
\hline
\end{tabular}


TABLE VII. (Continued.)

\begin{tabular}{|c|c|c|c|c|c|c|c|c|c|c|}
\hline Model & $\rho_{0}$ & $E_{0}$ & $K_{0}$ & $m^{*}$ & $K^{\prime}$ & $J$ & $L_{0}$ & $K_{\mathrm{sym}}^{0}$ & $Q_{\mathrm{sym}}^{0}$ & $K_{\tau, \mathrm{v}}^{0}$ \\
\hline \multicolumn{11}{|c|}{$\sigma^{3}+\sigma^{4}+\omega^{4}+$ cross-terms models (type 4 ) } \\
\hline$\overline{\text { BKA20 [67] }}$ & 0.146 & -15.93 & 237.95 & 0.64 & 464.66 & 32.24 & 75.38 & -15.04 & 198.38 & -320.11 \\
\hline BKA24 [67] & 0.147 & -15.95 & 227.06 & 0.60 & 273.58 & 34.19 & 84.80 & -14.95 & 112.40 & -421.55 \\
\hline BSR1 [109] & 0.148 & -16.02 & 239.89 & 0.61 & 35.68 & 31.04 & 59.41 & 12.96 & 468.10 & -334.65 \\
\hline BSR2 [109] & 0.149 & -16.03 & 239.93 & 0.61 & 48.06 & 31.50 & 62.02 & -3.14 & 403.21 & -362.81 \\
\hline BSR4 [109] & 0.150 & -16.08 & 238.57 & 0.61 & -4.00 & 33.17 & 73.23 & -20.71 & 420.06 & -461.34 \\
\hline BSR5 [109] & 0.151 & -16.12 & 235.71 & 0.61 & 10.96 & 34.46 & 83.37 & -14.16 & 346.84 & -510.53 \\
\hline BSR6 [109] & 0.149 & -16.13 & 235.75 & 0.60 & 7.59 & 35.62 & 85.68 & -49.55 & 352.00 & -560.86 \\
\hline BSR7 [109] & 0.149 & -16.18 & 231.80 & 0.60 & 19.80 & 37.26 & 99.14 & -16.97 & 198.47 & -603.32 \\
\hline BSR8 [109] & 0.147 & -16.04 & 230.95 & 0.61 & 290.85 & 31.08 & 60.25 & -0.74 & 238.23 & -286.36 \\
\hline BSR9 [109] & 0.147 & -16.07 & 232.50 & 0.60 & 297.11 & 31.61 & 63.89 & -11.32 & 202.86 & -313.03 \\
\hline BSR14 [109] & 0.147 & -16.18 & 235.47 & 0.61 & 317.10 & 36.32 & 93.85 & -41.95 & 112.53 & -478.66 \\
\hline BSR15 [109] & 0.146 & -16.03 & 226.82 & 0.61 & 512.29 & 30.97 & 61.79 & -21.36 & 128.26 & -252.5 \\
\hline BSR16 [109] & 0.146 & -16.05 & 224.98 & 0.61 & 503.17 & 31.24 & 62.33 & -24.17 & 152.29 & -258.75 \\
\hline BSR17 [109] & 0.146 & -16.05 & 221.67 & 0.61 & 489.45 & 31.98 & 67.44 & -31.58 & 176.65 & -287.31 \\
\hline BSR18 [109] & 0.146 & -16.05 & 221.13 & 0.61 & 485.73 & 32.74 & 72.65 & -42.24 & 199.39 & -318.55 \\
\hline BSR19 [109] & 0.147 & -16.08 & 220.83 & 0.61 & 484.25 & 33.78 & 79.47 & -50.13 & 194.70 & -352.70 \\
\hline BSR20 [109] & 0.146 & -16.09 & 223.25 & 0.61 & 507.75 & 34.54 & 88.03 & -39.90 & 82.74 & -367.86 \\
\hline BSR21 [109] & 0.145 & -16.12 & 220.32 & 0.60 & 468.20 & 35.96 & 92.94 & -46.01 & 67.45 & -406.16 \\
\hline C1 [98] & 0.146 & -16.19 & 303.97 & 0.66 & 132.68 & 32.03 & 94.62 & 26.60 & -11.72 & -499.83 \\
\hline FSU-I [110] & 0.148 & -16.28 & 229.54 & 0.61 & 523.95 & 37.42 & 109.62 & 2.64 & -101.71 & -404.87 \\
\hline FSU-II [110] & 0.148 & -16.28 & 229.54 & 0.61 & 523.95 & 35.49 & 87.39 & -68.37 & 156.81 & -393.23 \\
\hline FSUGZ03 [113] & 0.147 & -16.07 & 232.48 & 0.60 & 297.13 & 31.54 & 63.98 & -11.66 & 203.43 & -313.79 \\
\hline FSUGZ06 [113] & 0.146 & -16.05 & 225.06 & 0.61 & 503.17 & 31.18 & 62.42 & -24.49 & 153.31 & -259.47 \\
\hline G1 [98] & 0.153 & -16.14 & 214.83 & 0.63 & 361.91 & 38.48 & 123.19 & 96.87 & 95.59 & -434.73 \\
\hline G2 [98] & 0.154 & -16.07 & 214.77 & 0.66 & 438.68 & 36.39 & 100.67 & -7.48 & 47.62 & -405.85 \\
\hline $\mathrm{G} 2 *[114]$ & 0.154 & -16.07 & 214.77 & 0.66 & 438.68 & 30.39 & 69.68 & -21.93 & 197.52 & $-297.6 C$ \\
\hline $\mathrm{HC}$ [102] & 0.169 & -15.75 & 231.91 & 0.68 & 390.71 & 31.01 & 58.53 & -99.04 & 933.54 & -351.62 \\
\hline IU-FSU [115] & 0.155 & -16.40 & 231.33 & 0.61 & 290.28 & 31.30 & 47.21 & 28.53 & 370.71 & -195.46 \\
\hline LA [102] & 0.179 & -15.46 & 301.59 & 0.60 & 145.52 & 33.48 & 78.67 & -61.77 & 264.62 & -495.82 \\
\hline MA [102] & 0.179 & -15.93 & 347.40 & 0.61 & 1279.91 & 32.39 & 71.47 & -74.53 & 265.76 & -240.0 \\
\hline NL3v1 [116] & 0.148 & -16.24 & 271.59 & 0.59 & -204.06 & 36.07 & 101.25 & 0.69 & 129.02 & -682.91 \\
\hline NL3v2 [116] & 0.148 & -16.24 & 271.59 & 0.59 & -204.06 & 34.98 & 87.77 & -46.15 & 442.19 & $-638.6 C$ \\
\hline NL3v3 [116] & 0.148 & -16.24 & 271.59 & 0.59 & -204.06 & 34.48 & 82.08 & -56.12 & 632.86 & -610.24 \\
\hline NL3v4 [116] & 0.148 & -16.24 & 271.59 & 0.59 & -204.06 & 34.02 & 76.96 & -59.87 & 819.51 & -579.46 \\
\hline NL3v5 [116] & 0.148 & -16.24 & 271.59 & 0.59 & -204.06 & 33.16 & 68.24 & -52.95 & 1128.57 & -513.65 \\
\hline NL3v6 [116] & 0.148 & -16.24 & 271.59 & 0.59 & -204.06 & 32.39 & 61.14 & -33.68 & 1323.38 & -446.44 \\
\hline S271v1 [116] & 0.148 & -16.24 & 271.00 & 0.70 & 295.64 & 35.73 & 95.93 & -44.06 & -53.63 & -514.99 \\
\hline S271v2 [116] & 0.148 & -16.24 & 271.00 & 0.70 & 295.64 & 35.06 & 86.87 & -90.33 & 14.94 & -516.79 \\
\hline S271v3 [116] & 0.148 & -16.24 & 271.00 & 0.70 & 295.64 & 34.42 & 78.86 & -121.00 & 180.94 & -508.12 \\
\hline
\end{tabular}


TABLE VII. (Continued.)

\begin{tabular}{|c|c|c|c|c|c|c|c|c|c|c|}
\hline Model & $\rho_{0}$ & $E_{0}$ & $K_{0}$ & $m^{*}$ & $K^{\prime}$ & $J$ & $L_{0}$ & $K_{\mathrm{sym}}^{0}$ & $Q_{\mathrm{sym}}^{0}$ & $K_{\tau, \mathrm{v}}^{0}$ \\
\hline S271v4 [116] & 0.148 & -16.24 & 271.00 & 0.70 & 295.64 & 33.83 & 71.76 & -139.52 & 390.88 & -491.78 \\
\hline S271v5 [116] & 0.148 & -16.24 & 271.00 & 0.70 & 295.64 & 33.27 & 65.44 & -148.63 & 609.90 & -469.88 \\
\hline SIG-OM [117] & 0.149 & -16.30 & 265.33 & 0.62 & 233.77 & 37.01 & 111.97 & 41.15 & -48.89 & -532.02 \\
\hline SVI-1 [118] & 0.149 & -16.30 & 263.90 & 0.62 & 490.57 & 37.05 & 116.54 & 95.84 & 208.24 & -386.76 \\
\hline SVI-2 [118] & 0.149 & -16.31 & 271.49 & 0.62 & 455.15 & 37.04 & 116.05 & 91.36 & 199.04 & -410.37 \\
\hline XS [104] & 0.148 & -16.30 & 229.98 & 0.60 & 702.85 & 31.83 & 54.97 & -28.80 & 128.26 & -190.62 \\
\hline $\mathrm{Z} 271 *[114]$ & 0.148 & -16.24 & 270.96 & 0.80 & 733.47 & 40.25 & 83.57 & -198.47 & 306.00 & -473.66 \\
\hline Z271s1 [116] & 0.148 & -16.24 & 271.00 & 0.80 & 733.59 & 34.95 & 86.86 & -64.86 & 144.67 & -350.90 \\
\hline Z271s2 [116] & 0.148 & -16.24 & 271.00 & 0.80 & 733.59 & 34.08 & 76.62 & -92.28 & 301.43 & -344.61 \\
\hline Z271s3 [116] & 0.148 & -16.24 & 271.00 & 0.80 & 733.59 & 33.27 & 67.81 & -104.58 & 457.51 & -327.87 \\
\hline Z271s4 [116] & 0.148 & -16.24 & 271.00 & 0.80 & 733.59 & 32.53 & 60.18 & -106.04 & 580.42 & -304.23 \\
\hline Z271v3 [116] & 0.148 & -16.24 & 271.00 & 0.80 & 733.59 & 34.54 & 80.23 & -120.39 & 57.82 & -384.59 \\
\hline Z271v4 [116] & 0.148 & -16.24 & 271.00 & 0.80 & 733.59 & 34.29 & 77.00 & -133.76 & 112.71 & -387.31 \\
\hline Z271v5 [116] & 0.148 & -16.24 & 271.00 & 0.80 & 733.59 & 34.04 & 73.90 & -145.15 & 177.12 & -388.52 \\
\hline Z271v6 [116] & 0.148 & -16.24 & 271.00 & 0.80 & 733.59 & 33.80 & 70.94 & -154.74 & 248.50 & -388.36 \\
\hline \multicolumn{11}{|c|}{ Density-dependent models (type 5) } \\
\hline$\overline{\mathrm{DD}}[120]$ & 0.149 & -16.02 & 239.99 & 0.56 & -134.65 & 31.64 & 55.98 & -95.30 & 576.86 & -462.57 \\
\hline DD-F [121] & 0.147 & -16.04 & 223.32 & 0.56 & 758.73 & 31.63 & 56.00 & -139.80 & 467.35 & -285.54 \\
\hline DD-ME1 [122] & 0.152 & -16.20 & 244.72 & 0.58 & -316.66 & 33.06 & 55.45 & -101.05 & 705.59 & -505.50 \\
\hline DD-ME2 [123] & 0.152 & -16.14 & 250.92 & 0.57 & -478.75 & 32.30 & 51.25 & -87.19 & 776.91 & -492.45 \\
\hline DD2 [124] & 0.149 & -16.02 & 242.72 & 0.56 & -168.65 & 31.67 & 55.04 & -93.23 & 598.138 & -461.69 \\
\hline PKDD [106] & 0.150 & -16.27 & 262.19 & 0.57 & 118.73 & 36.79 & 90.21 & -80.55 & 24.36 & -580.97 \\
\hline FZ3 [125] & 0.151 & -15.93 & 294.71 & 0.74 & 364.21 & 29.93 & 33.93 & -261.59 & 424.03 & -423.24 \\
\hline FZ4 [125] & 0.151 & -15.85 & 302.83 & 0.74 & 136.60 & 30.00 & 36.61 & -241.23 & 544.22 & -444.36 \\
\hline PC-F1 [126] & 0.151 & -16.17 & 254.57 & 0.61 & 289.97 & 37.75 & 117.04 & 74.55 & 78.70 & -494.39 \\
\hline PC-F3 [126] & 0.151 & -16.18 & 254.67 & 0.61 & 288.23 & 38.24 & 118.51 & 74.67 & 78.49 & -502.28 \\
\hline VA2 [125] & 0.149 & -15.90 & 285.10 & 0.60 & 805.10 & 34.89 & 125.69 & 116.53 & -376.72 & -282.67 \\
\hline VA3 [125] & 0.149 & -16.07 & 298.68 & 0.63 & 100.92 & 39.22 & 186.06 & 401.48 & -770.96 & -652.02 \\
\hline VA4 [125] & 0.151 & -16.11 & 322.41 & 0.66 & -668.03 & 34.56 & 125.32 & 190.20 & 50.99 & -821.39 \\
\hline VZ0 [125] & 0.145 & -16.29 & 563.38 & 0.54 & -2243.19 & 37.93 & 124.93 & 95.78 & -571.95 & -1151.19 \\
\hline VZ1 [125] & 0.148 & -15.86 & 379.55 & 0.70 & -288.93 & 32.72 & 95.68 & 23.21 & -9.55 & -623.69 \\
\hline VZ2 [125] & 0.149 & -15.99 & 324.20 & 0.60 & 435.61 & 34.79 & 127.33 & 133.57 & -390.54 & -459.32 \\
\hline VZ3 [125] & 0.150 & -16.04 & 297.70 & 0.62 & 75.77 & 34.60 & 123.95 & 154.81 & -195.99 & -557.32 \\
\hline VZ4 [125] & 0.151 & -15.90 & 305.48 & 0.65 & -286.33 & 32.70 & 97.38 & 62.70 & 335.81 & -612.88 \\
\hline \multicolumn{11}{|c|}{$\delta$ meson models (type 7 ) } \\
\hline$\overline{\mathrm{DD}-\mathrm{ME} \delta}[127]$ & 0.152 & -16.08 & 219.60 & 0.61 & 748.31 & 32.18 & 51.43 & -124.96 & 843.03 & -258.28 \\
\hline $\mathrm{DDH} \delta[128]$ & 0.153 & -16.25 & 240.18 & 0.55 & 539.59 & 25.34 & 45.33 & 52.91 & 783.21 & -117.22 \\
\hline GDFM [32] & 0.178 & -17.66 & 323.80 & 0.68 & -2783.75 & 33.05 & 67.89 & 75.08 & 491.06 & -915.98 \\
\hline $\mathrm{NL} \delta$ [129] & 0.160 & -16.00 & 240.17 & 0.75 & 463.63 & 30.60 & 101.46 & 117.03 & 227.51 & -295.86 \\
\hline NL3 $\delta[89]$ & 0.148 & -16.24 & 269.94 & 0.60 & -198.33 & 37.67 & 149.98 & 393.86 & 1484.30 & -616.21 \\
\hline
\end{tabular}




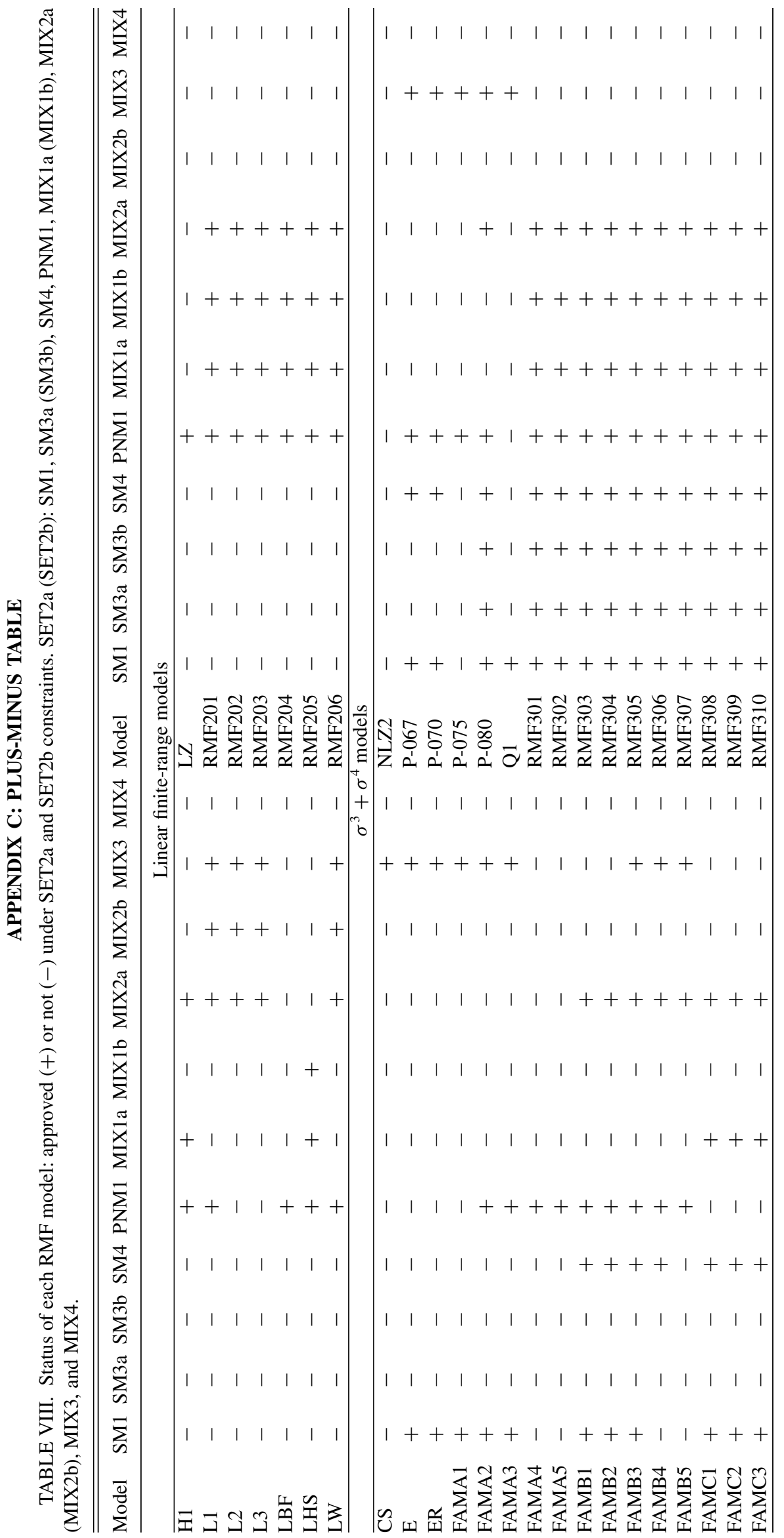




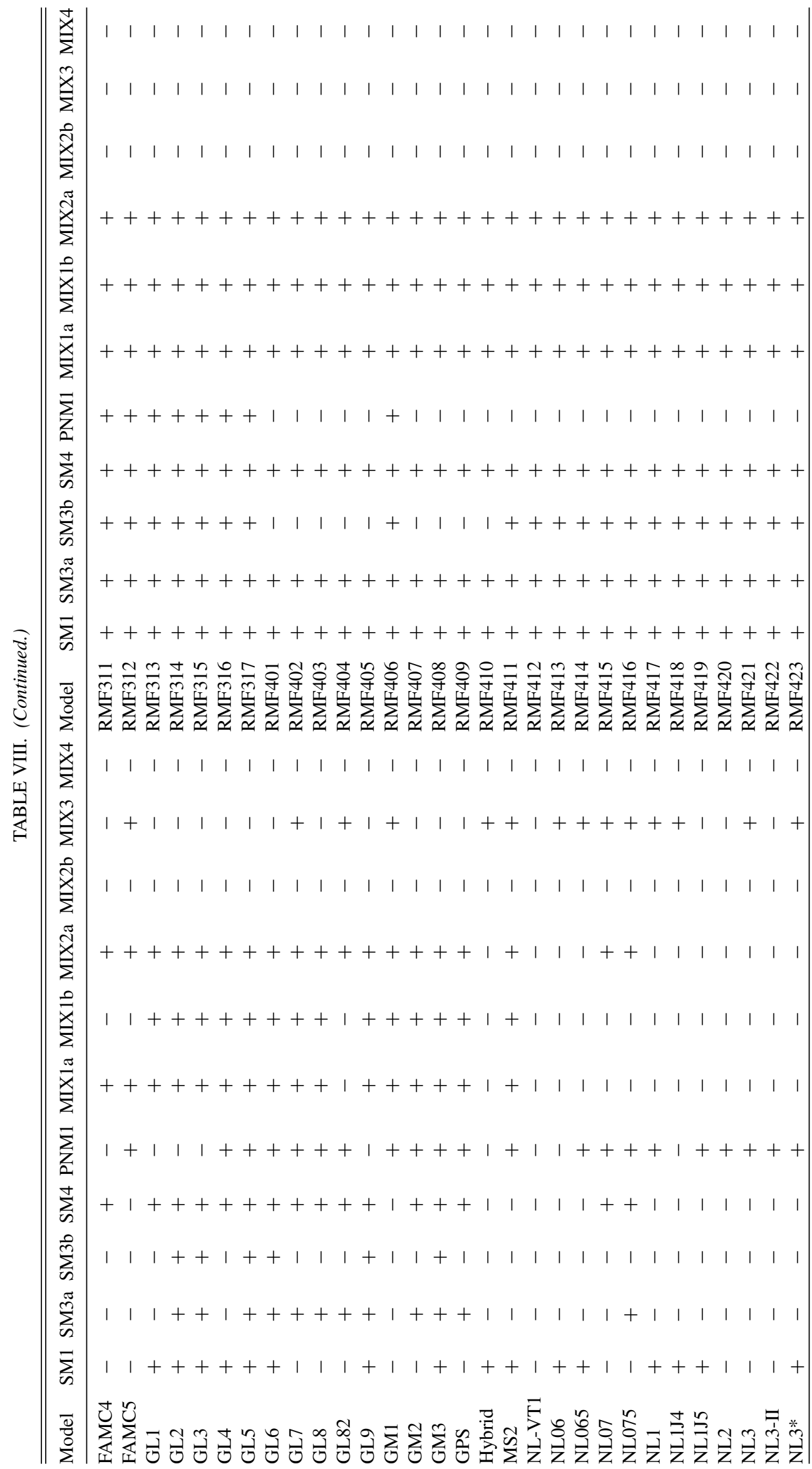




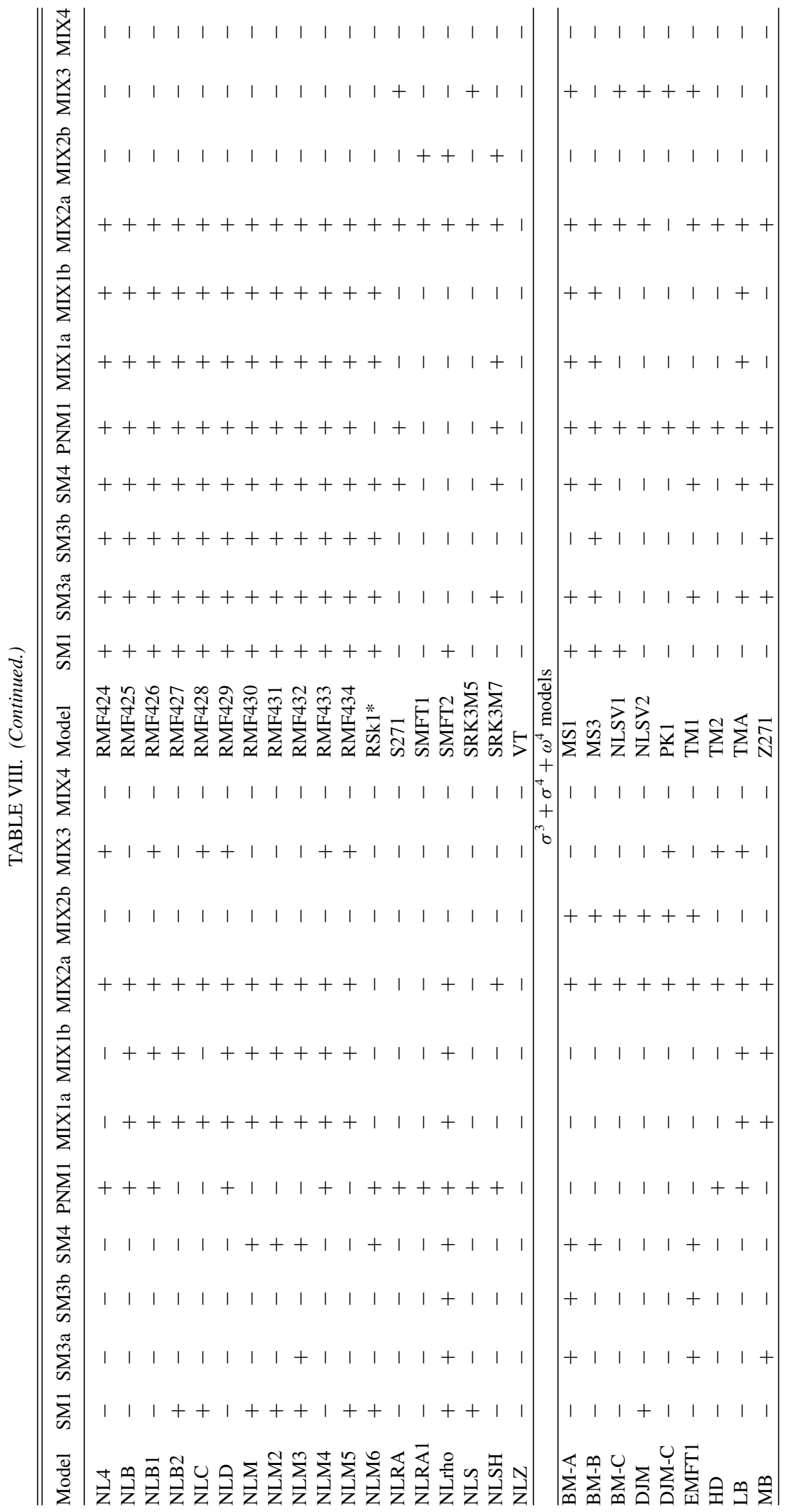




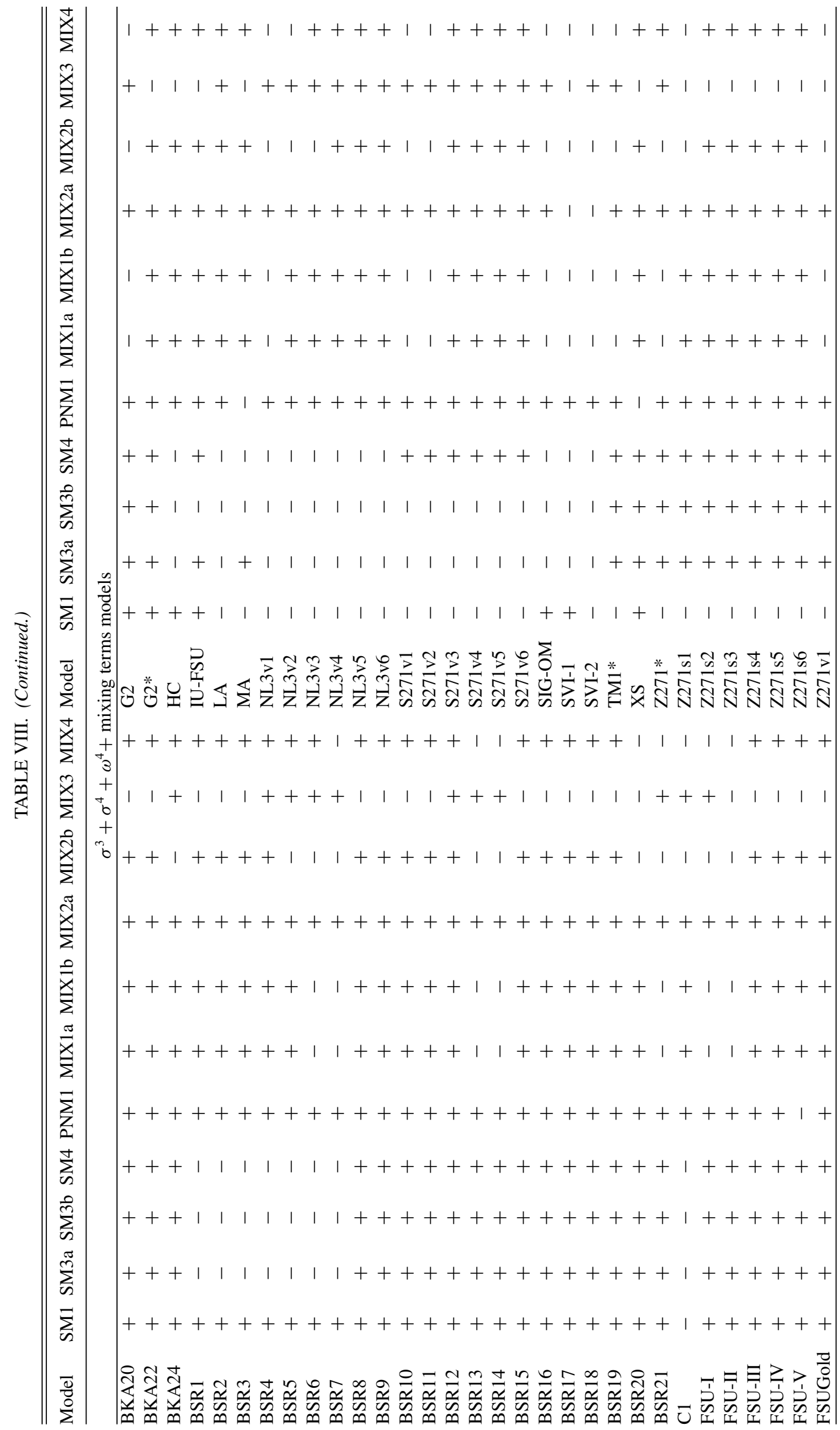




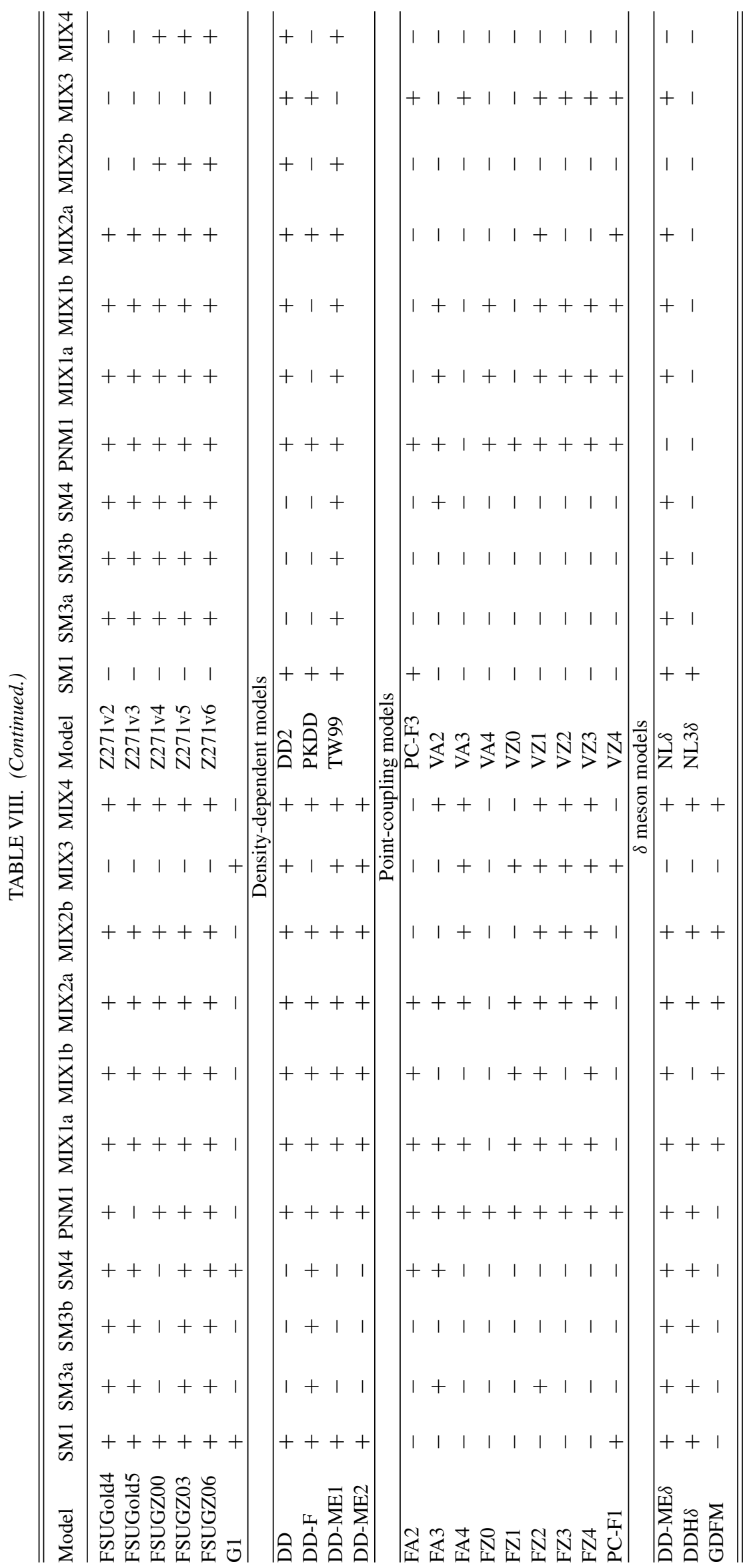


APPENDIX D: DEVIATION TABLE

TABLE IX. List of the RMF parametrizations and their compliance with macroscopic constraints used in this work. For SM1 and MIX1-4 we give the standard deviation; the constraint is satisfied if the standard deviation is less than or equal to one. For SM3, SM4, and PNM1 we give the fraction of the full density range in $\%$ in which the constraint is not satisfied. A letter $\mathrm{L}(\mathrm{H})$ indicates that the RMF prediction starts to fail at the beginning (end) of the density range. No letter indication means that the model fails in the middle of the density range. For models that fail in $100 \%$ of the range, the letter U (D) indicates that its curve is above (below) the band defined by the constraint. For more details, see the text.

\begin{tabular}{|c|c|c|c|c|c|c|c|c|c|c|c|}
\hline Model & SM1 & SM3a $(\%)$ & SM3b $(\%)$ & SM4 (\%) & PNM1 (\%) & MIX1a & MIX1b & $\operatorname{MIX} 2 \mathrm{a}$ & MIX2b & MIX3 & MIX4 \\
\hline \multicolumn{12}{|c|}{ Linear finite-range models } \\
\hline$\overline{\mathrm{H} 1}$ & 7.92 & $100 \mathrm{U}$ & $100 \mathrm{U}$ & $100 \mathrm{U}$ & 0 & -0.81 & -2.63 & 0.41 & 1.34 & -1.56 & -1.62 \\
\hline $\mathrm{L} 2$ & 8.71 & $100 \mathrm{U}$ & $100 \mathrm{U}$ & $100 \mathrm{U}$ & $58.6 \mathrm{H}$ & -2.19 & -5.37 & -0.03 & 0.55 & -0.28 & -1.69 \\
\hline L3 & 9.86 & $100 \mathrm{U}$ & $100 \mathrm{U}$ & $100 \mathrm{U}$ & $75.6 \mathrm{H}$ & -2.23 & -5.46 & -0.01 & 0.58 & -0.34 & -1.77 \\
\hline LBF & 8.85 & $100 \mathrm{U}$ & $100 \mathrm{U}$ & $100 \mathrm{U}$ & 0 & 3.38 & 5.77 & 1.82 & 3.88 & -5.71 & -1.62 \\
\hline LW & 7.89 & $100 \mathrm{U}$ & $100 \mathrm{U}$ & $100 \mathrm{U}$ & $.4 \mathrm{~L}$ & -1.58 & -4.16 & 0.10 & 0.78 & -0.52 & -1.54 \\
\hline $\mathrm{LZ}$ & 8.91 & $100 \mathrm{U}$ & $100 \mathrm{U}$ & $100 \mathrm{U}$ & 0 & 3.77 & 6.54 & 1.95 & 4.12 & -6.14 & -1.62 \\
\hline RMF201 & 8.66 & $100 \mathrm{U}$ & $100 \mathrm{U}$ & $100 \mathrm{U}$ & 0 & 0.50 & 0.00 & 0.85 & 2.13 & -2.82 & -1.62 \\
\hline RMF202 & 8.52 & $100 \mathrm{U}$ & $100 \mathrm{U}$ & $100 \mathrm{U}$ & 0 & 0.50 & 0.00 & 0.85 & 2.13 & -2.83 & -1.62 \\
\hline RMF203 & 8.38 & $100 \mathrm{U}$ & $100 \mathrm{U}$ & $100 \mathrm{U}$ & 0 & 0.50 & 0.00 & 0.85 & 2.13 & -2.84 & -1.62 \\
\hline RMF204 & 8.24 & $100 \mathrm{U}$ & $100 \mathrm{U}$ & $100 \mathrm{U}$ & 0 & 0.50 & 0.00 & 0.85 & 2.13 & -2.85 & -1.62 \\
\hline $\mathrm{E}$ & -0.21 & $100 \mathrm{U}$ & $100 \mathrm{U}$ & $71.4 \mathrm{H}$ & 36.0 & 1.72 & 2.43 & 1.21 & 2.78 & -0.51 & -1.54 \\
\hline ER & -0.24 & $100 \mathrm{U}$ & $100 \mathrm{U}$ & $67.8 \mathrm{H}$ & 13.2 & 1.88 & 2.77 & 1.26 & 2.86 & -0.45 & -1.54 \\
\hline FAMA1 & -0.75 & $100 \mathrm{U}$ & $100 \mathrm{U}$ & $45.6 \mathrm{H}$ & 50.2 & 1.60 & 2.20 & 1.12 & 2.62 & 0.82 & -1.54 \\
\hline FAMA2 & -0.12 & $100 \mathrm{U}$ & $100 \mathrm{U}$ & $58.3 \mathrm{H}$ & 0 & 1.60 & 2.20 & 1.12 & 2.61 & -0.01 & -1.54 \\
\hline FAMA3 & 0.50 & $100 \mathrm{U}$ & $100 \mathrm{U}$ & $73.8 \mathrm{H}$ & 0 & 1.60 & 2.20 & 1.11 & 2.60 & -0.63 & -1.54 \\
\hline FAMA4 & 1.13 & $100 \mathrm{U}$ & $100 \mathrm{U}$ & $89.9 \mathrm{H}$ & 0 & 1.60 & 2.20 & 1.11 & 2.59 & -1.09 & -1.54 \\
\hline FAMA5 & 1.75 & $100 \mathrm{U}$ & $100 \mathrm{U}$ & $100 \mathrm{U}$ & 0 & 1.60 & 2.20 & 1.10 & 2.58 & -1.45 & -1.54 \\
\hline FAMB1 & -0.75 & $8.0 \mathrm{H}$ & $36.4 \mathrm{H}$ & 0 & 0 & 1.40 & 1.80 & 0.85 & 2.14 & 1.70 & -1.38 \\
\hline FAMB2 & -0.12 & $9.3 \mathrm{H}$ & $39.9 \mathrm{H}$ & 0 & 0 & 1.40 & 1.80 & 0.85 & 2.13 & 1.03 & -1.38 \\
\hline FAMB3 & 0.50 & $11.6 \mathrm{H}$ & $17.6 \mathrm{~L} / 51.1 \mathrm{H}$ & 0 & 0 & 1.40 & 1.80 & 0.84 & 2.12 & 0.54 & -1.38 \\
\hline FAMB4 & 1.13 & $8.5 \mathrm{~L} / 14.4 \mathrm{H}$ & $26.2 \mathrm{~L} / 63.7 \mathrm{H}$ & 0 & 0 & 1.40 & 1.80 & 0.84 & 2.11 & 0.19 & -1.38 \\
\hline GL2 & -0.75 & 0 & 0 & 0 & $75.0 \mathrm{H}$ & 0.50 & 0.00 & 0.48 & 1.46 & 1.92 & -1.31 \\
\hline GL3 & -0.75 & 0 & 0 & 0 & $75.8 \mathrm{H}$ & 0.50 & 0.00 & 0.42 & 1.36 & 1.77 & -1.23 \\
\hline GL4 & 0.50 & $17.3 \mathrm{H}$ & 19.6L/57.4H & 0 & 0 & 0.50 & 0.00 & 0.54 & 1.57 & 1.02 & -1.38 \\
\hline GL5 & 0.50 & 0 & $2.3 \mathrm{~L}$ & 0 & 0 & 0.50 & 0.00 & 0.47 & 1.45 & 1.14 & -1.31 \\
\hline GL6 & 0.50 & 0 & 0 & 0 & 0 & 0.50 & 0.00 & 0.42 & 1.35 & 1.33 & -1.23 \\
\hline GL7 & 1.75 & $3.5 \mathrm{~L}$ & $17.2 \mathrm{~L}$ & $3.7 \mathrm{~L} / 1.0$ & 0 & 0.50 & 0.00 & 0.46 & 1.44 & 0.79 & -1.31 \\
\hline GL8 & 1.75 & 0 & $7.5 \mathrm{~L}$ & $1.0 \mathrm{~L}$ & 0 & 0.50 & 0.00 & 0.41 & 1.34 & 1.22 & -1.23 \\
\hline GL82 & 1.39 & 0 & $6.5 \mathrm{~L}$ & 0 & 0 & 1.25 & 1.49 & 0.70 & 1.85 & 0.63 & -1.31 \\
\hline GL9 & -0.50 & 0 & 0 & 0 & $70.8 \mathrm{H}$ & 0.50 & 0.00 & 0.44 & 1.40 & 1.66 & -1.31 \\
\hline GM1 & 1.76 & $17.3 \mathrm{~L} / 23.3 \mathrm{H}$ & $100 \mathrm{U}$ & $100 \mathrm{U}$ & 0 & 0.50 & 0.01 & 0.53 & 1.56 & 0.48 & -1.38 \\
\hline GM2 & 1.75 & 0 & $11.5 \mathrm{~L}$ & $1.6 \mathrm{~L} / .8$ & 0 & 0.50 & 0.00 & 0.43 & 1.37 & 1.02 & -1.23 \\
\hline GM3 & 0.25 & 0 & 0 & 0 & 0 & 0.50 & 0.00 & 0.44 & 1.39 & 1.31 & -1.23 \\
\hline GPS & 1.74 & 0 & $6.3 \mathrm{~L}$ & 0 & 0 & 0.50 & 0.00 & 0.41 & 1.34 & 1.18 & -1.23 \\
\hline
\end{tabular}


TABLE IX. (Continued.)

\begin{tabular}{|c|c|c|c|c|c|c|c|c|c|c|c|}
\hline Hybrid & 0.00 & $100 \mathrm{U}$ & $100 \mathrm{U}$ & $63.7 \mathrm{H}$ & 42.2 & 1.46 & 1.92 & 1.08 & 2.54 & -0.09 & -1.54 \\
\hline MS2 & 0.50 & $100 \mathrm{U}$ & $100 \mathrm{U}$ & $74.3 \mathrm{H}$ & 0 & 1.00 & 1.00 & 0.91 & 2.24 & -0.33 & -1.54 \\
\hline NL06 & -0.87 & $100 \mathrm{U}$ & $100 \mathrm{U}$ & $40.3 \mathrm{H}$ & 37.8 & 1.87 & 2.73 & 1.20 & 2.77 & 1.00 & -1.54 \\
\hline NL065 & 0.67 & $100 \mathrm{U}$ & $100 \mathrm{U}$ & $37.2 \mathrm{H}$ & 0 & 1.80 & 2.59 & 1.06 & 2.51 & 0.00 & -1.46 \\
\hline NL07 & 1.16 & $8.9 \mathrm{~L} / 15.8 \mathrm{H}$ & $26.5 \mathrm{~L} / 65.2 \mathrm{H}$ & 0 & 0 & 1.70 & 2.41 & 0.94 & 2.29 & 0.12 & -1.38 \\
\hline NL1 & -0.47 & $100 \mathrm{U}$ & $100 \mathrm{U}$ & $70.8 \mathrm{H}$ & 0 & 2.69 & 4.38 & 1.56 & 3.40 & -0.84 & -1.54 \\
\hline NL1J4 & -0.46 & $100 U$ & $100 \mathrm{U}$ & $72.7 \mathrm{H}$ & 47.2 & 2.00 & 3.00 & 1.33 & 3.00 & -0.58 & -1.54 \\
\hline NL1J5 & -0.46 & $100 \mathrm{U}$ & $100 \mathrm{U}$ & $72.7 \mathrm{H}$ & 0 & 4.00 & 7.00 & 2.00 & 4.20 & -1.78 & -1.54 \\
\hline NL2 & 4.23 & $100 U$ & $100 \mathrm{U}$ & $100 \mathrm{U}$ & 0 & 2.77 & 4.54 & 1.33 & 2.99 & -1.53 & -1.46 \\
\hline NL3 & 1.04 & $100 \mathrm{U}$ & $100 \mathrm{U}$ & $88.5 \mathrm{H}$ & 0 & 1.48 & 1.96 & 1.08 & 2.54 & -0.99 & -1.54 \\
\hline NL3-II & 1.04 & $100 \mathrm{U}$ & $100 \mathrm{U}$ & $90.1 \mathrm{H}$ & 0 & 1.54 & 2.08 & 1.10 & 2.59 & -1.09 & -1.54 \\
\hline NLB2 & 0.39 & $100 \mathrm{U}$ & $100 \mathrm{U}$ & $100 U$ & $68.0 \mathrm{H}$ & 0.62 & 0.24 & 0.92 & 2.25 & -1.37 & -1.62 \\
\hline NLC & -0.14 & $100 \mathrm{U}$ & $100 \mathrm{U}$ & $37.2 \mathrm{H}$ & 25.8 & 1.00 & 1.01 & 0.84 & 2.12 & 0.75 & -1.46 \\
\hline NLD & 2.83 & $23.1 \mathrm{~L} / 27.2 \mathrm{H}$ & $100 \mathrm{U}$ & $100 \mathrm{U}$ & 0 & 1.00 & 1.00 & 0.70 & 1.86 & -0.16 & -1.38 \\
\hline NLM & -0.75 & $21.0 \mathrm{H}$ & $45.5 \mathrm{H}$ & 0 & $74.0 \mathrm{H}$ & 0.00 & -1.00 & 0.38 & 1.28 & 2.15 & -1.31 \\
\hline NLM2 & -0.75 & $19.4 \mathrm{H}$ & $41.6 \mathrm{H}$ & 0 & $81.4 \mathrm{H}$ & 0.00 & -1.00 & 0.38 & 1.28 & 2.37 & -1.31 \\
\hline NLM3 & -0.75 & $2.7 \mathrm{H}$ & $33.7 \mathrm{H}$ & 0 & $81.0 \mathrm{H}$ & 0.00 & -1.00 & 0.39 & 1.30 & 2.11 & -1.38 \\
\hline NLM4 & 1.75 & $21.4 \mathrm{~L} / 35.0 \mathrm{H}$ & $100 U$ & $100 \mathrm{U}$ & 0 & 0.00 & -1.00 & 0.36 & 1.25 & 0.71 & -1.31 \\
\hline NLM5 & -0.75 & $100 \mathrm{U}$ & $100 \mathrm{U}$ & $82.5 \mathrm{H}$ & $83.4 \mathrm{H}$ & 0.00 & -1.00 & 0.74 & 1.93 & -0.01 & -1.62 \\
\hline NLM6 & -0.75 & $21.0 \mathrm{H}$ & $45.5 \mathrm{H}$ & 0 & 0 & 2.00 & 3.00 & 1.04 & 2.48 & 1.55 & -1.38 \\
\hline NLRA & 2.26 & $100 \mathrm{U}$ & $100 \mathrm{U}$ & $100 \mathrm{U}$ & 0 & 1.78 & 2.56 & 1.09 & 2.56 & -1.22 & -1.46 \\
\hline NLRA1 & 1.38 & $100 \mathrm{U}$ & $100 \mathrm{U}$ & $1.7 \mathrm{~L} / 98.3 \mathrm{H}$ & 0 & 1.29 & 1.58 & 1.01 & 2.42 & -1.06 & -1.54 \\
\hline P-075 & 1.03 & $5.3 \mathrm{~L}$ & 18.7L/16.7H & $19.0 \mathrm{~L} / 5.0$ & 0 & 2.59 & 4.18 & 1.14 & 2.66 & 0.11 & -1.31 \\
\hline P-080 & 0.75 & 0 & $3.0 \mathrm{~L}$ & 0 & 0 & 1.93 & 2.85 & 0.88 & 2.19 & 0.67 & -1.31 \\
\hline Q1 & 0.30 & $100 \mathrm{U}$ & $100 \mathrm{U}$ & $69.5 \mathrm{H}$ & 26.6 & 1.29 & 1.58 & 1.02 & 2.43 & -0.29 & -1.54 \\
\hline RMF301 & 0.60 & 0 & 0 & 0 & 0 & 0.50 & 0.00 & 0.44 & 1.39 & 1.18 & -1.31 \\
\hline RMF302 & 0.49 & 0 & 0 & 0 & 0 & 0.50 & 0.00 & 0.44 & 1.39 & 1.23 & -1.23 \\
\hline RMF303 & 0.47 & 0 & 0 & 0 & 0 & 0.50 & 0.00 & 0.44 & 1.38 & 1.24 & -1.23 \\
\hline RMF304 & 0.45 & 0 & 0 & 0 & 0 & 0.50 & 0.00 & 0.43 & 1.38 & 1.25 & -1.23 \\
\hline RMF305 & 0.41 & 0 & 0 & 0 & 0 & 0.50 & 0.00 & 0.43 & 1.38 & 1.27 & -1.23 \\
\hline RMF306 & 0.37 & 0 & 0 & 0 & 0 & 0.50 & 0.00 & 0.43 & 1.38 & 1.29 & -1.23 \\
\hline RMF307 & 0.35 & 0 & 0 & 0 & 0 & 0.50 & 0.00 & 0.43 & 1.37 & 1.30 & -1.23 \\
\hline RMF308 & 0.32 & 0 & 0 & 0 & 0 & 0.50 & 0.00 & 0.43 & 1.37 & 1.31 & -1.23 \\
\hline RMF309 & 0.28 & 0 & 0 & 0 & 0 & 0.50 & 0.00 & 0.43 & 1.37 & 1.33 & -1.23 \\
\hline RMF310 & 0.22 & 0 & 0 & 0 & 0 & 0.50 & 0.00 & 0.42 & 1.36 & 1.36 & -1.23 \\
\hline RMF311 & 0.20 & 0 & 0 & 0 & 0 & 0.50 & 0.00 & 0.42 & 1.36 & 1.37 & -1.23 \\
\hline RMF312 & 0.18 & 0 & 0 & 0 & 0 & 0.50 & 0.00 & 0.42 & 1.36 & 1.38 & -1.23 \\
\hline RMF313 & 0.13 & 0 & 0 & 0 & 0 & 0.50 & 0.00 & 0.42 & 1.36 & 1.40 & -1.23 \\
\hline RMF314 & 0.11 & 0 & 0 & 0 & 0 & 0.50 & 0.00 & 0.42 & 1.36 & 1.41 & -1.23 \\
\hline RMF315 & 0.10 & 0 & 0 & 0 & 0 & 0.50 & 0.00 & 0.42 & 1.36 & 1.41 & -1.23 \\
\hline RMF316 & 0.09 & 0 & 0 & 0 & 0 & 0.50 & 0.00 & 0.42 & 1.36 & 1.42 & -1.23 \\
\hline RMF317 & 0.07 & 0 & 0 & 0 & 0 & 0.50 & 0.00 & 0.42 & 1.35 & 1.43 & -1.23 \\
\hline RMF401 & 0.00 & $.4 \mathrm{H}$ & $34.2 \mathrm{H}$ & 0 & 52.8 & 0.50 & 0.00 & 0.53 & 1.55 & 1.37 & -1.31 \\
\hline RMF402 & 0.05 & $.5 \mathrm{H}$ & $34.6 \mathrm{H}$ & 0 & 49.2 & 0.50 & 0.00 & 0.53 & 1.55 & 1.33 & -1.31 \\
\hline
\end{tabular}


TABLE IX. (Continued.)

\begin{tabular}{|c|c|c|c|c|c|c|c|c|c|c|c|}
\hline Model & SM1 & SM3a $(\%)$ & SM3b (\%) & SM4 (\%) & PNM1 (\%) & MIX1a & MIX1b & MIX2a & MIX2b & MIX3 & MIX4 \\
\hline RMF403 & 0.00 & 0 & $21.7 \mathrm{H}$ & 0 & 49.8 & 0.50 & 0.00 & 0.51 & 1.53 & 1.38 & -1.31 \\
\hline RMF404 & 0.05 & 0 & $22.1 \mathrm{H}$ & 0 & 45.6 & 0.50 & 0.00 & 0.51 & 1.52 & 1.34 & -1.31 \\
\hline RMF406 & 0.10 & 0 & 0 & 0 & 0 & 0.50 & 0.00 & 0.44 & 1.39 & 1.37 & -1.31 \\
\hline RMF407 & 0.00 & 0 & $8.2 \mathrm{H}$ & 0 & 46.6 & 0.50 & 0.00 & 0.50 & 1.50 & 1.38 & -1.31 \\
\hline RMF408 & 0.05 & 0 & $8.7 \mathrm{H}$ & 0 & 42.0 & 0.50 & 0.00 & 0.50 & 1.50 & 1.35 & -1.31 \\
\hline RMF410 & 0.15 & 0 & $9.8 \mathrm{H}$ & 0 & 31.2 & 0.50 & 0.00 & 0.50 & 1.50 & 1.29 & -1.31 \\
\hline RMF411 & 0.00 & 0 & 0 & 0 & 43.6 & 0.50 & 0.00 & 0.49 & 1.48 & 1.38 & -1.31 \\
\hline RMF412 & 0.05 & 0 & 0 & 0 & 38.2 & 0.50 & 0.00 & 0.49 & 1.48 & 1.35 & -1.31 \\
\hline RMF413 & 0.10 & 0 & 0 & 0 & 32.4 & 0.50 & 0.00 & 0.49 & 1.47 & 1.32 & -1.31 \\
\hline RMF414 & 0.15 & 0 & 0 & 0 & 25.0 & 0.50 & 0.00 & 0.49 & 1.47 & 1.30 & -1.31 \\
\hline RMF415 & 0.00 & 0 & 0 & 0 & 40.0 & 0.50 & 0.00 & 0.47 & 1.45 & 1.39 & -1.31 \\
\hline RMF420 & 0.05 & 0 & 0 & 0 & 29.2 & 0.50 & 0.00 & 0.46 & 1.43 & 1.37 & -1.31 \\
\hline RMF421 & 0.10 & 0 & 0 & 0 & 19.6 & 0.50 & 0.00 & 0.46 & 1.43 & 1.34 & -1.31 \\
\hline RMF422 & 0.00 & 0 & 0 & 0 & 31.4 & 0.50 & 0.00 & 0.45 & 1.41 & 1.40 & -1.31 \\
\hline RMF423 & 0.05 & 0 & 0 & 0 & 22.2 & 0.50 & 0.00 & 0.45 & 1.41 & 1.37 & -1.31 \\
\hline RMF424 & 0.40 & 0 & 0 & 0 & 0 & 0.50 & 0.00 & 0.43 & 1.37 & 1.30 & -1.23 \\
\hline RMF425 & 0.45 & 0 & 0 & 0 & 0 & 0.50 & 0.00 & 0.43 & 1.37 & 1.29 & -1.23 \\
\hline RMF426 & 0.50 & 0 & 0 & 0 & 0 & 0.50 & 0.00 & 0.43 & 1.37 & 1.27 & -1.23 \\
\hline RMF427 & 0.15 & 0 & 0 & 0 & 0 & 0.50 & 0.00 & 0.42 & 1.35 & 1.41 & -1.23 \\
\hline RMF428 & 0.20 & 0 & 0 & 0 & 0 & 0.50 & 0.00 & 0.42 & 1.35 & 1.39 & -1.23 \\
\hline RMF429 & 0.25 & 0 & 0 & 0 & 0 & 0.50 & 0.00 & 0.42 & 1.35 & 1.38 & -1.23 \\
\hline RMF430 & 0.30 & 0 & 0 & 0 & 0 & 0.50 & 0.00 & 0.42 & 1.35 & 1.37 & -1.23 \\
\hline SRK3M5 & 1.75 & $100 \mathrm{U}$ & $100 \mathrm{U}$ & $100 \mathrm{U}$ & $84.6 \mathrm{H}$ & -1.30 & -3.60 & 0.28 & 1.10 & -0.42 & -1.62 \\
\hline SRK3M7 & 1.75 & $1.5 \mathrm{~L}$ & $16.3 \mathrm{~L}$ & $1.2 \mathrm{~L}$ & 0 & -0.25 & -1.51 & 0.22 & 0.99 & 1.11 & -1.31 \\
\hline VT & -1.43 & $100 \mathrm{U}$ & $100 \mathrm{U}$ & $43.9 \mathrm{H}$ & 56.6 & 1.94 & 2.89 & 1.26 & 2.87 & 1.82 & -1.54 \\
\hline \multicolumn{12}{|c|}{$\sigma^{3}+\sigma^{4}+\omega^{4}$ models } \\
\hline$\overline{\mathrm{BM}-\mathrm{A}}$ & -1.04 & 0 & 0 & 0 & $88.8 \mathrm{H}$ & -2.08 & -5.15 & -0.40 & -0.12 & 2.27 & -1.15 \\
\hline BM-B & -1.48 & 8.2 & 8.2 & 0 & $84.6 \mathrm{H}$ & -2.52 & -6.03 & -0.55 & -0.38 & 2.64 & -1.15 \\
\hline BM-C & -1.67 & $90.7 \mathrm{~L}$ & $90.7 \mathrm{~L}$ & $5.8 \mathrm{H} / 1.8$ & $80.4 \mathrm{H}$ & -2.80 & -6.60 & -0.63 & -0.54 & 2.84 & -1.15 \\
\hline DJM & 0.37 & $16.6 \mathrm{~L}$ & $28.2 \mathrm{~L}$ & $.1 \mathrm{~L} / 87.2 \mathrm{H}$ & $80.2 \mathrm{H}$ & -1.96 & -4.92 & -0.16 & 0.32 & 1.11 & -1.46 \\
\hline DJM-C & 2.49 & $26.9 \mathrm{~L}$ & $100 \mathrm{U}$ & $100 \mathrm{U}$ & $66.2 \mathrm{H}$ & -1.62 & -4.24 & -0.03 & 0.54 & 0.72 & -1.54 \\
\hline EMFT1 & -1.60 & 0 & 0 & 0 & $88.8 \mathrm{H}$ & -2.28 & -5.56 & -0.30 & 0.06 & 1.60 & -1.38 \\
\hline HD & 1.35 & $91.1 \mathrm{~L}$ & $100 \mathrm{U}$ & $100 \mathrm{U}$ & 0 & 1.14 & 1.28 & 0.80 & 2.04 & -0.02 & -1.38 \\
\hline LB & 2.17 & $26.2 \mathrm{~L}$ & $100 \mathrm{U}$ & $100 \mathrm{U}$ & 0 & 0.38 & -0.23 & 0.59 & 1.67 & 0.08 & -1.46 \\
\hline MB & 2.79 & $1.7 \mathrm{~L}$ & $12.1 \mathrm{~L}$ & $55.7 \mathrm{~L} / 9.1$ & $100 \mathrm{D}$ & 0.50 & 0.00 & 0.44 & 1.40 & 2.30 & -1.31 \\
\hline MS1 & 0.50 & 0 & $16.8 \mathrm{~L}$ & 0 & 0 & 1.00 & 1.00 & 0.82 & 2.07 & 0.11 & -1.46 \\
\hline MS3 & 0.50 & 0 & 0 & 0 & 0 & 1.00 & 1.00 & 0.72 & 1.90 & 1.39 & -1.46 \\
\hline NLSV1 & 1.00 & $24.0 \mathrm{~L}$ & $100 \mathrm{U}$ & $58.8 \mathrm{H}$ & 0 & 1.46 & 1.92 & 0.99 & 2.39 & 1.11 & -1.46 \\
\hline NLSV2 & 1.54 & $11.6 \mathrm{~L}$ & $24.2 \mathrm{~L}$ & $37.2 \mathrm{H}$ & 0 & 1.35 & 1.70 & 0.92 & 2.26 & -0.32 & -1.46 \\
\hline PK1 & 1.32 & $21.5 \mathrm{~L}$ & $100 \mathrm{U}$ & $76.0 \mathrm{H}$ & 0 & 1.53 & 2.06 & 1.02 & 2.44 & -0.08 & -1.46 \\
\hline TM1 & 1.28 & $1.1 \mathrm{~L}$ & $17.3 \mathrm{~L}$ & 0 & 0 & 1.38 & 1.76 & 0.91 & 2.23 & -0.52 & -1.46 \\
\hline TM2 & 2.85 & $20.7 \mathrm{~L}$ & $49.6 \mathrm{~L}$ & $100 \mathrm{U}$ & 0 & 1.20 & 1.39 & 0.96 & 2.32 & 0.21 & -1.54 \\
\hline TMA & 2.20 & 0 & $9.3 \mathrm{~L}$ & $3.8 \mathrm{~L}$ & 0 & 0.13 & -0.74 & 0.45 & 1.41 & -1.06 & -1.38 \\
\hline Z271 & 1.02 & 0 & 0 & 0 & 0 & 1.18 & 1.37 & 0.64 & 1.75 & 1.21 & -1.31 \\
\hline
\end{tabular}


TABLE IX. (Continued.)

\begin{tabular}{|c|c|c|c|c|c|c|c|c|c|c|c|}
\hline Model & SM1 & SM3a $(\%)$ & $\operatorname{SM} 3 \mathrm{~b}(\%)$ & SM4 (\%) & PNM1 $(\%)$ & MIX1a & MIX1b & $\operatorname{MIX} 2 \mathrm{a}$ & MIX2b & MIX3 & MIX4 \\
\hline \multicolumn{12}{|c|}{$\sigma^{3}+\sigma^{4}+\omega^{4}+$ mixing-terms models } \\
\hline$\overline{\text { BKA20 }}$ & 0.20 & 0 & 0 & 0 & 0 & 0.45 & -0.10 & 0.12 & 0.82 & 1.53 & -0.85 \\
\hline BKA24 & -0.07 & 0 & 0 & 0 & 0 & 0.84 & 0.68 & 0.33 & 1.19 & 0.86 & -1.00 \\
\hline BSR1 & 0.25 & 1.00 & $100 \mathrm{U}$ & $37.2 \mathrm{H}$ & 0 & 100 & -0.58 & 0.18 & 1.44 & 1.44 & 1.00 \\
\hline BSR2 & 0.25 & $16.8 \mathrm{~L} / 27.9 \mathrm{H}$ & $100 \mathrm{U}$ & $37.2 \mathrm{H}$ & 0 & 0.30 & -0.40 & -0.18 & 0.28 & 1.25 & -0.38 \\
\hline BSR4 & 0.21 & $27.8 \mathrm{~L} / 69.0 \mathrm{H}$ & $100 \mathrm{U}$ & $37.2 \mathrm{H}$ & 0 & 0.63 & 0.27 & 0.07 & 0.73 & 0.59 & -0.69 \\
\hline BSR5 & 0.14 & $100 \mathrm{U}$ & $100 \mathrm{U}$ & $38.5 \mathrm{H}$ & 0 & 0.89 & 0.78 & 0.30 & 1.13 & 0.26 & -0.92 \\
\hline BSR6 & 0.14 & $26.0 \mathrm{~L} / 63.4 \mathrm{H}$ & $100 \mathrm{U}$ & $37.2 \mathrm{H}$ & 0 & 1.12 & 1.25 & 0.35 & 1.23 & -0.07 & -1.00 \\
\hline BSR7 & 0.05 & $100 \mathrm{U}$ & $100 \mathrm{U}$ & $38.3 \mathrm{H}$ & 0 & 1.45 & 1.90 & 0.65 & 1.77 & -0.36 & -1.23 \\
\hline BSR8 & 0.02 & 0 & $1.5 \mathrm{~L}$ & 0 & 0 & 0.22 & -0.57 & -0.22 & 0.21 & 1.76 & -0.31 \\
\hline BSR9 & 0.06 & 0 & 0 & 0 & 0 & 0.32 & -0.36 & -0.14 & 0.36 & 1.58 & -0.38 \\
\hline BSR14 & 0.14 & 0 & 0 & 0 & 0 & 1.26 & 1.53 & 0.53 & 1.55 & 0.48 & -1.15 \\
\hline BSR15 & -0.08 & 0 & 0 & 0 & 0 & 0.19 & -0.61 & -0.18 & 0.27 & 1.98 & -0.38 \\
\hline BSR16 & -0.13 & 0 & 0 & 0 & 0 & 0.25 & -0.50 & -0.17 & 0.29 & 1.94 & -0.38 \\
\hline BSR17 & -0.21 & 0 & 0 & 0 & 0 & 0.40 & -0.21 & -0.06 & 0.50 & 1.75 & -0.54 \\
\hline BSR18 & -0.22 & 0 & 0 & 0 & 0 & 0.55 & 0.10 & 0.06 & 0.71 & 1.54 & -0.77 \\
\hline BSR19 & -0.23 & 0 & 0 & 0 & 0 & 0.76 & 0.51 & 0.21 & 0.98 & 1.32 & -0.92 \\
\hline BSR20 & -0.17 & 0 & 0 & 0 & 0 & 0.91 & 0.82 & 0.40 & 1.32 & 1.21 & -1.08 \\
\hline BSR21 & -0.24 & 0 & 0 & 0 & 0 & 1.19 & 1.38 & 0.51 & 1.52 & 0.96 & -1.15 \\
\hline $\mathrm{C} 1$ & 1.85 & $17.6 \mathrm{~L} / 20.6 \mathrm{H}$ & $100 \mathrm{U}$ & $3.0 \mathrm{~L} / 88.1 \mathrm{H}$ & 0 & 0.41 & -0.19 & 0.55 & 1.58 & 0.33 & -1.38 \\
\hline FSU-I & -0.01 & 0 & 0 & 0 & 0 & 1.48 & 1.97 & 0.88 & 2.18 & 0.97 & -1.46 \\
\hline FSU-II & -0.01 & 0 & 0 & 0 & 0 & 1.10 & 1.20 & 0.39 & 1.30 & 1.05 & -1.08 \\
\hline FSUGZ03 & 0.06 & 0 & 0 & 0 & 0 & 0.31 & -0.38 & -0.13 & 0.36 & 1.57 & -0.46 \\
\hline FSUGZ06 & -0.12 & 0 & 0 & 0 & 0 & 0.24 & -0.53 & -0.17 & 0.30 & 1.94 & -0.38 \\
\hline G1 & -0.38 & 6.5 & $25.9 \mathrm{~L}$ & 0 & 33.0 & 1.70 & 2.39 & 1.18 & 2.73 & 0.77 & -1.54 \\
\hline $\mathrm{G} 2$ & -0.38 & 0 & 0 & 0 & 0 & 1.28 & 1.56 & 0.68 & 1.83 & 0.96 & -1.31 \\
\hline $\mathrm{G} 2 *$ & -0.38 & 0 & 0 & 0 & 0 & 0.08 & -0.84 & -0.01 & 0.59 & 1.68 & -0.77 \\
\hline $\mathrm{HC}$ & 0.05 & $18.8 \mathrm{~L} / 64.6 \mathrm{H}$ & $100 \mathrm{U}$ & $6.4 \mathrm{H}$ & 0 & 0.20 & -0.60 & -0.25 & 0.14 & 1.32 & -0.62 \\
\hline IU-FSU & 0.03 & 0 & $11.5 \mathrm{~L}$ & 0 & 0 & 0.26 & -0.48 & -0.51 & -0.31 & 2.36 & 0.23 \\
\hline LA & 1.79 & $20.6 \mathrm{~L}$ & $50.5 \mathrm{~L}$ & $100 \mathrm{U}$ & 0 & 0.70 & 0.39 & 0.19 & 0.95 & 0.36 & -1.00 \\
\hline MA & 2.93 & $.2 \mathrm{~L}$ & $11.2 \mathrm{~L}$ & $50.2 \mathrm{~L} / 5.5$ & $100 \mathrm{D}$ & 0.48 & -0.04 & 0.03 & 0.66 & 2.07 & -0.85 \\
\hline NL3v1 & 1.04 & $100 \mathrm{U}$ & $100 \mathrm{U}$ & $88.7 \mathrm{H}$ & 0 & 1.21 & 1.43 & 0.69 & 1.85 & -0.89 & -1.31 \\
\hline NL3v2 & 1.04 & $100 \mathrm{U}$ & $100 \mathrm{U}$ & $88.7 \mathrm{H}$ & 0 & 1.00 & 0.99 & 0.39 & 1.31 & -0.59 & -1.15 \\
\hline NL3v3 & 1.04 & $100 \mathrm{U}$ & $100 \mathrm{U}$ & $88.7 \mathrm{H}$ & 0 & 0.90 & 0.79 & 0.27 & 1.08 & -0.40 & -1.00 \\
\hline NL3v4 & 1.04 & $100 \mathrm{U}$ & $100 \mathrm{U}$ & $88.7 \mathrm{H}$ & 0 & 0.80 & 0.61 & 0.15 & 0.88 & -0.20 & -0.92 \\
\hline NL3v5 & 1.04 & $100 \mathrm{U}$ & $100 \mathrm{U}$ & $88.7 \mathrm{H}$ & 0 & 0.63 & 0.26 & -0.04 & 0.53 & 0.24 & -0.69 \\
\hline NL3v6 & 1.04 & $100 \mathrm{U}$ & $100 \mathrm{U}$ & $88.7 \mathrm{H}$ & 0 & 0.48 & -0.04 & -0.20 & 0.25 & 0.69 & -0.54 \\
\hline S271v1 & 1.02 & $5.5 \mathrm{~L} / 13.2 \mathrm{H}$ & $24.6 \mathrm{~L} / 60.0 \mathrm{H}$ & 0 & 0 & 1.15 & 1.29 & 0.58 & 1.64 & 0.23 & -1.23 \\
\hline S271v2 & 1.02 & $5.5 \mathrm{~L} / 13.2 \mathrm{H}$ & $24.6 \mathrm{~L} / 60.0 \mathrm{H}$ & 0 & 0 & 1.01 & 1.02 & 0.37 & 1.27 & 0.22 & -1.15 \\
\hline S271v3 & 1.02 & $5.5 \mathrm{~L} / 13.2 \mathrm{H}$ & $24.6 \mathrm{~L} / 60.0 \mathrm{H}$ & 0 & 0 & 0.88 & 0.77 & 0.20 & 0.95 & 0.28 & -1.00 \\
\hline S271v4 & 1.02 & $5.5 \mathrm{~L} / 13.2 \mathrm{H}$ & $24.6 \mathrm{~L} / 60.0 \mathrm{H}$ & 0 & 0 & 0.77 & 0.53 & 0.04 & 0.67 & 0.39 & -0.92 \\
\hline S271v5 & 1.02 & $5.5 \mathrm{~L} / 13.2 \mathrm{H}$ & $24.6 \mathrm{~L} / 60.0 \mathrm{H}$ & 0 & 0 & 0.65 & 0.31 & -0.10 & 0.42 & 0.53 & -0.77 \\
\hline S271v6 & 1.02 & $5.5 \mathrm{~L} / 13.2 \mathrm{H}$ & $24.6 \mathrm{~L} / 60.0 \mathrm{H}$ & 0 & 0 & 0.55 & 0.10 & -0.23 & 0.19 & 0.71 & -0.69 \\
\hline SIG-OM & 0.88 & $12.2 \mathrm{~L} / 0.3 \mathrm{H}$ & $28.4 \mathrm{~L} / 69.9 \mathrm{H}$ & $5.0 \mathrm{H} / 6.8$ & 0 & 1.40 & 1.80 & 0.93 & 2.28 & 0.12 & -1.46 \\
\hline
\end{tabular}


TABLE IX. (Continued.)

\begin{tabular}{|c|c|c|c|c|c|c|c|c|c|c|c|}
\hline SVI-1 & 0.85 & $100 \mathrm{U}$ & $100 \mathrm{U}$ & $44.0 \mathrm{H}$ & 0 & 1.41 & 1.82 & 1.03 & 2.46 & 1.09 & -1.46 \\
\hline SVI-2 & 1.04 & $100 \mathrm{U}$ & $100 \mathrm{U}$ & $45.0 \mathrm{H}$ & 0 & 1.41 & 1.82 & 1.02 & 2.44 & 0.93 & -1.46 \\
\hline XS & 0.00 & 0 & 0 & 0 & $73.8 \mathrm{H}$ & 0.37 & -0.27 & -0.33 & 0.00 & 2.40 & -0.08 \\
\hline $\mathrm{Z} 271^{*}$ & 1.02 & 0 & 0 & 0 & 0 & 2.05 & 3.10 & 0.30 & 1.14 & 0.51 & -0.92 \\
\hline Z271s1 & 1.02 & 0 & 0 & 0 & 0 & 0.99 & 0.98 & 0.37 & 1.27 & 1.33 & -1.08 \\
\hline Z271s3 & 1.02 & 0 & 0 & 0 & 0 & 0.65 & 0.31 & -0.05 & 0.51 & 1.48 & -0.77 \\
\hline Z271s4 & 1.02 & 0 & 0 & 0 & 0 & 0.51 & 0.01 & -0.22 & 0.21 & 1.64 & -0.54 \\
\hline Z271s5 & 1.02 & 0 & 0 & 0 & 0 & 0.37 & -0.26 & -0.37 & -0.06 & 1.83 & -0.38 \\
\hline Z271s6 & 1.02 & 0 & 0 & 0 & 0 & 0.24 & -0.52 & -0.49 & -0.29 & 2.03 & -0.15 \\
\hline Z271v1 & 1.02 & 0 & 0 & 0 & 0 & 1.07 & 1.14 & 0.46 & 1.43 & 1.23 & -1.15 \\
\hline Z271v2 & 1.02 & 0 & 0 & 0 & 0 & 0.96 & 0.92 & 0.30 & 1.14 & 1.13 & -1.08 \\
\hline \multicolumn{12}{|c|}{ Density-dependent models } \\
\hline$\overline{\mathrm{DD}}$ & 0.25 & $22.0 \mathrm{~L} / 37.7 \mathrm{H}$ & $100 \mathrm{U}$ & $37.2 \mathrm{H}$ & 0 & 0.33 & -0.34 & -0.31 & 0.04 & 0.59 & -0.46 \\
\hline DD-F & -0.17 & 0 & 0 & 0 & 0 & 0.33 & -0.35 & -0.31 & 0.04 & -0.21 & -0.54 \\
\hline DD-ME1 & 0.37 & $100 \mathrm{U}$ & $100 \mathrm{U}$ & $69.0 \mathrm{H}$ & 0 & 0.61 & 0.22 & -0.32 & 0.02 & 1.76 & -0.31 \\
\hline DD-ME2 & 0.52 & $100 \mathrm{U}$ & $100 \mathrm{U}$ & $82.3 \mathrm{H}$ & 0 & 0.46 & -0.08 & -0.42 & -0.15 & 0.30 & -0.23 \\
\hline DD2 & 0.32 & $24.3 \mathrm{~L} / 40.7 \mathrm{H}$ & $100 \mathrm{U}$ & $41.5 \mathrm{H}$ & 0 & 0.33 & -0.33 & -0.33 & 0.00 & 1.45 & -0.38 \\
\hline PKDD & 0.80 & $15.7 \mathrm{~L} / 18.0 \mathrm{H}$ & $100 \mathrm{U}$ & $37.2 \mathrm{H}$ & 0 & 1.36 & 1.72 & 0.45 & 1.41 & 0.38 & -1.08 \\
\hline TW99 & 0.26 & 0 & 0 & 0 & 0 & 0.55 & 0.11 & -0.33 & 0.01 & 0.58 & -0.38 \\
\hline \multicolumn{12}{|c|}{ Point-coupling models } \\
\hline FA2 & 1.43 & 91.9L & 91.9L & 0 & 0 & 0.71 & 0.41 & 0.65 & 1.78 & 0.70 & -1.46 \\
\hline FA3 & 1.14 & 0 & $15.5 \mathrm{~L}$ & 0 & 0 & -0.06 & -1.12 & -0.91 & -1.04 & 0.37 & -0.08 \\
\hline PC-F3 & 0.62 & $25.0 \mathrm{~L}$ & $51.9 \mathrm{~L}$ & $46.8 \mathrm{H}$ & 0 & 1.65 & 2.30 & 1.08 & 2.54 & 1.01 & -1.46 \\
\hline VA2 & 1.38 & $92.3 \mathrm{~L}$ & $92.3 \mathrm{~L}$ & 0 & 0 & 0.98 & 0.96 & 1.24 & 2.83 & 0.60 & -1.92 \\
\hline VA3 & 1.72 & $27.9 \mathrm{~L}$ & $73.6 \mathrm{~L}$ & $100 \mathrm{U}$ & $83.2 \mathrm{H}$ & 1.84 & 2.69 & 2.58 & 5.24 & -0.05 & -2.62 \\
\hline VA4 & 2.31 & $100 \mathrm{U}$ & $100 \mathrm{U}$ & $100 \mathrm{U}$ & 0 & 0.91 & 0.82 & 1.23 & 2.81 & -0.42 & -1.77 \\
\hline VZ0 & 8.33 & $100 \mathrm{U}$ & $100 \mathrm{U}$ & $100 \mathrm{U}$ & 0 & 1.59 & 2.17 & 1.22 & 2.80 & 1.78 & -1.62 \\
\hline VZ1 & 3.74 & $100 \mathrm{U}$ & $100 \mathrm{U}$ & $100 \mathrm{U}$ & 0 & 0.54 & 0.09 & 0.57 & 1.63 & -0.68 & -1.38 \\
\hline VZ2 & 2.35 & $5.4 \mathrm{~L} / 64.7 \mathrm{H}$ & $5.4 \mathrm{~L} / 64.7 \mathrm{H}$ & $20.8 \mathrm{~L}$ & 0 & 0.96 & 0.92 & 1.27 & 2.89 & -1.81 & -1.92 \\
\hline VZ3 & 1.69 & $31.7 \mathrm{~L}$ & $73.3 \mathrm{~L}$ & $100 \mathrm{U}$ & 0 & 0.92 & 0.84 & 1.20 & 2.76 & -4.01 & -1.77 \\
\hline VZ4 & 1.89 & $100 \mathrm{U}$ & $100 \mathrm{U}$ & $100 \mathrm{U}$ & 0 & 0.54 & 0.08 & 0.61 & 1.70 & -0.49 & -1.38 \\
\hline \multicolumn{12}{|c|}{$\delta$ meson models } \\
\hline$\overline{\mathrm{DD}}-\mathrm{ME} \delta$ & -0.26 & 0 & 0 & 0 & 0 & 0.44 & -0.13 & -0.41 & -0.14 & 1.94 & -0.31 \\
\hline $\mathrm{D} D H \delta$ & 0.25 & 0 & 0 & 0 & 0 & -0.93 & -2.86 & -0.55 & -0.39 & -2.44 & -0.08 \\
\hline GDFM & 2.35 & $100 \mathrm{U}$ & $100 \mathrm{U}$ & $90.4 \mathrm{~L}$ & $11.8 \mathrm{~L}$ & 0.61 & 0.22 & -0.05 & 0.52 & -0.44 & -0.31 \\
\hline $\mathrm{N} L \delta$ & 0.25 & $2.2 \mathrm{~L}$ & $2.2 \mathrm{~L}$ & 0 & $68.8 \mathrm{~L}$ & 0.12 & -0.76 & 0.70 & 1.86 & 2.89 & -1.62 \\
\hline NL3 $\delta$ & 1.00 & $100 \mathrm{U}$ & $100 \mathrm{U}$ & $86.4 \mathrm{~L}$ & 71.1L & 1.53 & 2.07 & 1.78 & 3.80 & 1.69 & -1.92 \\
\hline
\end{tabular}


[1] C. F. von Weizsäcker, Z. Phys. 96, 431 (1935).

[2] T. H. R. Skyrme, Philos. Mag. 1, 1043 (1956).

[3] D. Vautherin and D. M. Brink, Phys. Rev. C 5, 626 (1972).

[4] J. D. Walecka, Ann. Phys. 83, 491 (1974).

[5] M. Dutra, O. Lourenço, J. S. Sá Martins, A. Delfino, J. R. Stone, and P. D. Stevenson, Phys. Rev. C 85, 035201 (2012).

[6] J. M. Pearson, N. Chamel, and S. Goriely, Phys. Rev. C 82, 037301 (2010).

[7] E. Lipparini and S. Stringari, Phys. Rep. 175, 103 (1989).

[8] J. P. Blaizot and B. Grammaticos, Nucl. Phys. A 265, 315 (1976).

[9] S. Shlomo, V. M. Kolomietz, and G. Colo, Eur. Phys. J. A 30, 23 (2006); T. Sil, S. Shlomo, B. K. Agrawal, and P. G. Reinhard, Phys. Rev. C 73, 034316 (2006); B. K. Agrawal, S. Shlomo, and V. Kim Au, ibid. 68, 031304 (2003).

[10] J. R. Stone, N. J. Stone, and S. A. Moszkowski, Phys. Rev. C 89, 044316 (2014).

[11] E. Khan, J. Margueron, and I. Vidaña, Phys. Rev. Lett. 109, 092501 (2012).

[12] E. Khan and J. Margueron, Phys. Rev. C 88, 034319 (2013).

[13] M. B. Tsang, Y. Zhang, P. Danielewicz, M. Famiano, Z. Li, W. G. Lynch, and A. W. Steiner, Phys. Rev. Lett. 102, 122701 (2009); Z. Kohley et al., Phys. Rev. C 83, 044601 (2011).

[14] A. Klimkiewicz et al., Phys. Rev. C 76, 051603(R) (2007); O. Wieland et al., Phys. Rev. Lett. 102, 092502 (2009).

[15] A. Carbone, G. Colo, A. Bracco, L.-G. Cao, P. F. Bortignon, F. Camera, and O. Wieland, Phys. Rev. C 81, 041301 (2010).

[16] P. Danielewicz and J. Lee, AIP Conf. Proc. 1423, 29 (2012).

[17] C. J. Horowitz and J. Piekarewicz, Phys. Rev. Lett. 86, 5647 (2001).

[18] M. B. Tsang, J. R. Stone, F. Camera, P. Danielewicz, S. Gandolfi, K. Hebeler, C. J. Horowitz, J. Lee, W. G. Lynch, Z. Kohley, R. Lemmon, P. Moller, T. Murakami, S. Riordan, X. Roca-Maza, F. Sammarruca, A. W. Steiner, I. Vidaña, and S. J. Yennello, Phys. Rev. C 86, 015803 (2012).

[19] A. Krugmann, D. Martin, P. von Neumann-Cosel, N. Pietralla, and A. Tamii for the E350 Collaboration, EPJ Web Conf. 66, 02060 (2014).

[20] C. Providência, S. S. Avancini, R. Cavagnoli, S. Chiacchiera, C. Ducoin, F. Grill, J. Margueron, D. P. Menezes, A. Rabhi, and I. Vidaña, Eur. Phys. J. A 50, 44 (2014).

[21] J. Piekarewicz, Phys. Rev. C 66, 034305 (2002).

[22] J. Piekarewicz, Phys. Rev. C 69, 041301 (2004).

[23] C. Ducoin, J. Margueron, C. Providência, and I. Vidaña, Phys. Rev. C 83, 045810 (2011).

[24] J. M. Lattimer and Y. Lim, Astrophys. J. 771, 51 (2013).

[25] B. M. Santos, M. Dutra, O. Lourenço, and A. Delfino, Phys. Rev. C 90, 035203 (2014).

[26] L. W. Chen, C. M. Ko, and B.-A. Li, Phys. Rev. Lett. 94, 032701 (2005); Phys. Rev. C 72, 064309 (2005); B.-A. Li, L. W. Chen, and C. M. Ko, Phys. Rep. 464, 113 (2008).

[27] M. Centelles, X. Roca-Maza, X. Viñas, and M. Warda, Phys. Rev. Lett. 102, 122502 (2009).

[28] T. Li et al., Phys. Rev. Lett. 99, 162503 (2007).

[29] B. D. Lackey, M. Nayyar, and B. J. Owen, Phys. Rev. D 73, 024021 (2006).

[30] J. Boguta and A. R. Bodmer, Nucl. Phys. A 292, 413 (1977).

[31] S. Typel and H. H. Wolter, Nucl. Phys. A 656, 331 (1999).

[32] P. Gögelein, E. N. E. van Dalen, C. Fuchs, and H. Müther, Phys. Rev. C 77, 025802 (2008).
[33] S. S. Avancini, L. Brito, Ph. Chomaz, D. P. Menezes, and C. Providência, Phys. Rev. C 74, 024317 (2006).

[34] B. A. Nikolaus, T. Hoch, and D. G. Madland, Phys. Rev. C 46, 1757 (1992).

[35] G. Gelmini and B. Ritzi, Phys. Lett. B 357, 431 (1995); A. Delfino, M. Malheiro, and T. Frederico, Braz. J. Phys. 31, 518 (2001).

[36] O. Lourenço, R. L. P. G. Amaral, M. Dutra, and A. Delfino, Phys. Rev. C 80, 045206 (2009); O. Lourenço, M. Dutra, A. Delfino, and R. L. P. G. Amaral, Int. J. Mod. Phys. E 16, 3037 (2007).

[37] I. Vidaña, C. Providência, A. Polls, and A. Rios, Phys. Rev. C 80, 045806 (2009).

[38] J. B. Silva, O. Lourenço, A. Delfino, J. S. Sá Martins, and M. Dutra, Phys. Lett. B 664, 246 (2008); J. B. Elliott, P. T. Lake, L. G. Moretto, and L. Phair, Phys. Rev. C 87, 054622 (2013).

[39] D. H. Youngblood, H. L. Clark, and Y.-W. Lui, Phys. Rev. Lett. 82, 691 (1999).

[40] M. Farine, J. M. Pearson, and F. Tondeur, Nucl. Phys. A 615, 135 (1997).

[41] P. Danielewicz, R. Lacey, and W. G. Lynch, Science 298, 1592 (2002).

[42] W. G. Lynch, M. B. Tsang, Y. Zhang, P. Danielewicz, M. Famiano, Z. Li, and A. W. Steiner, Prog. Part. Nucl. Phys. 62, 427 (2009).

[43] J. R. Stone and P. G. Reinhard, Prog. Part. Nucl. Phys. 58, 587 (2007).

[44] L. W. Chen, C. M. Ko, Bao-An Li, and J. Xu, Phys. Rev. C 82, 024321 (2010).

[45] P. Danielewicz, Nucl. Phys. A 727, 233 (2003).

[46] J. Piekarewicz, Phys. Rev. C 76, 064310 (2007).

[47] A. Gezerlis and J. Carlson, Phys. Rev. C 77, 032801 (2008).

[48] A. Schwenk and C. J. Pethick, Phys. Rev. Lett. 95, 160401 (2005).

[49] J. Carlson, S.-Y. Chang, V. R. Pandharipande, and K. E. Schmidt, Phys. Rev. Lett. 91, 050401 (2003).

[50] E. Epelbaum, H. Krebs, D. Lee, and U.-G. Meissner, Eur. Phys. J. A 40, 199 (2009).

[51] M. Bartenstein, A. Altmeyer, S. Riedl, S. Jochim, C. Chin, J. H. Denschlag, and R. Grimm, Phys. Rev. Lett. 92, 120401 (2004).

[52] J. Kinast, A. Turlapov, J. E. Thomas, Q. Chen, J. Stajic, and K. Levin, Science 307, 1296 (2005).

[53] J. T. Stewart, J. P. Gaebler, C. A. Regal, and D. S. Jin, Phys. Rev. Lett. 97, 220406 (2006).

[54] L. Luo and J. E. Thomas, J. Low Temp. Phys. 154, 1 (2009).

[55] L. Brito, Ph. Chomaz, D. P. Menezes, and C. Providência, Phys. Rev. C 76, 044316 (2007).

[56] P. Möller, W. D. Meyers, W. J. Swiatecki, and J. Treiner, At. Data Nucl. Data Tables 39, 225 (1988).

[57] B. A. Brown, Phys. Rev. Lett. 85, 5296 (2000).

[58] D. H. Youngblood, Y. W. Lui, H. L. Clark, B. John, Y. Tokimoto, and X. Chen, Phys. Rev. C 69, 034315 (2004).

[59] M. Uchida et al., Phys. Rev. C 69, 051301 (2004).

[60] M. Uchida et al., Phys. Lett. B 557, 12 (2003).

[61] T. Li et al., Phys. Rev. C 81, 034309 (2010).

[62] A. W. Steiner, J. M. Lattimer, and E. F. Brown, Astrophys. J. Lett. 765, L5 (2013).

[63] J. M. Lattimer and A. W. Steiner, Astrophys. J. 784, 123 (2014).

[64] S. Guillot, M. Servillat, N. A. Webb, and R. E. Rutledge, Astrophys. J. 772, 7 (2013). 
[65] B.-A. Li and X. Han, Phys. Lett. B 727, 276 (2013).

[66] B.-A. Li et al., J. Phys.: Conf. Ser. 312, 042006 (2011).

[67] B. K. Agrawal, Phys. Rev. C 81, 034323 (2010).

[68] N. K. Glendenning, Compact Stars: Nuclear Physics, Particle Physics, and General Relativity, 2nd ed. (Springer, New York, 2000).

[69] P. B. Demorest et al., Nature (London) 467, 1081 (2010).

[70] J. Antoniadis et al., Science 340, 1233232 (2013).

[71] S. Weissenborn, D. Chatterjee, and J. Schaffner-Bielich, Phys. Rev. C 85, 065802 (2012); Nucl. Phys. A 881, 62 (2012).

[72] R. Cavagnoli, D. P. Menezes, and C. Providência, Phys. Rev. C 84, 065810 (2011); C. Providência and A. Rabhi, ibid. 87, 055801 (2013).

[73] L. L. Lopes and D. P. Menezes, Phys. Rev. C 89, 025805 (2014).

[74] P. A. M. Guichon, Phys. Lett. B 200, 235 (1988); K. Saito and A. W. Thomas, ibid. 327, 9 (1994); J. R. Stone, P. A. Guichon et al., Nucl. Phys. A 792, 341 (2007); P. K. Panda, D. P. Menezes, and C. Providência, Phys. Rev. C 89, 045803 (2014).

[75] P. D. Stevenson, P. M. Goddard, J. R. Stone, and M. Dutra, AIP Conf. Proc. 1529, 262 (2013).

[76] S. Haddad, Europhys. Lett. 48, 505 (1999).

[77] P.-G. Reinhard, Rep. Prog. Phys. 52, 439 (1989).

[78] Internal discussion notes, ORNL, University of Erlangen, University of Frankfurt, University of Tennessee Knoxville, University of Oxford, Vanderbilt University, and University of Warsaw (private communication).

[79] A. A. Dadi, Phys. Rev. C 82, 025203 (2010).

[80] M. Rufa, P.-G. Reinhard, J. A. Maruhn, W. Greiner, and M. R. Strayer, Phys. Rev. C 38, 390 (1988).

[81] N. K. Glendenning, Phys. Lett. B 114, 392 (1982).

[82] N. K. Glendenning and S. A. Moszkowski, Phys. Rev. Lett. 67, 2414 (1991).

[83] S. K. Ghosh, S. C. Phatak, and P. K. Sahu, Z. Phys. A 352, 457 (1995).

[84] J. Piekarewicz and M. Centelles, Phys. Rev. C 79, 054311 (2009).

[85] H. Müller and B. D. Serot, Nucl. Phys. A 606, 508 (1996).

[86] M. Bender, K. Rutz, P. G. Reinhard, J. A. Maruhn, and W. Greiner, Phys. Rev. C 60, 034304 (1999).

[87] B. Liu, V. Greco, V. Baran, M. Colonna, and M. DiToro, Phys. Rev. C 65, 045201 (2002).

[88] M. Centelles, M. D. Estal, and X. Viñas, Nucl. Phys. A 635, 193 (1998).

[89] G. A. Lalazissis, J. König, and P. Ring, Phys. Rev. C 55, 540 (1997).

[90] G. A. Lalazissis et al., Phys. Lett. B 671, 36 (2009).

[91] B. Nerlo-Pomoroska and J. Sykut, Int. J. Mod. Phys. E 13, 75 (2004).

[92] B. D. Serot and J. D. Walecka, Int. J. Mod. Phys. E 6, 515 (1997).

[93] B. D. Serot, Rep. Prog. Phys. 55, 1855 (1992).

[94] K. C. Chung et al., Eur. Phys. J. A 9, 453 (2000).

[95] M. Rashdan, Phys. Rev. C 63, 044303 (2001).
[96] P.-G. Reinhard, Z. Phys. A 329, 257 (1988).

[97] A. Sulaksono, T. Mart, and C. Bahri, Phys. Rev. C 71, 034312 (2005).

[98] R. J. Furnstahl, B. D. Serot, and H. B. Tang, Nucl. Phys. A 615, 441 (1997).

[99] S. Gmuca, Z. Phys. A 342, 387 (1992).

[100] M. Centelles et al., Nucl. Phys. A 537, 486 (1992).

[101] S. Gmuca, Nucl. Phys. A 547, 447 (1992).

[102] J. K. Bunta and S. Gmuca, Phys. Rev. C 68, 054318 (2003).

[103] H. Müller and B. D. Serot, Phys. Rev. C 52, 2072 (1995).

[104] F. J. Fattoyev and J. Piekarewicz, Phys. Rev. C 82, 025805 (2010).

[105] M. M. Sharma, A. R. Farhan, and S. Mythili, Phys. Rev. C 61, 054306 (2000).

[106] W. Long, J. Meng, N. VanGiai, and S. G. Zhou, Phys. Rev. C 69, 034319 (2004).

[107] Y. Sugahara and H. Toki, Nucl. Phys. A 579, 557 (1994).

[108] H. Toki et al., Nucl. Phys. A 588, 357 (1995).

[109] S. K. Dhiman, R. Kumar, and B. K. Agrawal, Phys. Rev. C 76, 045801 (2007).

[110] B.-J. Cai and L.-W. Chen, Phys. Rev. C 85, 024302 (2012).

[111] B. G. Todd-Rutel and J. Piekarewicz, Phys. Rev. Lett. 95, 122501 (2005).

[112] J. Piekarewicz and S. P. Weppner, Nucl. Phys. A 778, 10 (2006).

[113] R. Kumar, B. K. Agrawal, and S. K. Dhiman, Phys. Rev. C 74, 034323 (2006).

[114] A. Sulaksono and T. Mart, Phys. Rev. C 74, 045806 (2006).

[115] F. J. Fattoyev, C. J. Horowitz, J. Piekarewicz, and G. Shen, Phys. Rev. C 82, 055803 (2010).

[116] C. J. Horowitz and J. Piekarewicz, Phys. Rev. C 66, 055803 (2002).

[117] M. M. Haidari and M. M. Sharma, Nucl. Phys. A 803, 159 (2008).

[118] M. M. Sharma, Phys. Lett. B 666, 140 (2008).

[119] M. DelEstal, M. Centelles, X. Viñas, and S. K. Patra, Phys. Rev. C 63, 024314 (2001).

[120] S. Typel, Phys. Rev. C 71, 064301 (2005).

[121] T. Klähn et al., Phys. Rev. C 74, 035802 (2006).

[122] T. Nikšić, D. Vretenar, P. Finelli, and P. Ring, Phys. Rev. C 66, 024306 (2002).

[123] G. A. Lalazissis, T. Nikšić, D. Vretenar, and P. Ring, Phys. Rev. C 71, 024312 (2005).

[124] S. Typel, G. Röpke, T. Klähn, D. Blaschke, and H. H. Wolter, Phys. Rev. C 81, 015803 (2010).

[125] J. J. Rusnak and R. J. Furnstahl, Nucl. Phys. A 627, 495 (1997).

[126] T. Bürvenich, D. G. Madland, J. A. Maruhn, and P.-G. Reinhard, Phys. Rev. C 65, 044308 (2002).

[127] X. Roca-Maza, X. Viñas, M. Centelles, P. Ring, and P. Schuck, Phys. Rev. C 84, 054309 (2011).

[128] T. Gaitanos, M. Di Toro, S. Typel, V. Baran, C. Fuchs, V. Greco, and H. H. Wolter, Nucl. Phys. A 732, 24 (2004).

[129] D. P. Menezes and C. Providência, Phys. Rev. C 70, 058801 (2004). 